\title{
Radiative stellar feedback in galaxy formation: Methods and physics
}

\author{
Philip F. Hopkins ${ }^{\oplus},{ }^{1 \star}$ Michael Y. Grudić ${ }^{\oplus},{ }^{1}$ Andrew Wetzel ${ }^{\oplus},{ }^{2}$ Dušan Kereš, ${ }^{3}$ \\ Claude-André Faucher-Giguère ${ }^{\oplus},{ }^{4}$ Xiangcheng $\mathrm{Ma}^{\oplus},{ }^{5}$ Norman Murray ${ }^{6}$ \\ and Nathan Butcher ${ }^{3}$ \\ ${ }^{1}$ TAPIR, Mailcode 350-17, California Institute of Technology, Pasadena, CA 91125, USA \\ ${ }^{2}$ Department of Physics, University of California, Davis, CA 95616, USA \\ ${ }^{3}$ Department of Physics, Center for Astrophysics and Space Science, University of California at San Diego, 9500 Gilman Drive, La Jolla, CA 92093, USA \\ ${ }^{4}$ Department of Physics and Astronomy and CIERA, Northwestern University, 2145 Sheridan Road, Evanston, IL 60208, USA \\ ${ }^{5}$ Department of Astronomy and Theoretical Astrophysics Center, University of California Berkeley, Berkeley, CA 94720, USA \\ ${ }^{6}$ Canadian Institute for Theoretical Astrophysics, 60 St. George Street, University of Toronto, ON M5S 3H8, Canada
}

Accepted 2019 November 4. Received 2019 October 30; in original form 2018 November 26

\begin{abstract}
Radiative feedback (RFB) from stars plays a key role in galaxies, but remains poorly understood. We explore this using high-resolution, multifrequency radiation-hydrodynamics (RHD) simulations from the Feedback In Realistic Environments (FIRE) project. We study ultrafaint dwarf through Milky Way mass scales, including $\mathrm{H}+$ He photoionization; photoelectric, Lyman Werner, Compton, and dust heating; and single+multiple scattering radiation pressure (RP). We compare distinct numerical algorithms: ray-based LEBRON (exact when optically thin) and moments-based M1 (exact when optically thick). The most important RFB channels on galaxy scales are photoionization heating and single-scattering RP: in all galaxies, most ionizing/far-UV luminosity ( $\sim 1 / 2$ of lifetime-integrated bolometric) is absorbed. In dwarfs, the most important effect is photoionization heating from the UV background suppressing accretion. In MW-mass galaxies, metagalactic backgrounds have negligible effects; but local photoionization and single-scattering RP contribute to regulating the galactic star formation efficiency and lowering central densities. Without some RFB (or other 'rapid' FB), resolved GMCs convert too-efficiently into stars, making galaxies dominated by hyperdense, bound star clusters. This makes star formation more violent and 'bursty' when SNe explode in these hyperclustered objects: thus, including RFB 'smoothes' SFHs. These conclusions are robust to RHD methods, but M1 produces somewhat stronger effects. Like in previous FIRE simulations, IR multiple-scattering is rare (negligible in dwarfs, $\sim 10$ per cent of RP in massive galaxies): absorption occurs primarily in 'normal' GMCs with $A_{V} \sim 1$.
\end{abstract}

Key words: stars: formation-galaxies: active-galaxies: evolution-galaxies: formationcosmology: theory.

\section{INTRODUCTION}

Stars are not passive gravitational 'sinks.' Rather, once formed, the radiation, winds, and explosions of (especially massive) stars dramatically alter subsequent star and even galaxy formation. If these 'feedback' processes are not included, gas in galaxies or giant molecular clouds (GMCs) collapses, fragments, and turns entirely into stars within a couple free-fall times (Bournaud et al. 2010; Dobbs, Burkert \& Pringle 2011; Harper-Clark \& Murray 2011; Hopkins, Quataert \& Murray 2011; Tasker 2011), eventually turning most of the baryons in the Universe into stars (Katz,

^E-mail: phopkins@caltech.edu
Weinberg \& Hernquist 1996; Somerville \& Primack 1999; Cole et al. 2000; Springel \& Hernquist 2003b; Kereš et al. 2009; FaucherGiguère, Kereš \& Ma 2011). In reality, observed GMCs appear to convert just a few percent of their mass into stars before being disrupted via feedback (Zuckerman \& Evans 1974; Williams \& McKee 1997; Evans 1999; Evans et al. 2009), only a percent or so of the gas on a galaxy scale turns into stars per (galactic) free-fall time (Kennicutt 1998), and only a few percent of the baryons remain in galaxies (Conroy, Wechsler \& Kravtsov 2006; Behroozi, Conroy \& Wechsler 2010; Moster et al. 2010) while the rest are expelled into the circumgalactic and intergalactic medium in outflows (Martin 1999; Heckman et al. 2000; Pettini et al. 2003; Songaila 2005; Sato et al. 2009; Martin et al. 2010; Steidel et al. 2010). 
'Feedback' is an umbrella term incorporating many processes including protostellar jets, photoheating, stellar mass-loss, radiation pressure, supernovae (Types Ia \& II), cosmic ray acceleration, and more (e.g. Evans et al. 2009; Lopez et al. 2011). Until recently, in simulations of galaxies, the ISM on $\sim \mathrm{kpc}$ scales, or large GMC complexes $\left(\gtrsim 10^{6} \mathrm{M}_{\odot}\right)$, it was not possible to model these processes directly and so simplified 'sub-grid' prescriptions were used to model the ultimate effects of feedback (e.g. directly launching winds from clouds or galaxies); however, in recent years simulations have begun to directly resolve the multiphase structure in the ISM and therefore have attempted to treat these feedback channels explicitly (e.g. Hopkins et al. 2011; Tasker 2011; Hopkins, Quataert \& Murray 2012b; Wise et al. 2012; Agertz et al. 2013; Kannan et al. 2014a; Roškar et al. 2014). For one example, studies of galaxy formation and star cluster formation from the Feedback In Realistic Environments (FIRE) ${ }^{1}$ project Hopkins et al. (2014) explicitly treat the multiphase structure of gas from $\sim 10-10^{10} \mathrm{~K}$ with star formation only in self-gravitating, self-shielding dense gas and resolution reaching $\sim 30 \mathrm{M}_{\odot}$ in cosmological galaxyformation simulations (Ma et al. 2018; Wheeler et al. 2019) or $\sim 0.01 \mathrm{M}_{\odot}$ in massive GMC complex/star cluster simulations (Grudić et al. 2018b, a). At this resolution, the simulations attempt to explicitly follow different stellar feedback channels with the spectral, energy, momentum, mass, and metal fluxes in stellar massloss, $\mathrm{SNe}$, and radiation taken directly from stellar evolution models.

On these scales, it is widely agreed that radiation from stars 'radiative feedback' - plays a role in galaxy and star formation. Photoionization by starlight is necessary to sustain H II regions (which can in turn expand and destroy GMCs), the warm interstellar medium (WIM), and the metagalactic UV background (e.g. Tielens 2005), which in turn can suppress dwarf galaxy formation (Barkana \& Loeb 2001). Photoelectric heating is critical to the structure of the cold/warm neutral medium (C/WNM; Wolfire et al. 1995). Radiation pressure inputs a single-scattering momentum flux into the ISM of $\dot{p} \sim L / c$, comparable (at least initially) to the momentum injection from stellar winds and $\mathrm{SNe}$ (Leitherer et al. 1999), and in very dense regions which are optically thick to infrared (IR) radiation, multiple-scattering can increase this by a factor up to a maximum of $\sim 1+\tau_{\text {IR }}$ (the IR optical depth; Murray, Quataert \& Thompson 2005). At this point, essentially all observational and theoretical studies agree that radiation has an important impact on star formation at the scales of individual stars (Rosen et al. 2016) and cores (Bate, Tricco \& Price 2014; Guszejnov, Hopkins \& Grudić 2018), clumps (Hopkins \& Grudic 2018; Grudić et al. 2018b), GMCs (Murray, Quataert \& Thompson 2010; HarperClark \& Murray 2011; Hopkins et al. 2012b; Howard, Pudritz \& Harris 2016), and clusters (Lopez et al. 2011; Colín, VázquezSemadeni \& Gómez 2013; Grudić et al. 2018a, 2019). However, controversy about the ultimate impact of these processes for bulk galaxy properties (e.g. SFRs, galaxy masses) has abounded, much of it centred on questions of the numerical methods used to treat the radiation (Krumholz \& Thompson 2012; Jiang, Davis \& Stone 2013; Davis et al. 2014; Sales et al. 2014; Tsang \& Milosavljević 2015). Most (though not all) of this work has focused on idealized studies, which (while critical for understanding the microphysics involved; see e.g. Takeuchi, Ohsuga \& Mineshige 2014; Skinner \& Ostriker 2015; Raskutti, Ostriker \& Skinner 2016; Zhang \& Davis 2017;

\footnotetext{
${ }^{1}$ See the FIRE project website: http://fire.northwestern.edu. For additional movies and images of FIRE simulations, see: http://www.tapir.caltech.ed $\mathrm{u} /$ phopkins/Site/animations/.
}

Zhang et al. 2018) do not clearly map to consequences for global galaxy properties; even galactic studies have largely been focused on high-resolution simulations of idealized, non-cosmological, single galaxies (Bieri et al. 2017; Costa et al. 2018a; Emerick, Bryan \& Mac Low 2018; Kannan et al. 2019). But this misses potentially key regimes in galaxy mass and/or redshift, where the physics may change in important ways. On the other hand, obviously, large-scale simulations must capture at least some of the key scales (e.g. the existence of GMCs and phase structure in the ISM around massive stars) needed to compute the effects of radiative feedback, or else they cannot predict its consequences.

In a companion paper Hopkins et al. (2018b, hereafter Paper I), we presented an updated version of the FIRE code, 'FIRE-2,' and considered a wide range of numerical effects (e.g. resolution, hydrodynamic solvers), as well as the effects of various 'non-feedback' physics (e.g. cooling, star formation) on galaxy formation, in fully-cosmological simulations which follow the physics described above. These can begin to explore these critical questions for feedback. A follow-up paper (Hopkins et al. 2018a, hereafter Paper II) specifically explored the importance of numerical methods and resolution-scale physics in coupling mechanical feedback from stars (e.g. SNe and stellar mass-loss). This paper therefore continues these studies by exploring the role of radiative feedback in galaxy formation.

Specifically, we will use these simulations to explore the importance of both numerical methods and different aspects of radiative feedback physics, for global galaxy properties. This includes e.g. star formation rates and histories (SFRs/SFHs); stellar masses; metallicities/abundances; stellar, baryonic, and dark matter mass profiles and content within the halo; circular velocity profiles; visual morphologies; and the distribution of gas in different phases. In order to explore how the effects depend on mass, we will consider a range of galaxy masses from ultrafaint dwarfs to Milky Way and Andromeda-mass systems - however, we will exclusively focus on radiative feedback from stars in this article, meaning we will not consider AGN feedback, so we cannot consider galaxies much more massive than $\sim L_{*}$. We will survey a wide range of different radiative feedback channels (e.g. radiation pressure in UV versus IR, H, and He photoionization, photoelectric heating, Compton heating, etc.) as described in Section 2 below. Finally, because the RHD methods which can be solved efficiently in large cosmological simulations are necessarily approximate, we will compare all of the above using two fundamentally distinct numerical methods for approximating and discretizing the RHD equations.

In Section 2, we briefly review the various processes collectively referred to as 'radiative feedback' explored here. In Section 3 we discuss our simulation methods, and in Section 4 we systematically explore the effects of each of these radiative feedback processes in our galaxy formation simulations. We summarize the major effects of radiative feedback as a function of galaxy mass (Section 4.1) and specifically discuss degeneracies with other 'early' feedback processes (Section 4.2) before systematically exploring each radiative feedback process in turn (Section 4.3), as well as discussing where and how photons actually couple in the simulations (Section 4.4) and the effects of numerical methods (Section 4.5). We summarize and conclude in Section 5.

\section{WHAT IS RADIATIVE FEEDBACK?}

'Radiative feedback' is itself an umbrella term referring to a huge range of processes. We attempt to enumerate some of these here, because although we will explore many of them, it is impossible to 
be exhaustive and we wish to be clear about which processes we do not address in this paper. We will focus exclusively on galaxy scales.

Broadly speaking, 'radiative feedback' can be divided into three categories: 'radiative heating,' 'indirect feedback (ionization/dissociation effects),' and 'radiation pressure.'

\subsection{Radiative heating}

Here we consider processes that directly transfer thermal energy to gas, probably the best-studied form of radiative feedback on large scales. This takes many forms, including:

(i) Photoionization heating from local sources: Photoionization heating (to $\sim 10^{4}-10^{5} \mathrm{~K}$ ) by ionizing photons around massive stars (with flux dominated by nearby stars in a galaxy/cluster) is critical to the WIM and HII regions. This will be considered throughout this paper.

(ii) Photoionization heating from 'collective' effects: Although ultimately the same sources, we distinguish the collective photoionization heating by many galaxies (via 'escaped' photons) in the form of the metagalactic UV background (UVB), critical for the phase structure of the circumgalactic medium (CGM) and intergalactic medium (IGM), Ly $\alpha$ forest, and believed to suppress $\mathrm{SF}$ in small dwarf galaxies. This will also be considered throughout, with the approximation of a (spatially) uniform background plus local self-shielding.

(iii) Photoelectric heating: Local UV (non-ionizing) luminosity absorbed by dust generates photoelectric heating (photoadsorption/desorption can also be included), important for thermal balance/chemistry in the WNM/CNM $(T \sim 100-8000 \mathrm{~K})$. It has been controversial whether this has any dynamical effects on SF on galaxy scales; it is included in our default RHD treatment, but we will explore removing it as well.

(iv) Compton heating: Hard photons can Compton-heat diffuse gas to high temperatures; this is potentially important around AGN ( $T_{\text {Compton }} \gtrsim 10^{7} \mathrm{~K}$ ). From stars alone (where it depends on the much-less-luminous X-ray binary [XRB] population) it is not likely to be dominant (Sazonov, Ostriker \& Sunyaev 2004; Oppenheimer et al. 2018), so we will only briefly consider it (in a limited subset of tests with our 'extended' RHD network) for completeness.

(v) Thermal dust/collisional heating: At high densities $(n \gg$ $\left.10^{6} \mathrm{~cm}^{-3}\right)$ and low temperatures $(T \ll 100 \mathrm{~K})$ dust is collisionally tightly coupled to gas and thermal heating of dust by IR radiation can transfer heat to gas. This is critical to protostellar accretion and may explain the Universality of the IMF (Bate et al. 2014; Offner et al. 2014; Guszejnov, Krumholz \& Hopkins 2016; Guszejnov, Hopkins \& Krumholz 2017; Guszejnov, Hopkins \& Ma 2017), but is negligible at the much lower density scales (and mass scales $\gg 0.1 \mathrm{M}_{\odot}$ ) resolved here. We will only briefly consider it for completeness (although we may be effectively modelling it implicitly, by assuming an IMF).

\subsection{Indirect radiative feedback (ionization/dissociation effects)}

This refers to processes which alter subsequent gas cooling rates or star formation via, e.g. ionization or dissociation.

(i) $\mathbf{H} \&$ He ionization: Photoionization of $\mathrm{H}$ and $\mathrm{He}$, as described above, not only directly contributes a heating term but also alters the cooling rates non-linearly (changing e.g. the number of free electrons, recombination rates, etc.). This is always included in our simulations alongside the self-consistent ionization calculations (see Paper I for details). (ii) Metal ionization: In the CGM/IGM, the UVB partially ionizes metals, which alters their line cooling properties. But local, hard sources can (under special circumstances) dominate over the collective UVB and 'overionize' those metals, further suppressing line cooling (lowering the cooling rates), although this likely requires non-equilibrium chemistry to treat properly. Whenever we include the effects of the UVB, the effect on metal ionization of the UVB is also self-consistently included (in the tabulated cooling rates for metal-line cooling). However, we will not explore the effects of non-equilibrium overionization from local sources in this paper (see Richings \& Schaye 2016; Oppenheimer et al. 2018, for more discussion).

(iii) Lyman-Werner feedback: Dissociation of $\mathrm{H}_{2}$ by LymanWerner radiation can suppress molecular-hydrogen cooling in lowtemperature gas, potentially important in extremely metal-poor firststar environments (by $Z \gtrsim 10^{-5}-10^{-3} Z_{\odot}$, metal-line and dust cooling dominates $\mathrm{H}_{2}$ cooling at low temperatures). Because we do not follow explicit molecular chemistry, and our feedback and yield physics does not include any separate model for Pop III stars, we will not consider this in detail in this paper, although we will briefly discuss approximate treatments (but see e.g. Wise \& Abel 2008; Wise et al. 2012).

\subsection{Radiation pressure}

Here we consider processes that transfer momentum or kinetic energy to gas.

(i) Single scattering ('Direct'): Photons carry momentum $h \mathrm{v} / c$; if gas or dust absorption 'destroys' the photon (no re-emission, or isotropic re-emission at longer wavelengths), then this is transferred to dust and gas as a momentum flux $\sim L_{\text {absorbed }} / c$. This can be comparable to or larger than gravitational forces in $\mathrm{H}$ II regions, massive GMCs, and even galaxy scales. We will consider this throughout for all photons at frequencies above infrared.

(ii) Multiple scattering (Continuum): If photons are repeatedly scattered (e.g. Thompson scattering) or re-emitted at wavelengths with similar opacities (e.g. IR re-emission and re-absorption) the repeated scattering can transfer additional momentum to gas, up to a maximum momentum flux $\sim \tau L / c$. Our default RHD treatment accounts for this for infrared photons, in the grey approximation (frequency-independent IR opacity).

(iii) Line driving: This is the same concept as multiple-scattering above, but we distinguish multiple scattering in resonance lines because it requires line transfer, and the 'escape' by scattering out-of-resonance is distinct. This is believed to be critical for mass-loss from massive stars, and certain types of accretion-disc winds in AGN, but on galactic scales is not believed to be critical except, potentially, for resonant Ly $\alpha$ line scattering. However properly treating resonant Ly $\alpha$ scattering is physically challenging (usually requiring custom algorithms even to post-process results; Faucher-Giguère et al. 2010; Smith et al. 2019) and extremely computationally demanding, so we will not consider it here (but see Smith, Bromm \& Loeb 2017; Kimm et al. 2018; Smith et al. 2018 for recent studies)

\section{SIMULATION METHODS}

\subsection{Overview}

The simulations in this paper were run as part of the FIRE; specifically using the 'FIRE-2' version of the code from Paper I. 
In this paper we will systematically vary the treatment of radiative feedback, but all other simulation physics, initial conditions, and numerical parameters are held fixed. Paper I contains all details of these aspects of the method, so we only briefly summarize them here.

The simulations are run using GIZMO, a radiationmagnetohydrodynamics code ${ }^{2}$ in its Lagrangian Godunov 'meshless finite mass' (MFM) mode (for extensive test problems see Hopkins 2015; Hopkins \& Raives 2016; Hopkins 2016, 2017). They are fully cosmological 'zoom-in' simulations which embed a high-resolution Lagrangian region, that will surround a single $z=$ 0 galaxy, in a large cosmological box initialized at $z \sim 100$. Gravity is treated with adaptive softenings so hydrodynamic and force softenings are always matched, with no artificial minimum enforced. Gas cooling is followed over $T=10-10^{10} \mathrm{~K}$ including free-free, Compton, metal-line, molecular, fine-structure, dust collisional, cosmic ray, photoelectric and photoionization processes and selfshielding, accounting for both a metagalactic background and local stellar sources (see details below). Gas turns into stars according to a sink-particle prescription if it is self-gravitating at the resolution scale (Hopkins, Narayanan \& Murray 2013) as well as self-shielding (Krumholz \& Gnedin 2011), thermally Jeans unstable, and denser than $n_{\text {crit }}>1000 \mathrm{~cm}^{-3}$. Star particles are then considered singleage stellar populations with IMF-averaged feedback properties calculated following standard stellar evolution models (Leitherer et al. 1999): we explicitly treat mechanical feedback from stellar mass-loss (O/B and AGB winds), SNe Ia and II, as described in Paper II, and radiative feedback as described below.

For consistency and brevity, our physics study here will focus on a small subset of galaxies from Paper I, with properties in Table 1 which span a range of mass and are typical of other simulated galaxies in the same mass range. For parameter surveys we will particularly focus on two representative galaxies: a dwarf (m10q) and Milky Way (MW) mass system (m12i), which were studied in detail in Paper II.

Note that, in Paper I and Paper II, the highest resolution simulations for $\mathbf{m 1 0 q}\left(30 \mathrm{M}_{\odot}\right), \mathbf{m 1 1 q}\left(880 \mathrm{M}_{\odot}\right), \mathbf{m 1 2} \mathbf{i}$ and $\mathbf{m 1 2 m}$ $\left(7000 \mathrm{M}_{\odot}\right)$, run using our 'Default (LEBRON)' model, were a factor $\sim 8$ better mass resolution than the versions studied here. This owes to computational cost: especially with the M1 RHD solver, which requires a Courant factor limited by the (reduced) speed-of-light, it was not feasible to simulate a large parameter survey of the sort here at these extremely high-resolution levels. As shown in Paper I and Paper II, most properties here are insensitive to resolution over this range; the exception is the central 'spike' in the rotation curve of $\mathbf{m} \mathbf{1 2} \mathbf{i}$, which appears ubiquitously here, but is substantially reduced in our 'Default' method at higher resolution. We discuss explicit resolution tests below.

\subsection{Radiation hydrodynamics}

\subsubsection{Sources and frequencies}

Each star particle is a unique source, and is treated as a single stellar population with a known age $\left(t_{*}\right)$ and metallicity $(Z)$. We directly tabulate the IMF-averaged luminosity $L_{v}\left(t_{*}, Z\right)$ as a function of frequency $v$, age $t_{*}$, and metallicity $Z$ from the same stellar evolution models (STARBURST99 with a Kroupa 2002 IMF) used for SNe

\footnotetext{
${ }^{2} \mathrm{~A}$ public version of GIZMO is available at http://www.tapir.caltech.edu/ $\mathrm{ph}$ opkins/Site/GIZMO.html
}

and stellar mass-loss. Appendix A of Paper I gives approximate expressions for $L_{v}\left(t_{*}, Z\right)$.

For the physics of interest, to good approximation it is not necessary to follow a finely resolved spectrum $L_{v}$, so in our 'default' simulations we integrate into five broad bands:

(i) Hydrogen ionizing ( $L_{\text {ion }}, \lambda<912 \AA$ ), used in computing photoionization (dominated by young, massive stars).

(ii) Far-UV ( $L_{\mathrm{FUV}}, 912 \AA<\lambda<1550 \AA$ ), used for photoelectric heating (also dominated by young stellar populations).

(iii) Near-UV ( $\left.L_{\mathrm{UV}}, 1550<\lambda<3600 \AA\right)$, primarily relevant as continuum single-scattering photons (dominated by young stellar populations).

(iv) Optical/near-IR $\left(L_{\mathrm{Opt}}, 3600 \AA<\lambda<3 \mu\right.$ ), primarily relevant as continuum single-scattering photons (dominated by older stellar populations)

(v) Mid/far-IR $\left(L_{\mathrm{IR}}, \lambda>3 \mu\right)$, representing radiation absorbed and re-radiated by dust.

The spectrum $L_{v}$ of each star particle is integrated over these wavelengths to give the broad-band ( $L_{\mathrm{ion}}, L_{\mathrm{FUV}}, L_{\mathrm{UV}}, L_{\mathrm{Opt}}, L_{\mathrm{IR}}$ ). In Hopkins et al. (2012b), we compare full radiative transfer calculations in galaxy simulations using a detailed full spectrum (with $\sim 10^{7}$ frequency bins) to our simple broad-band approach, and found that discretizing the spectrum into these bands introduces $\lesssim 10$ per centlevel changes in the energy and/or momentum coupled and radiative transfer solutions.

Appendix A gives details. There we also describe a more extensive 10-band frequency network which we use for some additional tests here, which includes: hard X-ray $\left(L_{\mathrm{HX}}\right)$, soft Xray $\left(L_{\mathrm{SX}}\right), \mathrm{He}-\mathrm{II}$ ionizing $\left(L_{\mathrm{HeII}}\right), \mathrm{He}-\mathrm{I}$ ionizing $\left(L_{\mathrm{HeI}}\right), \mathrm{H}$-ionizing $\left(L_{\mathrm{HI}}\right)$, Lyman-Werner $\left(L_{\mathrm{LW}}\right)$, photoelectric $\left(L_{\mathrm{PE}}\right)$, near-UV $\left(L_{\mathrm{NUV}}\right)$, optical/near-IR $\left(L_{\text {opt }}\right)$, and multitemperature mid/far-IR $\left(L_{\mathrm{FIR}}\right.$, which tracks an effective blackbody of dynamically evolving radiation temperature).

Opacities within in each narrow band (e.g. separate $\kappa_{\text {ion }}, \kappa_{\mathrm{FUV}}$, $\left.\kappa_{\mathrm{UV}}, \kappa_{\mathrm{Opt}}, \kappa_{\mathrm{IR}}\right)$ are calculated as flux-weighted means based on the STARBURST99 mean spectra, as a function of the gas neutral fractions in the relevant states, and metallicity (assuming a constant dust-to-metals ratio); see Appendix A.

\subsubsection{Photon transport}

GIZMO includes several different RHD solvers: the direct intensity solver from Jiang, Stone \& Davis (2014), the ray-based 'LEBRON' (Locally Extincted Background Radiation in Optically Thin Networks; Hopkins et al. 2012b), and moments-based fluxlimited diffusion (FLD), first-moment (M1; Levermore 1984) and optically thin variable Eddington-tensor (OTVET; Gnedin \& Abel 2001) methods. Because exact or Monte Carlo solutions of the general 8D RT equation are simply not tractable in 'real-time' in our simulations, we study two approximate methods (both of which are computationally tractable) in this paper: (1) LEBRON, and (2) M1.

(i) LEBRON is an approximate ray-tracing method, which assumes (1) negligible light traveltimes (as most ray methods and our gravity solver also assume), and (2) local extinction in the vicinity of sources and absorbers dominates over absorption 'in between,' so the intervening transport can be approximated as optically thin. This means it trivially reduces to the exact ray-tracing RT solution in the optically thin limit, even for arbitrary numbers of sources. It also does not require a 'reduced speed of light' (RSOL) and 
Table 1. FIRE-2 simulations run to $z=0$ used for our detailed study of stellar radiative feedback here.

\begin{tabular}{llccccccl}
\hline $\begin{array}{l}\text { Simulation } \\
\text { Name }\end{array}$ & $\begin{array}{c}M_{\mathrm{halo}}^{\mathrm{vir}} \\
\left(\mathrm{M}_{\odot}\right)\end{array}$ & $\begin{array}{c}R_{\mathrm{vir}} \\
(\mathrm{kpc})\end{array}$ & $\begin{array}{c}M_{*} \\
\left(\mathrm{M}_{\odot}\right)\end{array}$ & $\begin{array}{c}R_{1 / 2} \\
(\mathrm{kpc})\end{array}$ & $\begin{array}{c}m_{i}, 1000 \\
\left(1000 \mathrm{M}_{\odot}\right)\end{array}$ & $\begin{array}{c}\epsilon_{\mathrm{gas}}^{\mathrm{MIN}} \\
(\mathrm{pc})\end{array}$ & $\begin{array}{c}r_{\mathrm{DM}}^{\text {conv }} \\
(\mathrm{pc})\end{array}$ & Notes \\
\hline $\mathbf{m 0 9}$ & $2.4 \mathrm{e} 9$ & 35.6 & $9.4 \mathrm{e} 3$ & 0.29 & 0.25 & 1.1 & 65 & Early-forming, ultrafaint field dwarf \\
$\mathbf{m 1 0 q}$ & $8.0 \mathrm{e} 9$ & 52.4 & $1.8 \mathrm{e} 6$ & 0.63 & 0.25 & 0.52 & 73 & Early-forming, dwarf spheroidal, small core \\
$\mathbf{m 1 1 b}$ & $4.3 \mathrm{e} 10$ & 92.2 & $1.1 \mathrm{e} 8$ & 2.4 & 2.1 & 2.9 & 250 & Intermediate-forming, discy, gas-rich dwarf \\
$\mathbf{m 1 1 q}$ & $1.4 \mathrm{e} 11$ & 136 & $9.8 \mathrm{e} 8$ & 4.1 & 7.0 & 1.5 & 300 & Early-forming, LMC-mass dIrr with large core \\
$\mathbf{m 1 2 i}$ & $1.2 \mathrm{e} 12$ & 278 & $1.0 \mathrm{e} 11$ & 2.3 & 56 & 1.4 & 290 & Milky-Way mass, compact disc (at low resolution) \\
$\mathbf{m 1 2 m}$ & $1.5 \mathrm{e} 12$ & 302 & $1.4 \mathrm{e} 11$ & 5.0 & 56 & 1.4 & 360 & Milky-Way mass, extended disc (at all resolutions) \\
\hline
\end{tabular}

Note. Parameters describing the FIRE-2 simulations from Hopkins et al. (2018b) that we use for our case studies. Halo and stellar properties listed refer only to the original 'target' halo around which the high-resolution region is centred. All properties listed refer to our highest resolution simulation using the standard, default FIRE-2 physics and numerical methods. All units are physical. (1) Simulation Name: Designation used throughout this paper. (2) $M_{\mathrm{halo}}^{\mathrm{vir}}$ : Virial mass (following Bryan \& Norman 1998) of the 'target' halo at $z=0$. (3) $R_{\mathrm{vir}}$ : Virial radius at $z=0$. (4) $M_{*}$ : Stellar mass of the central galaxy at $z=0$. (5) $R_{1 / 2}$ : Half-mass radius of the stars in the central $M_{*}$ at $z=0$. (6) $m_{i, 1000}$ : Mass resolution: the baryonic (gas or star) particle/element mass, in units of $1000 \mathrm{M}_{\odot}$. The DM particle mass is always larger by the universal ratio, a factor $\approx 5$. (7) $\epsilon_{\mathrm{gas}}^{\mathrm{MIN}}$ : Minimum gravitational force softening reached by the gas in the simulation (gas softenings are adaptive so always exactly match the hydrodynamic resolution or interparticle spacing); the Plummer-equivalent softening is $\approx 0.7 \epsilon_{\mathrm{gas}} .(8) r_{\mathrm{DM}}^{\text {conv }}$ : Radius of convergence in the dark matter (DM) properties, in DM-only simulations. This is based on the Power et al. (2003) criterion using the best estimate from Hopkins et al. (2018b) as to where the DM density profile is converged to within $<10$ per cent. The DM force softening is much less important and has no appreciable effects on any results shown here, so is simply fixed to $40 \mathrm{pc}$ for all runs here. The initial conditions are all publicly available at http://www.tapir.caltech.edu/ phopkins/publicICs.

properly treats photons as collisionless (so rays can intersect/cross one another). However it fails to capture shadowing, anisotropic photon diffusion (e.g. diffusion along the 'path of least resistance' in optically thick media), and the optically thin assumption means the long-range flux $\mathbf{F}_{v}$ is not strictly photon conserving (if there is, for example, a shadowing clump along a line-of-sight). ${ }^{3}$ Our LEBRON implementation is described in detail in Hopkins et al. (2018b) (see Appendix E therein).

(ii) M1 is a moments-based method which reduces to exact solutions in the infinitely optically thick regime, can capture certain shadowing effects and anisotropic photon propagation, and is manifestly photon-conserving. However it imposes strict time-step requirements which necessitate an RSOL approximation. More important, like any moments-based approximation, the closure imposed on the Eddington tensor prevents photons from behaving collisionlessly (e.g. intersecting rays 'shock' and merge, and 'new' rays isotropically diffuse out from their new location, like in FLD), so it cannot converge to correct solutions (at any resolution) in the optically thin limit for $>1$ source. This can be especially problematic in systems with many sources, like galaxies. We adopt the 'faceintegrated' formulation of M1 (Hopkins \& Grudic 2018), with the gradient treatment ${ }^{4}$ described in Rosdahl et al. (2015) - this is critical for correctly capturing the RP forces, as described below. Additional details of our M1 implementation (including e.g. how photons are isotropically 'injected' on to the grid each time-step) are given in Hopkins \& Grudic (2018), Appendix A.

Clearly, both methods have (serious) limitations. However they form a particularly useful 'pair' because their advantages/disadvantages, and regimes where they correctly converge to

\footnotetext{
${ }^{3}$ We will show below that although the LEBRON scheme is not exactly photon-conserving, the net sense of its errors are to slightly underestimate the total photon number/momentum/energy coupled to gas (at the 10 per cent level). For more explicit tests see Hopkins et al. (2012b).

${ }^{4}$ Specifically, as shown in Rosdahl et al. (2015), one obtains more accurate results with M1 in the limit where UV/ionizing photon mean-free-paths are un-resolved in neutral gas (always the case here) if we replace the explicit flux $\mathbf{F}_{v}$ with $e_{v} c \hat{\mathbf{F}}_{v}$ (the 'incident free-streaming flux') in the calculation of the RHD momentum transfer ('radiation pressure') term.
}

exact solutions, are almost exactly opposite/complementary. Thus where they give similar results, those results are likely to be robust, and where they differ, they will tend to bracket the allowed range of solutions.

\subsubsection{Radiative acceleration (radiation pressure)}

The (non-relativistic) radiative acceleration in a differential volume $d^{3} \mathbf{x}$ is just $\kappa \mathbf{F} / c$; we couple this to the gas using the 'face-integrated' formulation from Hopkins \& Grudic (2018) where this is integrated over a cell domain 'towards' each effective face:

$$
\begin{aligned}
\left.\frac{\partial \mathbf{v}}{\partial t}\right|_{v} & =\frac{\kappa_{v} \mathbf{F}_{v}}{c} \\
\left(\dot{\mathbf{p}}_{v}\right)_{a b} & \equiv \int_{\Delta \mathrm{Vol}_{a}} d^{3} \mathbf{x} \frac{\rho \kappa \mathbf{F}_{v}(\mathbf{x})}{c} \Theta\left(\mathbf{x}, \hat{\mathbf{F}}_{v}, c \mathbf{A}_{a b}\right),
\end{aligned}
$$

where $\Theta=1$ if the flux vector $\hat{\mathbf{F}}_{v}$ at point $\mathbf{x}$ (within the domain of cell $a$ ) points 'towards' face $\mathbf{A}_{a b}$ (of the faces surrounding $a$, it is the first one intercepted by the ray $\hat{\mathbf{F}}_{v}$ ), and $\Theta=0$ otherwise.

As shown in Hopkins \& Grudic (2018), older 'cell-integrated' or 'cell-centred' methods - where equation (1) is simply integrated over the whole cell volume (or evaluated at the cell centre) instead of at cell faces - artificially suppress the radiation pressure force by at least an order of magnitude if the mean-free-path of photons around sources is un-resolved. For ionizing photons, resolving the mean free path in neutral gas at the location of a star particle would require an un-achievable mass resolution $m_{i} \lesssim 10^{-10} \mathrm{M}_{\odot}\left(n / 100 \mathrm{~cm}^{-3}\right)^{-2}$.

\subsubsection{Radiative heating and indirect feedback}

Radiative heating/cooling and photoionization rates follow standard expressions (all given explicitly in Paper I; App. B). These include (among other processes): photoionization (HI, HeI, HeII), photoelectric heating, dust collisional heating/cooling, Compton heating/cooling. Each of these depends on the radiation energy density $e_{v}$ in some band[s]: these are taken from the RT solution and used directly in the appropriate heating/cooling functions. If our 'default' runs do not include RT in some band (e.g. X-rays, only followed in our extended set, or if we 'turn-off' radiative transfer in 
a given band), then we assume a universal Milky Way background for the term in the heating/cooling routine.

\subsubsection{The metagalactic UV background}

For the sake of consistency with our previous FIRE simulations and considerable historical work, we do not explicitly solve for the UV background from a set of 'sources' - this means we are not self-consistently solving the RT equations for the photons in the metagalactic UV background. Doing so is highly non-trivial in our zoom-in simulations, since there are only a small number of galaxies inside the high-resolution region (vastly smaller than the $\gg 100 \mathrm{Mpc}$ scales needed to correctly capture the collective generation of the background), and the 'boundaries' of the hydrodynamic grid on which the M1 equations are solved for the RHD are constantly changing and irregular (so even a "photon inflow boundary condition' is not well-defined). Instead, we follow most previous galaxy-formation simulations and assume a spatially uniform but redshift-dependent UV background tabulated from Faucher-Giguère et al. (2009), with self-shielding accounted for via local attenuation with a Sobolev approximation (see Hopkins et al. $2018 \mathrm{~b}$ for details). After this self-shielding correction, the remaining UVB spectrum is added to the explicitly followed relevant ionizing RT band intensities, for use in computing e.g. photoheating and ionization states above.

\section{RESULTS}

\subsection{Overview: Net effects of radiative feedback on galaxy properties}

Figs 1 and 2 compares the effects of radiative FB as a function of galaxy mass. For each galaxy IC in our sample (ranging from ultrafaint to MW mass), we compare four simulations: (1) 'Default (LEBRON)' - this uses the 'standard' FIRE-2 radiative FB implementation from Paper I, including five spectral bands accounting for photoionization (by both local sources and the UVB), photoelectric heating, continuum absorption (and associated radiation pressure/momentum transfer) by dust in near-UV, optical/NIR, and mid/far-IR, and re-emission in the IR. (2) 'Default (M1)' - this uses the same source functions, rates, etc., but replaces the photon transport step with the M1 algorithm. (3) 'No Local RHD' - this disables all local sources, i.e. star particles emit no radiation, but the UVB remains. (4) 'No Radiative FB' - this disables local sources and the UVB.

We run each simulation to $z=0$, then show the resulting star formation history (for all stars which reside within the $z=0$ galaxy), stellar mass growth history, ${ }^{5}$ mean stellar metallicity as a function of time, and $z=0$ (spherically averaged) mass density profile of baryons and dark matter (and stellar effective radius), and the $z=0$ circular velocity curve. The resolution of each simulation is labelled. Additional details about how each quantity are computed are in Paper I.

Removing radiative FB entirely, we see order-of-magnitude larger SFRs and stellar masses in dwarfs. ${ }^{6}$ Not surprisingly, most of

\footnotetext{
${ }^{5}$ We plot the archaeological mass growth, i.e. the stellar mass formed at each time, which at $z=0$ resides within the virialized halo.

${ }^{6}$ Note that, for the lowest mass dwarfs $\mathbf{m 0 9}$ and $\mathbf{~ m 1 0 q}$, the higher stellar and baryonic mass associated with removing all radiative FB actually correlates with lower central circular velocity. This effect owes to the formation of a
}

this effect comes from the 'external' UVB. With this fixed, ultrafaint dwarfs (e.g. m09) are quenched or 'starved' of new gas by the UVB; as a result (also given their very low stellar masses) the 'local' radiative $\mathrm{FB}$ generated by stars is subdominant. In more massive dwarfs, the 'local' radiative FB has an important effect 'smoothing out' feedback. Because it provides a 'gentle' feedback mechanism (e.g. keeping gas warm, which can maintain $Q>1$ and prevent runaway gravitational instability in a dwarf galaxy; see Shetty \& Ostriker 2008; Kannan et al. 2014b; Rosdahl et al. 2015) and helps disrupt GMCs before they turn most of their mass into stars (Murray et al. 2010; Harper-Clark \& Murray 2011; Lopez et al. 2011; Hopkins et al. 2012b; Colín et al. 2013; Grudić et al. 2018a), it makes star formation less 'violent.' Without local radiative FB, m10q, for example, undergoes complete 'self-quenching' (gas collapses and 'overshoots,' forming too many $\mathrm{SNe}$, which then blow out all the gas, and no stars form in the last $\sim 10 \mathrm{Gyr}$, the galaxy has no gas, and has a highly suppressed metallicity). At still higher masses the potential becomes deeper, so these effects are progressively less prominent. ${ }^{7}$

In detail: we show explicitly below that star formation is more strongly clustered without 'early' feedback to disrupt clouds, but this is already well-established from much higher resolution simulations of individual GMCs, which show they collapse more efficiently and turn much more of their mass into stars in a single free-fall time, without early radiative feedback from $\mathrm{H}$ II regions and radiation pressure (see e.g. Harper-Clark \& Murray 2011; Colín et al. 2013; Howard et al. 2016; Kim et al. 2017; Grudić et al. 2018b). ${ }^{8}$ We see this below directly in the galaxy morphologies (Fig. 7, Section 4.2), amplitude of the bursts (Fig. 1), and in our previous studies showing the efficiency of star formation in individual GMCs (Hopkins et al. 2012b; Corbett Moran, Grudić \& Hopkins 2018; Kim et al. 2018). In turn, many idealized studies of SNe-driven outflows have shown that enhancing the clustering (in time or space) of star formation (hence young stars and $\mathrm{SNe}$ explosions) leads to stronger outflows as the clustered explosions more easily produce superbubbles and chimneys, and lose less energy to radiation (see e.g. Walch et al. 2015; Gentry et al. 2017; Fielding, Quataert \& Martizzi 2018, and references therein). We see this reflected indirectly in the suppressed stellar masses and total baryonic masses (by definition inversely proportional to the outflow 'mass loading factor' averaged over cosmic time) within $\sim 1-10 \mathrm{kpc}$ in our dwarfs without early or local radiative FB (see e.g. $\mathbf{~ m 1 0 q}$ and $\mathbf{m 1 1 b}$ in Figs 1 and 8). Most dramatic are the 'self-quenching' events discussed above. A more detailed study of

'cored' dark matter profile via stellar feedback, the efficiency of which is a strong function of the stellar-to-dark matter mass ratio (see detailed studies in Oñorbe et al. 2015; Chan et al. 2015, 2018).

${ }^{7}$ The detailed SFRs versus time of the dwarfs, in particular, are quite stochastic and subject to large run-to-run fluctuations in e.g. burst timing: as shown in Paper I, systematic differences in mass or trends versus time are much more robust. For example, $\mathbf{m 1 0 q}$ at some resolutions in Fig. 3 briefly ceases forming stars for $\sim 1$ Gyr before 'rejuvenating' (this occurs at high resolution near $z \sim 0$, with rejuvenation at $z \sim 0.04$ ). But other runs of m10q with seeded minor perturbations in Su et al. (2018) show the timing and depth of this specific burst-quench cycle are stochastic, while e.g. the total stellar mass is robust to $\lesssim 0.1 \mathrm{dex}$.

${ }^{8}$ Worth noting here, simulations using the same physics as those here but simulating single clouds with resolution reaching $\sim 0.01 M_{\odot}$ actually find cloud-lifetime integrated star formation efficiencies and radiative feedback efficiencies in good agreement with our galaxy-scale simulations (compare Hopkins et al. 2012b; Oklopčić et al. 2017; Grudić et al. 2018b, 2019). 

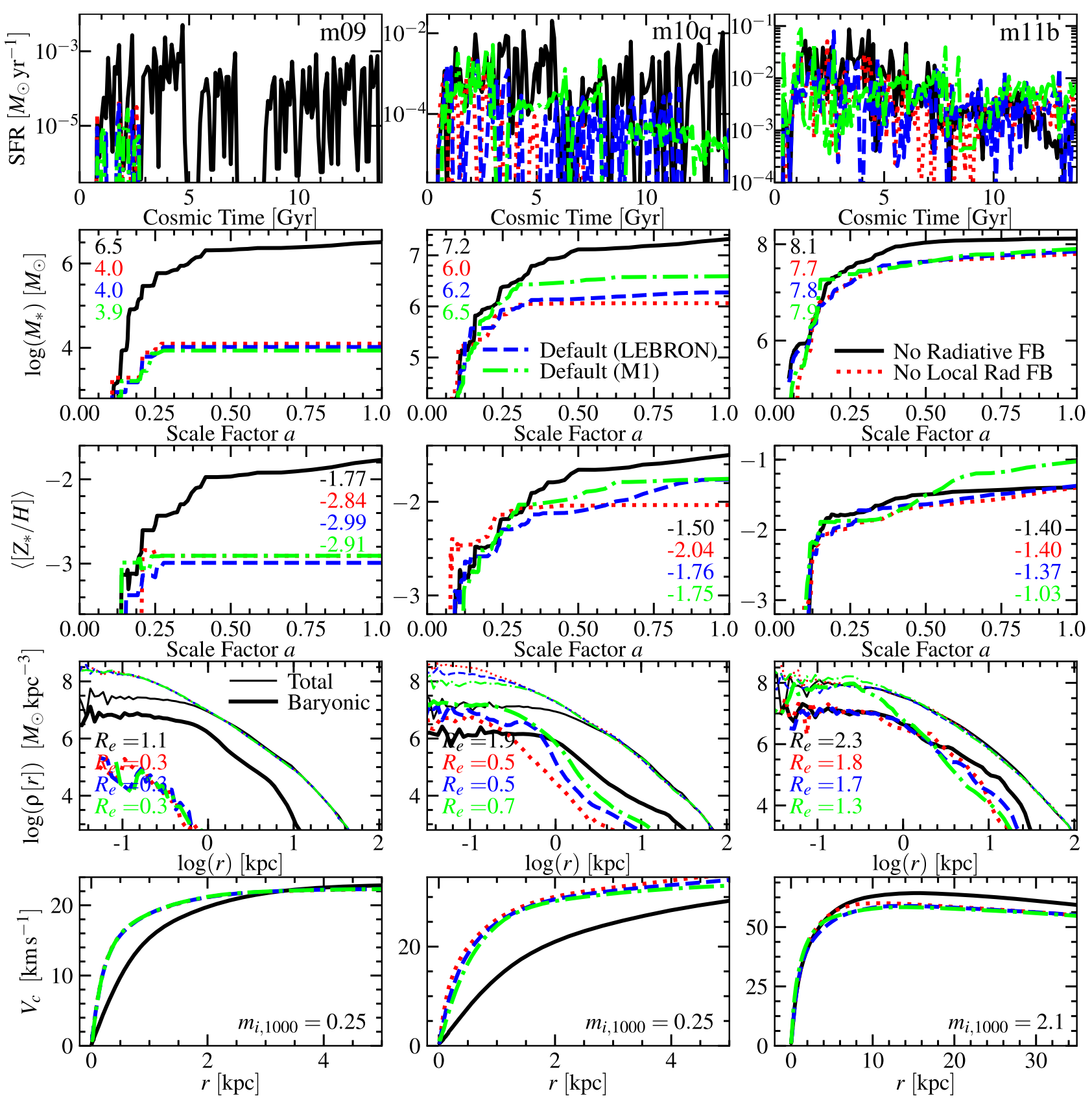

Figure 1. Effects of radiative feedback on gross galaxy properties in cosmological simulations (dwarfs; continued in Fig. 2). Top: Star formation history (averaged in $100 \mathrm{Myr}$ intervals) of the primary $(z=0)$ galaxy from Table 1. Second: Total stellar mass in box (dominated by primary) versus scale factor $(a=$ $1 /(1+z))$. The value at $z=0$ for each run is shown as the number in the panel. Middle: Stellar mass-weighted average metallicity versus scale factor $(z=0$ value shown). Third: Baryonic (thick) and total (thin) mass density profiles (averaged in spherical shells) as a function of radius around the primary galaxy at $z=0$. Number is the stellar effective ( $1 / 2$ mass) radius at $z=0$. Bottom: Rotation curves (circular velocity $V_{c}$ versus radius) in the primary galaxy. Value $m_{i, 1} 1000$ of the mass resolution is shown. In each, we compare variations from Section 4.1: (1) Default (LEBRON): The standard FIRE-2 radiative FB implementation including photoionization, photoelectric heating, near UV/optical/IR single-scattering and re-emission with multiple-scattering in the IR, using the LEBRON radiative transport algorithm. (2) Default (M1): This uses the same default source functions, opacities, etc., for all radiation quantities, but replaces the photon transport with the moments-based M1 RHD algorithm. (3) No Local Rad FB: Removes all radiative FB from stars in the simulation, but keeps the (uniform) metagalactic UVB. (4) No Radiative FB: Removes all radiative FB (including the UVB). For dwarfs, the UVB has a large effect; this remains significant even up to LMC-like masses. Removing local radiative FB leads to more 'violent' SF (m10q 'overshoots' and 'self-quenches' at $z \sim 2$ and has almost no gas, and no SF, at later times, without radiative FB, producing a lower total stellar mass) in dwarfs, and somewhat more dense centrally concentrated SF in massive galaxies (Fig. 2). M1 and LEBRON algorithms produce qualitatively similar effects, though quantitatively effects appear somewhat stronger in the M1 runs. 

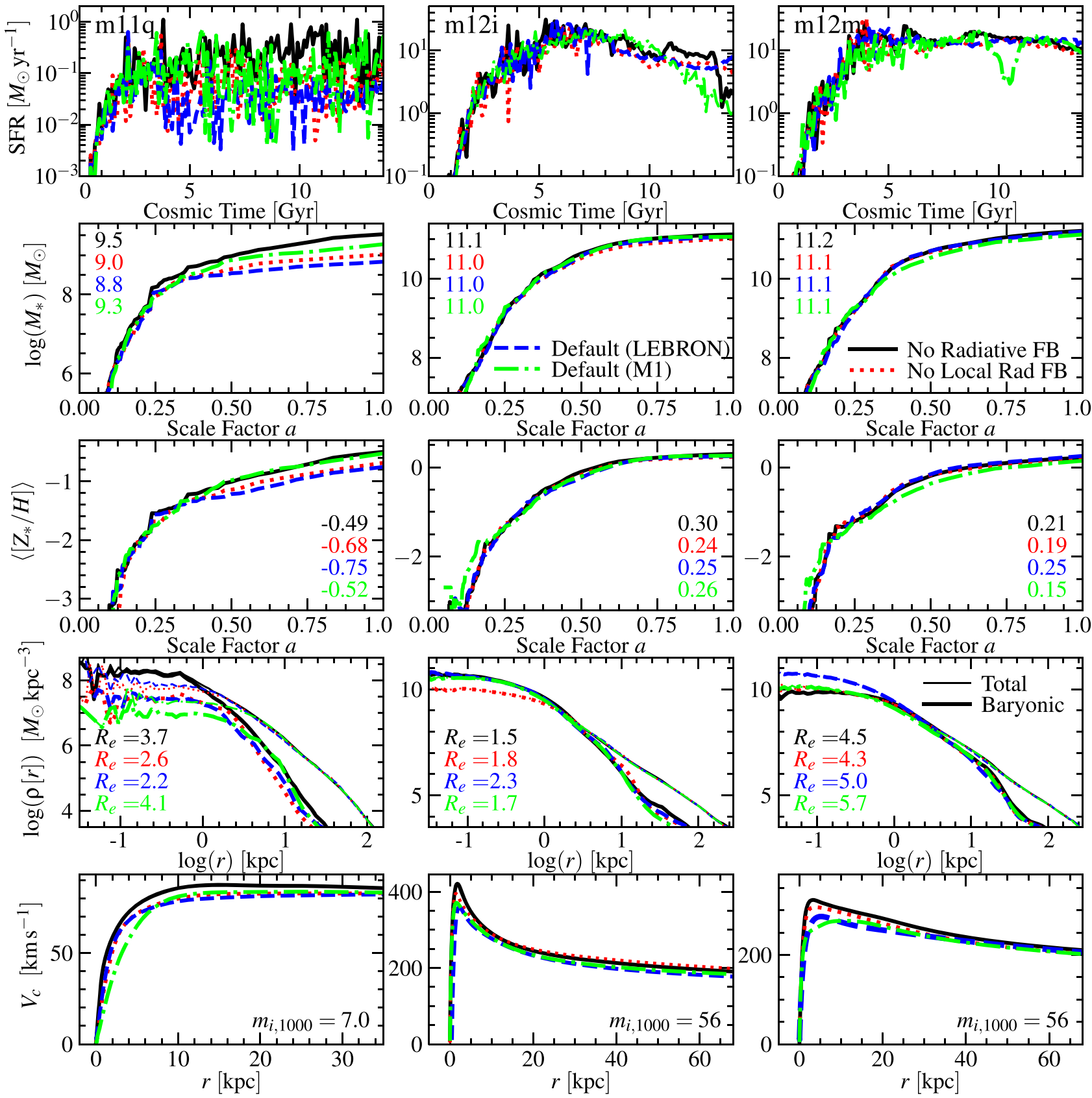

Figure 2. Fig. 1, continued for more massive galaxies. Removing local radiative FB leads to somewhat more dense centrally concentrated SF in massive galaxies. Again, M1 and LEBRON algorithms produce qualitatively similar effects, though quantitatively effects appear slightly stronger in the M1 runs.

the wind mass-loading factors and how they vary in-and-out of 'bursts' (with different feedback physics included or excluded) is in preparation, but for some results, see Muratov et al. (2015).

By MW-mass, the UVB has only weak effects (on the primary galaxy - a more detailed study of how the UVB alters e.g. ultrafaint galaxies in the Local Group, as satellites of the MW, will be the subject of future work). And overall, the effects of local radiative FB on gross properties of the galaxy (SFRs, stellar masses, metallicities, baryonic mass profiles, in Figs 2 and 3) are generally weaker in all respects (though we show below this is not true for their detailed small-scale morphology/structure). This is expected: the galaxies have deep potential wells with escape velocities $\gg 100 \mathrm{~km} \mathrm{~s}^{-1}$, so photoionization heating does not suppress accretion or appreciably 'thicken' the disc, they have higher gas densities so cooling times are relatively short (warm gas can still radiate efficiently and collapse to form stars), and winds (driven by radiation as well as $\mathrm{SNe}$ ) simply become much less efficient (the baryonic mass of the galaxy at MW masses is an appreciable fraction of the total supply $\sim f_{\text {baryon }} M_{\text {halo }}$, see Paper I) so it is simply the case that all feedback effects are much weaker.

However, in our MW-mass runs some modest 'excessive burstiness' without local radiative FB is still evident at early times (when the galaxy is a dwarf; see e.g. SFRs for m12i at $t<6$ Gyr in Figs 2 and 3), but we also see that the runs without local radiative FB have slightly higher central rotation curve peaks - this is more obvious in Fig. 3 (and in the tests presented in Hopkins et al. 

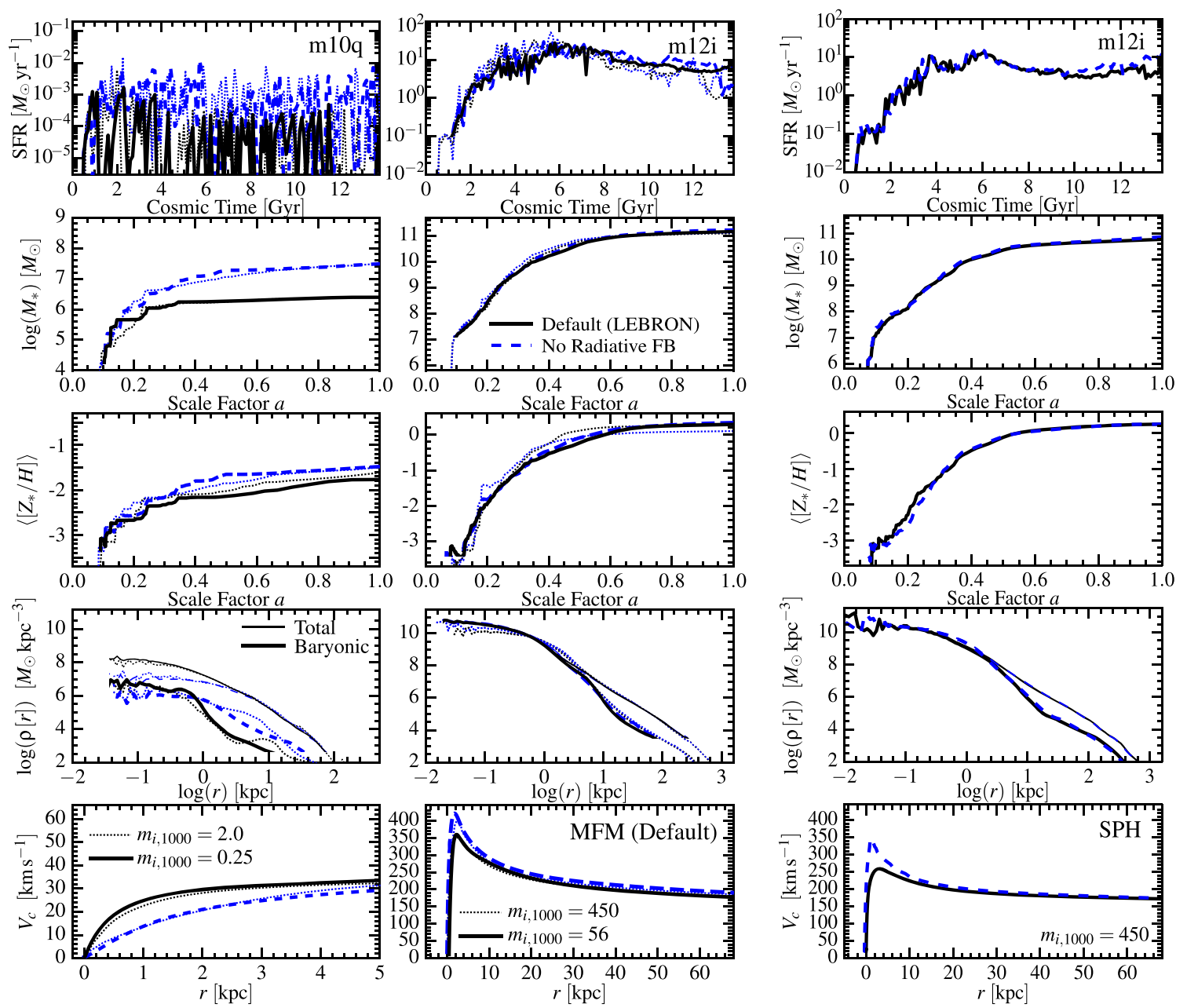

Figure 3. Effects of radiative FB on $\mathbf{m} 10 \mathbf{q} \& \mathbf{m} \mathbf{1 2} \mathbf{i}$ as Figs 1 and 2. Left-hand panel: We show $\mathbf{m} 10 \mathbf{q}$ and $\mathbf{m} 12 \mathbf{i}$ at lower and higher resolution, as in Fig. 1. Right-hand panel: We show m12i re-run at the lower resolution using the smoothed-particle hydrodynamics implementation used in the original FIRE-1 simulations (Hopkins et al. 2014), as described and compared in detail in Hopkins et al. (2018b). The qualitative effects of 'turning off' RFB are similar across resolution levels and hydro solvers. However SPH, at low resolution, produces a slightly less-compact and lower-SFR m12i (the difference decreases at higher resolution) - discussed in detail in Paper I - so the relative effect of radiation suppressing the steep central rotation curve excess is more obvious.

2014). Recall, m12i in particular exhibits a sharp central rotation curve 'spike' at this (relatively low) resolution, so the relative effect is small. As discussed in detail in Paper I, this feature in $V_{c}$ is sensitive to both resolution and hydrodynamic methods; which is why the effects of radiation on this feature are more obvious in Fig. 3. These galaxies have high central densities, so failure to destroy GMCs before SNe explode means those explosions would occur in dense environments, suppressing SNe bubble overlap and therefore expulsion of material from dense galaxy centres in galactic superwinds - we see this below in a suppressed hot gas content within the ISM (Figs 4 and 5). Note that the lack of local/early FB still produces more strongly clustered SF (see Section 4.2 below); but unlike in dwarfs (1) the densities are much higher in the galactic nuclei, so $\mathrm{SNe}$ become less efficient and bubble overlap is more challenging (as the $\mathrm{SNe}$ cooling radii scale $\sim \operatorname{pc}\left(n / 10^{4} \mathrm{~cm}^{-3}\right)^{-1 / 3}$; see Paper II), (2) optical depths to radiation pressure are much larger (given higher densities and metallicities, compared to dwarfs), and (3) the dynamical times in galaxy centres $\sim 2 \operatorname{Myr}(R / \mathrm{kpc})\left(400 \mathrm{~km} \mathrm{~s}^{-1} / V_{c}\right)$ become shorter than the time over which most SNe explode $(\sim 30 \mathrm{Myr})$, so early feedback becomes more important in regulating against runaway SF (Torrey et al. 2017; Grudić et al. 2018b). In short, in both dwarfs and MWmass systems, local radiative FB is most important in suppressing SF efficiencies on small spatial/time-scales (and/or high density scales). As we show below, for fixed gas properties, the radiation also has an important role regulating how fast the gas turns into stars in massive systems on the disc scale, but that is hidden here because faster star formation at early times exhausts the gas, leading to lower supply at late times.

Fig. 3 compares 'No Radiative FB' and 'Default (LEBRON)' models at different resolution, and using a different hydrodynamic solver (the 'pressure' formulation of smoothed-particle hydrodynamics, i.e. 'P-SPH' from Hopkins 2013a as used for the FIRE-1 simulations, instead of our default meshless-finite mass or MFM solver). As noted above, Paper I contains extensive discussion and comparison of how the hydrodynamic solver and resolution influence our results; our only purpose here is to illustrate that the qualitative effects of radiation are independent of both resolution and hydrosolver, even if the quantitative details differ. It is particularly worth noting in this context that Paper I, Guszejnov et al. (2017), and Guszejnov et al. (2019) all consider explicit resolution studies of ISM phase structure including GMC mass 


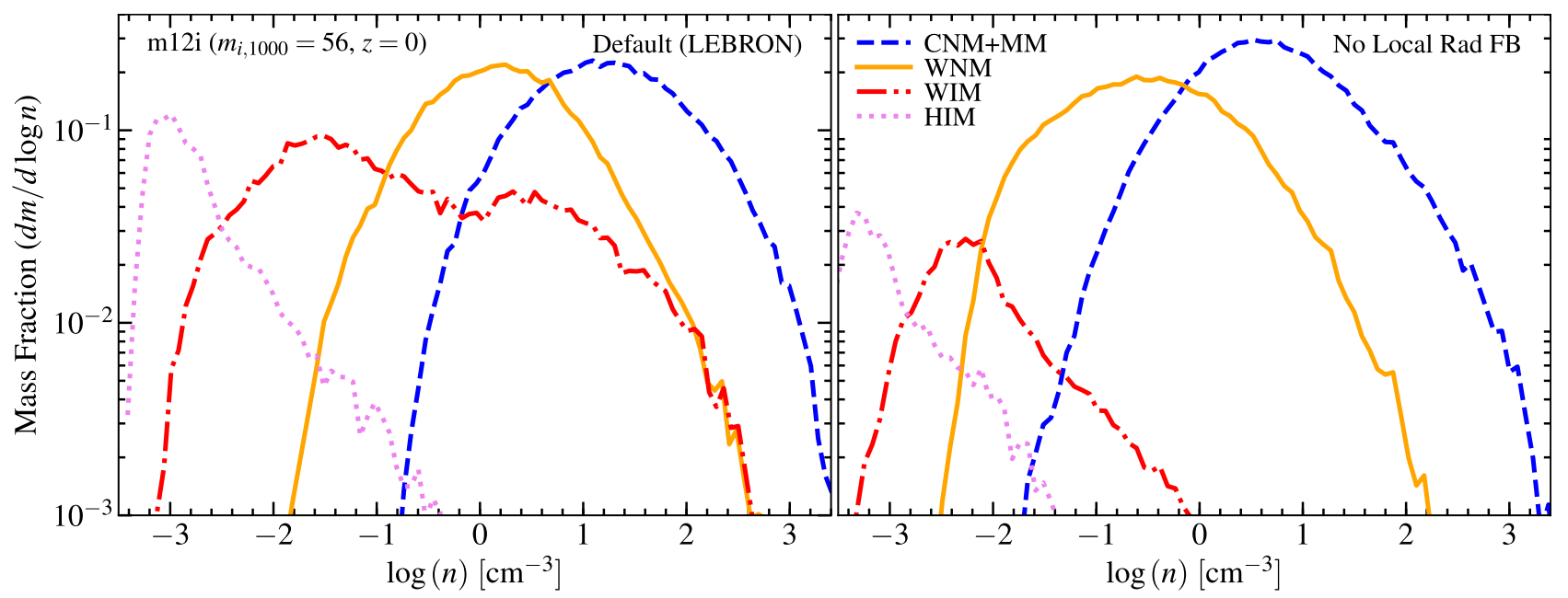

Figure 4. Effect of radiative FB on the distribution of ISM gas phases. Differential mass fraction ( $\mathrm{d} m / \mathrm{d} \log n$, normalized to the total gas mass) of gas within $<20 \mathrm{kpc}$ of the galactic centre of $\mathbf{m 1 2 i}$ at $z=0$, in our 'Default (LEBRON)' (left-hand panel) and 'No Local Rad FB' (right-hand panel) runs. We separately show the cold $(T<1000 \mathrm{~K})$ neutral and molecular medium $(\mathrm{CNM}+\mathrm{MM})$, warm $(T>1000 \mathrm{~K})$ neutral medium $(\mathrm{WNM})$, warm $\left(T<10^{6} \mathrm{~K}\right)$ ionized $(\mathrm{WIM})$ medium, and hot $\left(T>10^{6} \mathrm{~K}\right)$ ionized medium (HIM). Ionization states are taken directly from the self-consistent values in-code. In 'Default,' the distribution of gas in different phases and densities broadly agrees with canonical Milky Way values (e.g. Draine 2011). In 'No Local Rad FB,' the mass of ionized gas in the ISM decreases by a factor $\sim 10$; there are no H II regions (no WIM at high $n \gg 1 \mathrm{~cm}^{-3}$ ); lack of (local) photoionization and photoelectric heating means the overall mass fraction of CNM is larger (and it dominates the mass budget at lower densities $n \gtrsim 1 \mathrm{~cm}^{-3}$ ); the WNM persists (because cooling is inefficient below $\sim 8000 \mathrm{~K}$ ) but dominates even at extremely low densities $n \sim 10^{-2} \mathrm{~cm}^{-3}$ (approximately the density where the ISM becomes self-shielding against the metagalactic UV background). Hot gas is also suppressed owing to the less efficient preprocessing of GMCs (reducing their densities, hence increasing cooling times) before SNe explode within them.
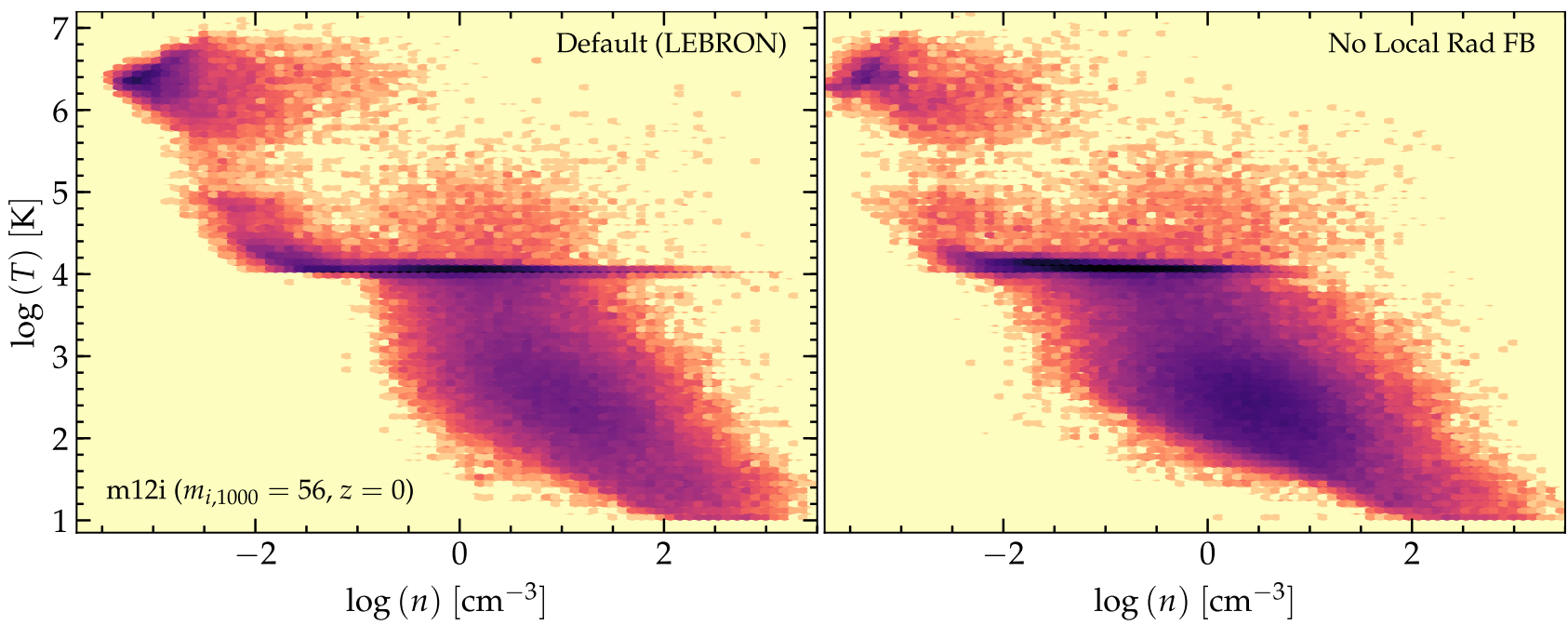

Figure 5. Effect of radiative FB on the distribution of ISM gas phases, as Fig. 4, continued. Here we show a 2D histogram of temperature and density (no ionization information) at the same time, weighted by the log of the total gas mass in each pixel (darker is larger gas mass per unit density temperature). The same trends are evident, but the decrease in the WIM at intermediate densities from Fig. 4 is less obvious, because this is mostly a change in ionization state as WIM becomes WNM without local radiative FB.

functions, the cloud linewidth-size relation, and size-mass relations (hence typical cloud surface densities and opacities), and show that these are actually quite robust over factor $>100$ improvements in resolution. Although there is certainty unresolved substructure in the cold gas at any resolution level, quantities like the GMC mass function and size-mass relation are not modified at the resolved, largest masses (which contain most of the star formation, feedback, and cold gas mass in galaxies; see Solomon et al. 1987; Blitz 1993; Murray 2011; Rice et al. 2016) - rather increasing the resolution simply extends these to smaller and smaller clouds (which do not contribute much to the total mass or star formation budget).

Figs 4-7 compare the phase distributions and visual morphologies of a subset of these runs. We specifically examine how radiative feedback alters the temperature distribution of dense, cool gas in the halo, and how the combination of radiative and other 'early' FB channels alters the visual morphology of the galaxies. Note that although the visualizations of morphology shown are mock images (i.e. light-weighted), the differences in morphology persist if we make a simple stellar mass-weighted map. Though the effect is 


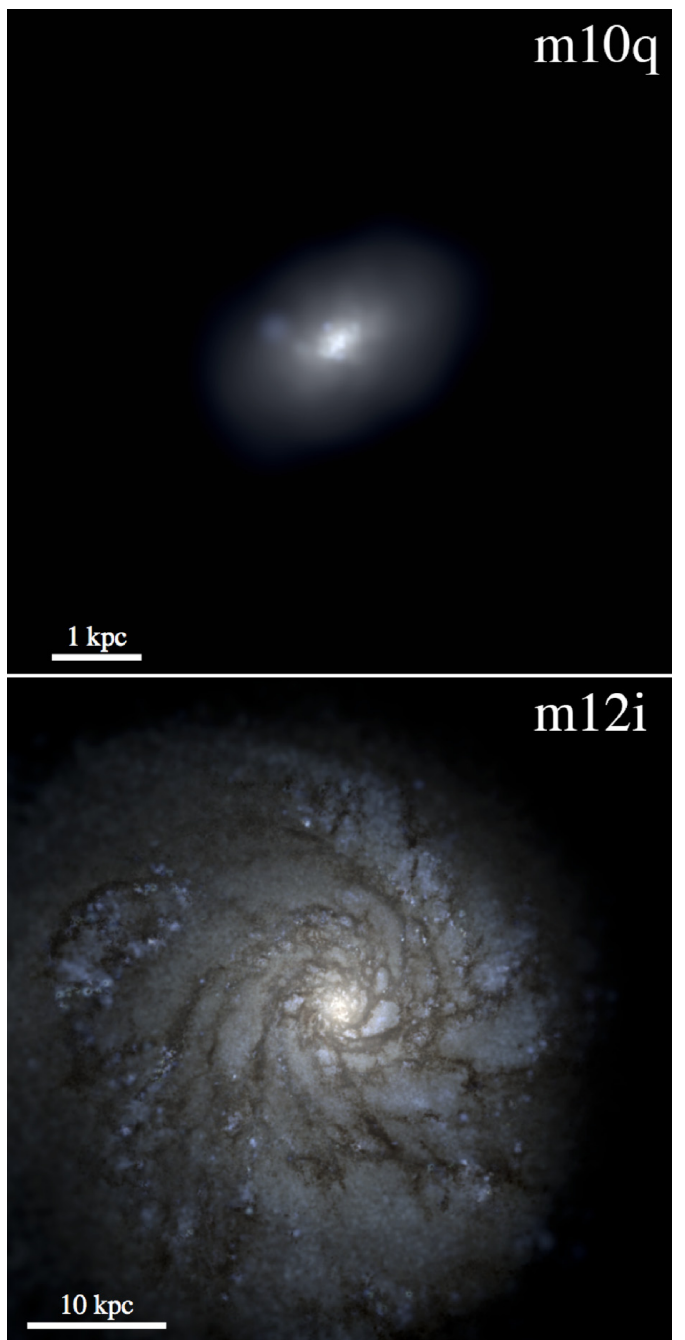

Figure 6. Mock image of our highest resolution 'Default (LEBRON)' $\mathbf{m} 10 \mathbf{q}$ run and $\mathbf{m} 12 \mathbf{i}$ run at $z=0$. These are compared in Figs 1-14. $\mathbf{m 1 0 q}$ is a dSph with no coherent morphological structure - as a result, there is no discernable difference in its visual morphology in our different runs. Moreover, we see that there is essentially no dust obscuration, owing to low gas densities and (more importantly) very low metallicities $\sim 0.01 Z_{\odot}$, so only ionizing photon absorption by neutral gas produces large effects. m12i exhibits substantial differences (see e.g. Fig. 7) owing to its thin-disc morphology; the run here shows the young stars are mostly, at late times, forming in spiral arms which are highly dust-obscured, where most of the absorption studied in Figs 13 and 14 occurs (these lanes/clouds have gas surface densities $\sim 50-100 M_{\odot} / \mathrm{pc}^{-2}$ ).

subtle, we see the MW-mass runs without local radiative FB produce somewhat less-discy morphologies. Runs without radiation have a substantially different gas temperature distribution, as expected (e.g. $\mathrm{H}$ II regions do not exist). ${ }^{9}$ The lack of 'pre-processing' of GMCs by $\mathrm{H}$ II regions and winds also means that the clouds have higher densities when SNe explode, which means they, in turn, have shorter cooling times, producing less hot gas (see e.g. the results in noncosmological simulations in Hopkins, Quataert \& Murray 2012a or in idealized experiments in Haid et al. 2016). Note that for these

\footnotetext{
${ }^{9}$ In Fig. 4, we include all ionized gas with $T<10^{6} \mathrm{~K}$ in the 'warm ionized' medium, but because it is weighted by the ionized gas mass, material with $T \ll 10^{4} \mathrm{~K}$ contributes negligibly.
}

runs we focus on MW-mass systems, where the differences are most pronounced. The low-mass dwarfs all have irregular/spheroidal morphologies - the fact that small dwarfs $\left(M_{\text {halo }} \ll 10^{11} \mathrm{M}_{\odot}\right)$ tend to be spheroidal or irregular in morphology, and that the stellar orbits are dispersion (as compared to rotation)-dominated, is not surprising (this is expected for many reasons in systems with $V_{c} \ll 100 \mathrm{~km} \mathrm{~s}^{-1}$ for many reasons; see e.g. Wheeler et al. 2017). Indeed previous studies have shown these morphological traits remain even if we remove all stellar feedback (Hopkins et al. 2014, 2018b, a), or add additional physics such as magnetic fields and cosmic rays ( $\mathrm{Su}$ et al. 2017; Hopkins et al. 2019). The 'quantitative photometric morphologies,' on the other hand, defined using e.g. colours or Sersic indices may vary, however, as we have shown in Fig. 1 that the central stellar mass profile and SFRs do vary.

\subsection{The role of 'Early' feedback}

Fig. 8 compares runs where we remove all local radiative FB and all other 'early FB' (FB from massive stars before they explode). In our implementation, this includes radiation from massive stars, as well as stellar mass-loss in $\mathrm{O} / \mathrm{B}$ winds from massive stars before they explode (see Paper I for details). These runs are also compared in Figs 4-7.

The effects described above in Section 4.1 become much more dramatic without any 'early FB.' Every galaxy forms many more stars early, at redshifts $z \gtrsim 6-7$ (while a small fraction of their $z=0$ mass, this makes them order-of-magnitude more massive at these times). The SFR 'spikes' at much higher values and these overviolent bursts produce 'self-quenching' in $\mathbf{m 1 0 q}$ and $\mathbf{m 1 1 b}$. Even m11q, an LMC-mass system, essentially self-quenches for $\sim 5-10 \mathrm{Gyr}$, although it recovers below redshift $z \lesssim 0.5$. In all cases these 'blowouts' strongly suppress the metallicity (by $\sim 0.3-$ $0.4 \mathrm{dex}$ ), pushing the galaxies significantly below the observed stellar mass-metallicity relation (compare Ma et al. 2016a). We also see in the baryonic mass profiles that the systems are significantly more baryon-poor out to $\gtrsim 10 \mathrm{kpc}-$ i.e. they have ejected most of their gas (see e.g. the suppression of $\rho_{\text {baryon }}$ from $\sim 1-10 \mathrm{kpc}$ with 'No Early FB' compared to 'Default' in the second-from-bottom panels in Fig. 8). These effects are also evident in Figs 7 and 6.

In $\mathbf{m} 12 \mathbf{i}$, these effects are proportionally smaller, but in fact we have to stop the run at $z \approx 1$ as the time-steps become extremely small ( $\sim 1$ yr). The reason is obvious in Fig. 7: without any early FB, dense GMCs collapse on a time-scale faster than their stellar evolution time-scale (at densities $n \gtrsim 1000 \mathrm{~cm}^{-3}$ typical of dense GMCs, the free-fall time is $\lesssim 1 \mathrm{Myr}$ ). As shown in many previous, much higher resolution studies of individual GMCs (see references in Section 1 or Grudić et al. 2018b) or 'zoom-ins' of GMCs in galaxy simulations (Kim et al. 2018), this leads to the GMC turning most of its mass into stars, and leaves behind a very dense, bound remnant. As a result, most of the stellar mass is composed of extremely dense bound star clusters (for comparison, $\lesssim 1$ per cent of the stellar mass in the MW is in such objects; see e.g. Harris 1996; Peng et al. 2008). Because the galaxy is essentially assembling hierarchically from 'minor mergers' of dense collisionless stellar clumps, its morphology has no recognizable disc and little angular momentum.

This is consistent with previous studies, which have shown that without some form of 'early FB,' galaxy-scale simulations disagree at the order-of-magnitude level with observations of quantities such GMC mass functions, size-mass, and virial parameter scalings, and GMC lifetimes (Hopkins et al. 2012b; Oklopčić et al. 2017; Grudić et al. 2018b), as well as the ratio of various dense gas tracers in the 

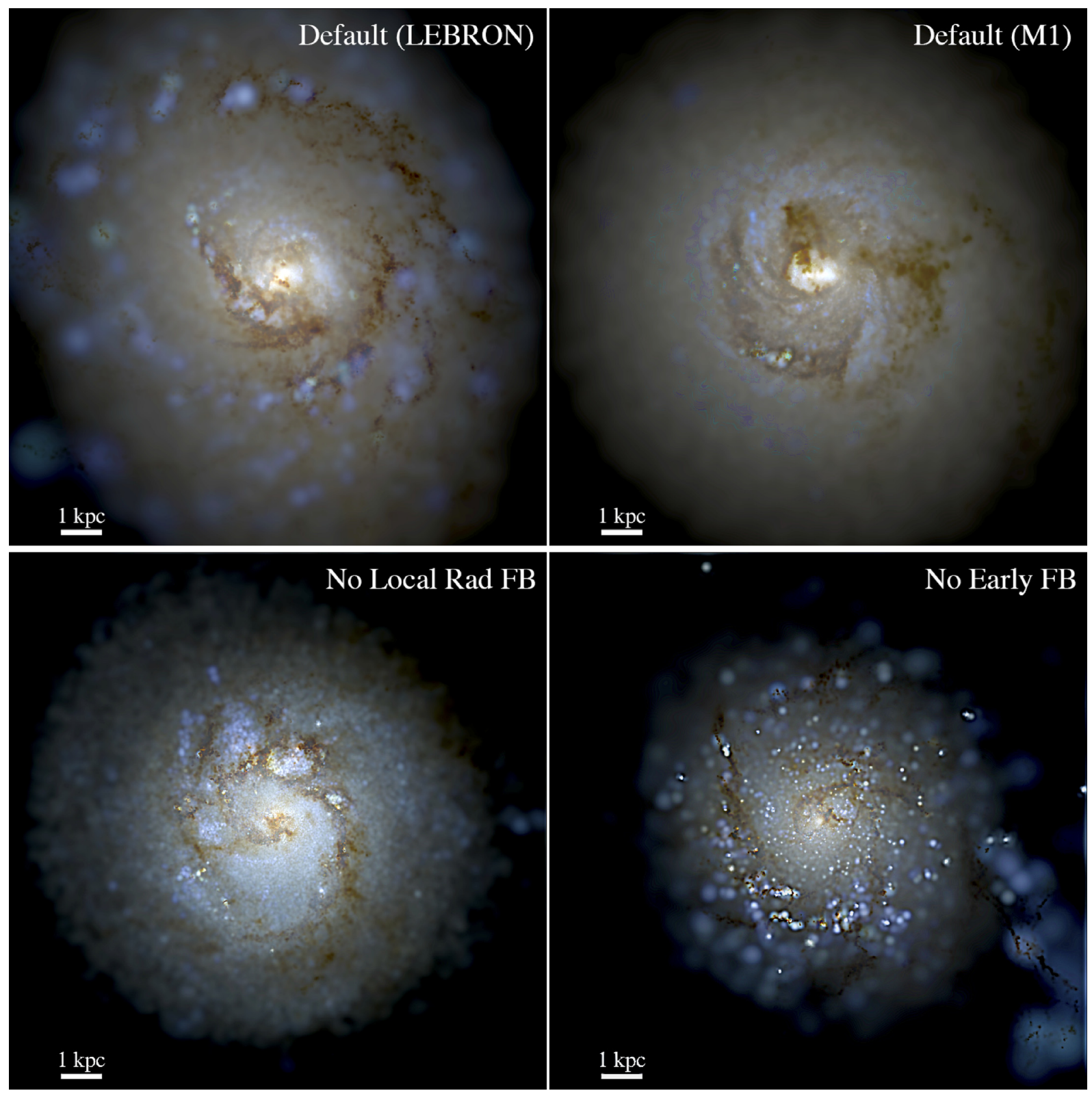

Figure 7. Mock images of our Milky Way-mass $\mathbf{m 1 2 i}$ galaxy $\left(m_{i, 1000}=56\right)$ at $z \approx 0.9$ (the lowest redshift to which all runs were run). We compare the 'Default (LEBRON)' and 'Default (M1)' (top) and 'No Local Rad FB' and 'No Early FB' runs (bottom) from Fig. 8. While the details of e.g. disc thickness and spiral arm structure differ between LEBRON and M1, these are highly time-variable and subject to stochastic run-to-run variations. Without local radiative FB, the galaxy size and mass and integrated colour are similar, but the spiral structure is significantly less obvious owing to the more-bursty episodes blowing out gas, and there are more small, discrete star clusters. Without any early FB, the galaxy morphology and stellar mass is entirely dominated by hypercompact star clusters. Although some vague spiral-like structure appears here it is an artefact of a recent merger - the star clusters which dominate the stellar mass are mostly on nearly-radial orbits.

ISM (e.g. CO versus HCN; see Hopkins et al. 2013). Similarly, other studies have shown that 'early FB' has an order-of-magnitude effect on ionizing photon escape fractions (as, absent any such FB to create channels in GMCs before the most massive stars - which produce almost all the ionizing photons - explode, the escape fraction is negligibly small; see Ma et al. 2015, 2016b).

We emphasize that, in Paper I and Paper II, as well as several of the references above, it was shown that disabling only O/B massloss (or all stellar mass-loss), while retaining radiative FB from both photoionization and radiation pressure, produces only minor effects (significantly smaller, in fact, than removing radiative FB while retaining stellar mass-loss). It is therefore not the case that the O/B winds 'dominate. ${ }^{10}$ Rather, it seems that the different 'early FB' channels: photoionization heating, single-scattering radiation

\footnotetext{
${ }^{10}$ Moreover, as briefly noted in Grudić et al. (2018b), the default FIRE scaling (used here) from an older version of STARBURST99 for O/B massloss rates extrapolates, at low metallicities, to higher mass-loss rates than given by other more recent stellar evolution models (particularly those favoured by the massive black hole mergers in LIGO; see discussion in Lamberts et al. 2016, 2018). We have experimented (not shown here) with a more recent model, which has weak effects overall (see Paper I), but this
} 

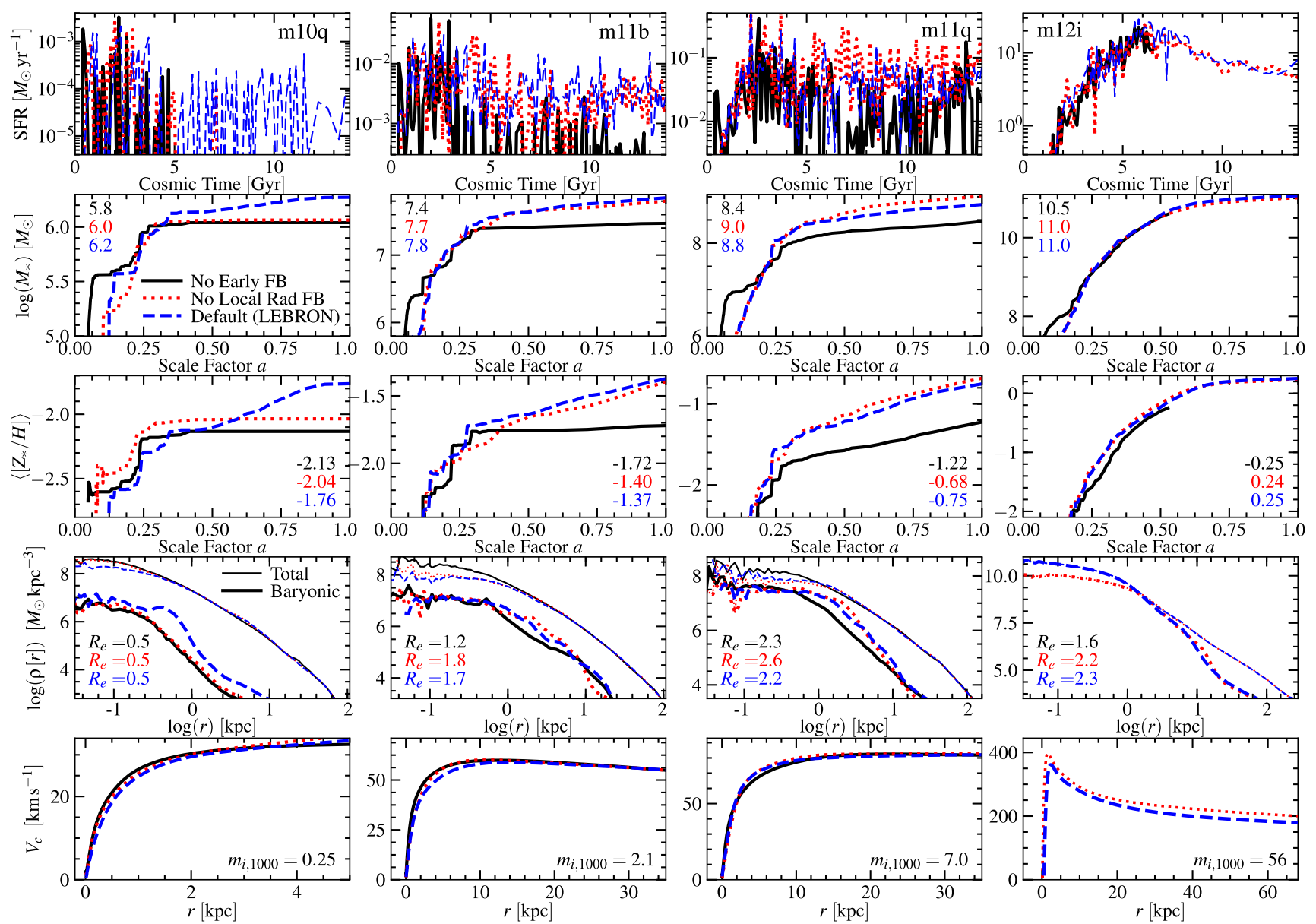

Figure 8. As Fig. 1, but comparing the effects of removing all 'early' FB (local radiative FB and 'early' fast O/B winds from massive stars), keeping the UVB in place. Some of the effects of local radiative FB can be 'made up for' by fast stellar winds (e.g. the small differences between 'Default' and 'No Local Rad FB' in m11q), as implemented in FIRE-2 (see the text for discussion). Removing both produces strong 'self-quenching' in both $\mathbf{m} 10 \mathbf{q}$ and $\mathbf{m 1 1 b}$, and nearly in m11q (up to LMC mass scales) - these galaxies overshoot (form many more stars early, at high redshifts), then blow out much of their baryonic gas mass. This leads to them having almost no late-time SF (despite being isolated dwarfs), and having strongly suppressed metallicities ( $\sim 0.3-0.5$ dex below the observed mass-metallicity relation). In $\mathbf{m 1 2 i}$ the differences in formation history are less obvious, but as shown below the galaxy has a wildly different morphology and is dominated by tiny, dense star clusters (the run has to be stopped at $z \sim 1$ ). Paper I and Paper II show that removing just stellar O/B winds, while keeping radiative FB, leads to much smaller effects than shown here - the important thing is that some early FB is present.

pressure, and O/B mass-loss, can 'compensate' to some extent for one another (Dijkstra \& Loeb 2009; Smith et al. 2017; Kimm et al. 2018, argue that multiply-scattered Ly $\alpha$ photons can also act in this manner, in metal-poor dwarfs). This should not be surprising: at solar metallicities the IMF-averaged momentum flux in massive stellar winds is $\sim L / c$, the same as that from singlescattering radiation pressure, and the momentum flux from warm gas pressure in a 'typical' massive H II region is also similar (see discussion in Lopez et al. 2011). They all act on similar (short) time and (small) spatial scales, as they come from the same massive stars. At low metallicities (e.g. our $\mathbf{m 1 0 q}$ dwarfs), stellar winds have a proportionally lower mass-loss rate and momentum flux, so they are less able to 'compensate' for a lack of radiative $\mathrm{FB}$, hence the stronger effects of removing local radiative FB in these runs.

Following the more detailed discussion in Grudić et al. (2018b), feedback from massive young stars (so $\left.L \sim\left(1200 L \odot / \mathrm{M}_{\odot}\right) M_{*}\right)$

does make the effects of removing radiation as well more dramatic in dwarfs, since the $\mathrm{O} / \mathrm{B}$ winds can 'make up for' less. imparts a momentum flux $\dot{p} \sim \eta L / c$ on gas in a typical GMC with surface density $M_{\mathrm{GMC}} / \pi R_{\mathrm{GMC}}^{2} \sim 100 \mathrm{M}_{\odot} \mathrm{pc}^{-2}$. Equating this to the gravitational force $\left(\sim G M_{\mathrm{GMC}}^{2} / R_{\mathrm{GMC}}^{2}\right)$ implies that the cloud will be destroyed when $M_{*} / M_{\mathrm{GMC}} \sim 0.05 / \eta$. So as long as a modest fraction of $L / c$ can couple, the cloud will self-regulate with $M_{*} / M_{\mathrm{GMC}} \ll 1$, producing an open-cluster type, unbound remnant, and re-cycling the mass, producing a low star formation efficiency locally. So while the difference between e.g. $\eta=1$ and $\eta=3$ (one versus all three mechanisms above acting in concert) might be detectable in individual cloud properties and star formation efficiencies (see e.g. Grudić et al. 2018c), all will produce effectively the same large-scale result. Given this, it is clear that strong constraints on which 'early FB' mechanisms dominate (under various conditions) will not come from galaxy-scale properties, but from observations which can probe these small-scale phenomena (consistent with many previous studies that have found galaxyscale star formation efficiencies are de-coupled from cloud-scale star formation efficiencies; see e.g. Hopkins et al. 2011, 2013; Federrath \& Klessen 2012; Agertz et al. 2013; Orr et al. 2018; Orr, Hayward \& Hopkins 2018; Semenov, Kravtsov \& Gnedin 2018). 

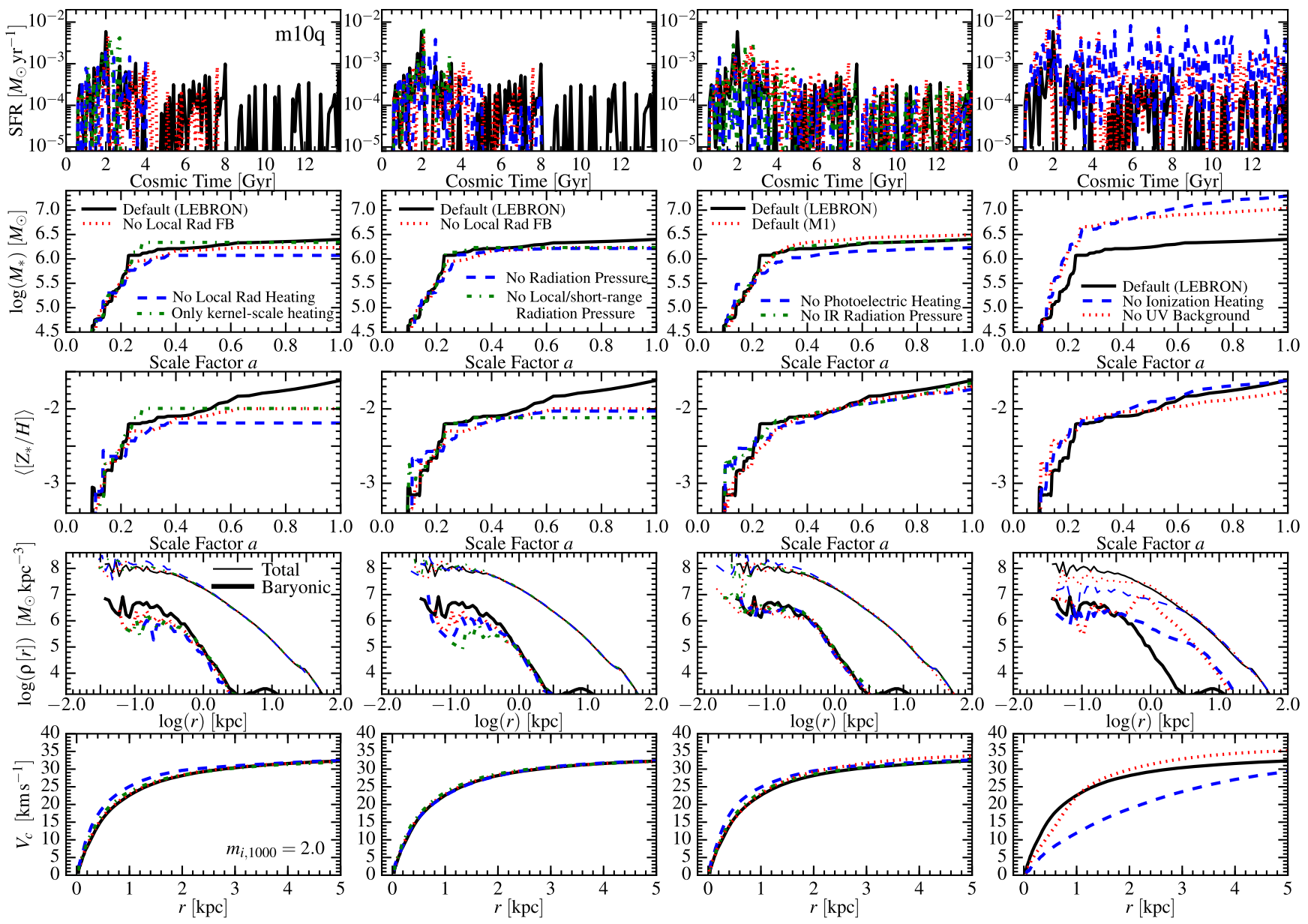

Figure 9. Effects of individual radiative FB channels/algorithmic aspects, using our Default (LEBRON) scheme, in m10q, as Fig. 1, at one level lower resolution. All panels compare our Default (LEBRON) runs to variations. (1) 'No Local Rad FB,' we see without local radiative FB the early-time SFH is again overly bursty ('gentler' radiative FB fails to slow down SF before $\mathrm{SNe}$ ), leading to more violent 'blowout' of metals and gas, suppressing [Z/H] and the $z=0$ gas fraction. (2) 'No Local Rad Heating': we turn-off all local (non-UVB) radiative heating terms (Compton, photoionization, photoelectric, from simulation stars) but keep radiation pressure; results are similar to 'No Local Rad FB.' (3) 'Only kernel-scale heating' only allows local radiative heating in gas which is an immediate neighbour of a star particle (keeping UVB and radiation pressure); effects are similar to 'No Local Rad Heating.' Long-range photoheating appears to be sub-dominant. (4) 'No Radiation Pressure' turns off all RP; results are similar to 'No Local Rad FB.' (5) 'No local/short-range radiation pressure' turns off the 'short-range' (kernel-scale) RP terms, but keeps long-range RP; the effects are similar to removing all RP (dynamical RP effects mostly occur on small scales). (6) 'Default (M1)' uses M1 instead of LEBRON, results are similar to Default (LEBRON). (7) 'No Photoelectric Heating' turns off just photoelectric heating; effects are weak. Photoionization is the most important heating term. (8) 'No IR Radiation Pressure' ignores IR re-radiation and multiple-scattering. Effects are negligible in low-metallicity dwarfs. (9) 'No Ionization Heating' turns off local and UVB-based photoionization heating; effects are similar to 'No Radiative FB' and slightly stronger than 'No UV Background.' (10) 'No UV Background' keeps all local radiative FB, but disables the UVB; effects are similar to 'No Ionization Heating.' Although the UVB clearly has the most dramatic effects on dwarfs, local warm gas pressure from photoionization heating and single-scattering RP are both significant.

\subsection{Effects of different radiative feedback channels}

Figs 9-11 break down the effects of individual radiative feedback channels, in turn. In Fig. 9, we take the 'Default (LEBRON)' algorithm as a base, then turn-off individual components of radiative feedback in turn to examine their separate effects. Owing to computational expense, we focus on comparison of one cosmological simulation ( $\mathbf{m 1 0 q}$ ) where the total effect of radiative FB is significant, and run these tests at one level lower resolution than our initial comparison in Figs 1 and 2 (but note that the behaviours in all four variations 'Default (LEBRON),' 'Default (M1),' 'No Local Rad FB,' and 'No Radiative FB' are essentially identical at this and the higher resolution level). Fig. 10 begins from our 'Default (M1)' model and similarly turns off, in turn, different wavebands evolved here. Fig. 11 repeats the exercise from Fig. 9 in an MW-mass system, re-starting our $\mathbf{m 1 2 i}$ simulation at redshift $z \approx 0.05$ and re-running it to $z=0$ (approximately $\sim 1 \mathrm{Gyr}$ ) as in Paper I. The advantage of the 'controlled restart' is that it allows us to see the effects of different feedback specifically in high-mass galaxies: since the MWmass system 'begins' as a dwarf (at high redshift), effects there resemble our $\mathbf{m 1 0 q}$ run, and propagate forward (confusing the comparison).

Fig. 12 compares our dwarf simulations, with our 'Default (M1)' implementation (five-band RHD including ionizing/EUV, photoelectric/FUV, NUV, optical/NIR, and MIR/FIR radiation) compared to the 'Extended Network (M1)' described in Section 3.2.1 and Appendix A, which expands this to a 10-band RHD treatment 

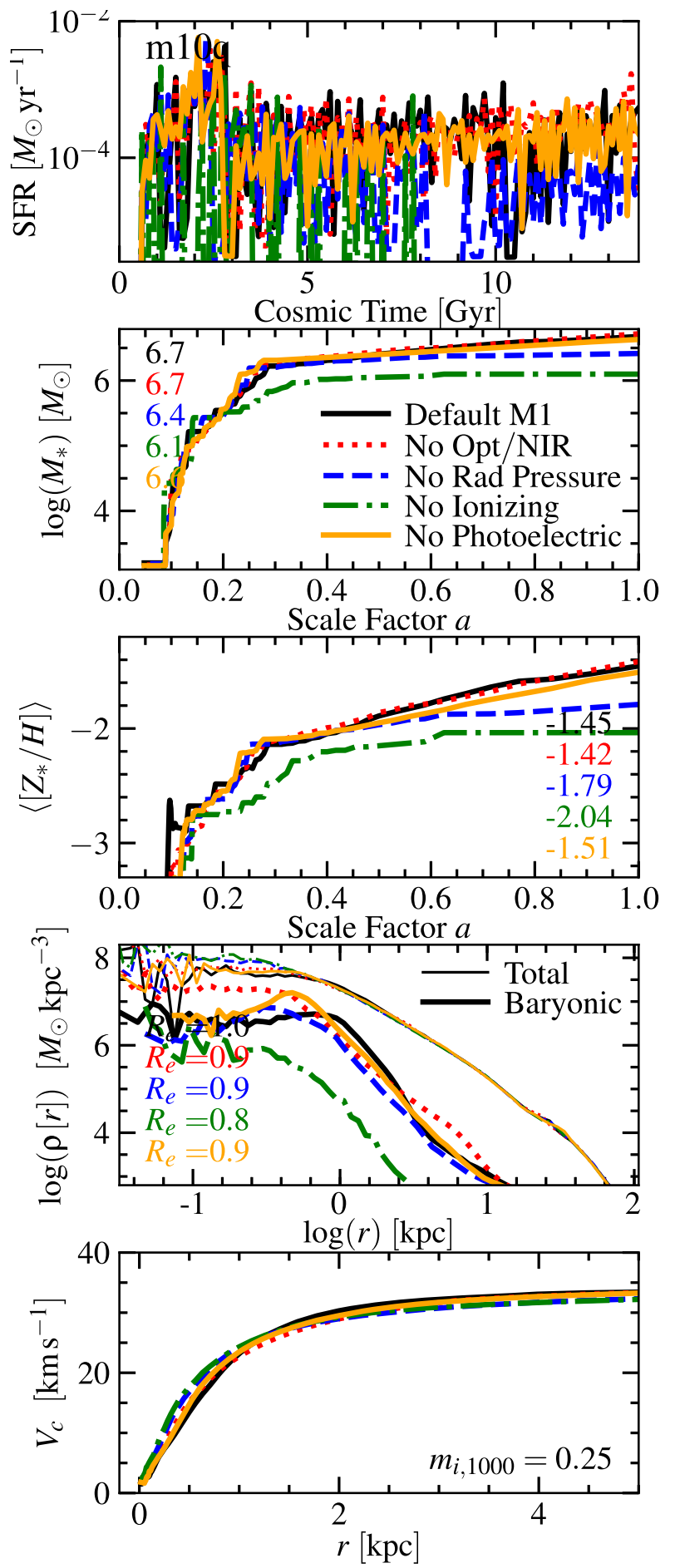

Figure 10. Effects of different local radiative FB channels, as Fig. 9, in our dwarf $\mathbf{m 1 0 q}$, but for runs using the M1 photon transport algorithm (turning off different wavebands in turn). Consistent with Fig. 9, singlescattering radiation pressure has a modest effect (with photoheating present). Removing all local ionizing photons (both their heating and radiation pressure), but keeping the UVB, is similar to removing all local radiative FB. Other bands have weaker effects at dwarf masses.
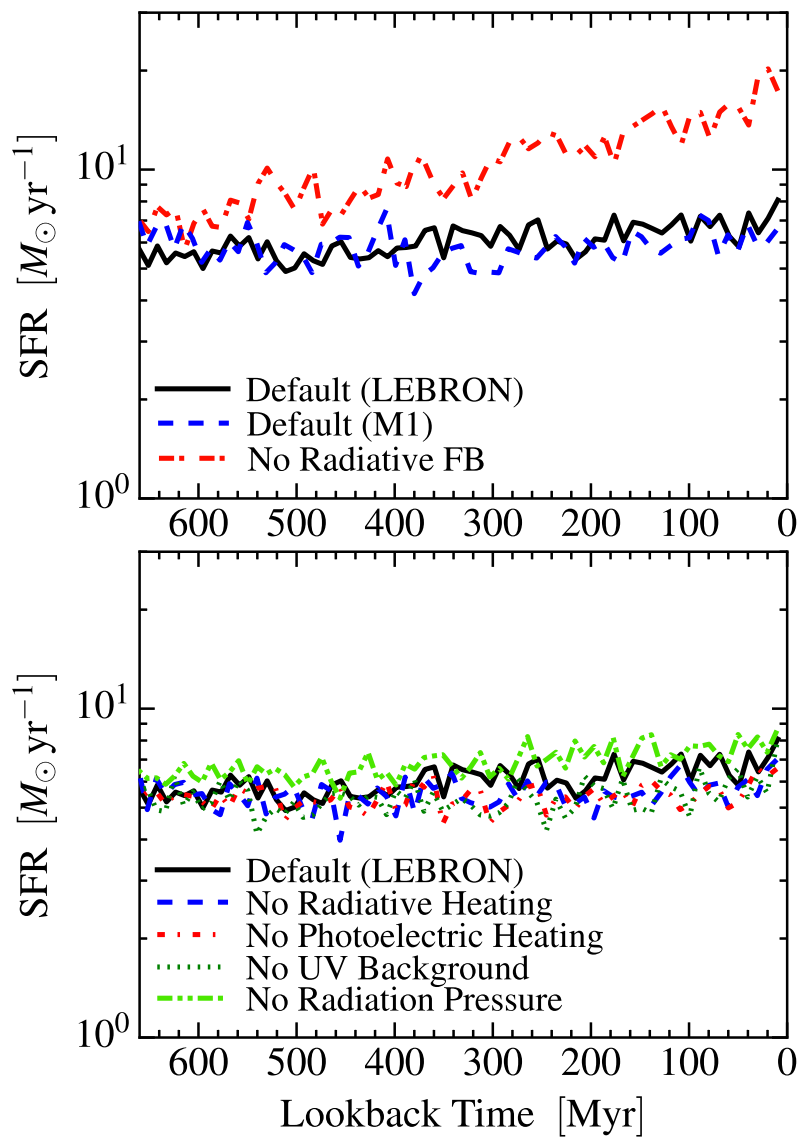

Figure 11. Variations of the radiative feedback, as Fig. 9, but re-starting a run of $\mathbf{m 1 2 i}$ from identical ICs at $z \approx 0.05$, and running it to $z=0$ with varied physics. Removing radiative FB leads to a significantly higher SFR at $z \sim 0$, for fixed initial conditions (e.g. galaxy gas mass) - the apparently smaller effect in Fig. 2 is because the 'No Radiative FB' run exhausts somewhat more gas earlier, changing the late-time galaxy properties. LEBRON \& M1 give similar results. Removing either RP or radiative heating alone produces little effect, but removing both together has a large effect - one can 'make up for' the other (along with stellar O/B mass-loss).

(dividing the ionizing band into four sub-bands with separate $\mathrm{HeI}$ and HeII ionizing bands, and adding Lyman-Werner, soft and hard X-rays, and dynamical radiation temperature-dependent FIR bands).

\subsubsection{The metagalactic UV background}

As shown above, for dwarfs, the most important form of radiative FB is the UVB: e.g. turning off all photoheating in $\mathbf{~ m 1 0 q}$ results in order-of-magnitude larger mass, while keeping just the local stellar radiation but removing the UVB produces factor of $\sim 5$ larger stellar mass (fourth column of Fig. 9). At $z=0$, assuming a continuous SFR $\dot{M}_{*}$, the same stellar SED templates used in-code, optically thin photon escape, and that SF is concentrated near a galaxy centre ( $r=0$, so flux scales $\propto 1 / r^{2}$ ), the UVB (also taking the in-code values) should dominate the UV radiation energy density emitted by the young stars at distances $r \gtrsim 2 \mathrm{kpc}\left(\dot{M}_{*} / 10^{-3} \mathrm{M}_{\odot} \mathrm{yr}^{-1}\right)^{1 / 2}$, so this is not surprising.

Somewhat less obviously, but consistent with previous studies (e.g. Thoul \& Weinberg 1996), we see significant effects from the 

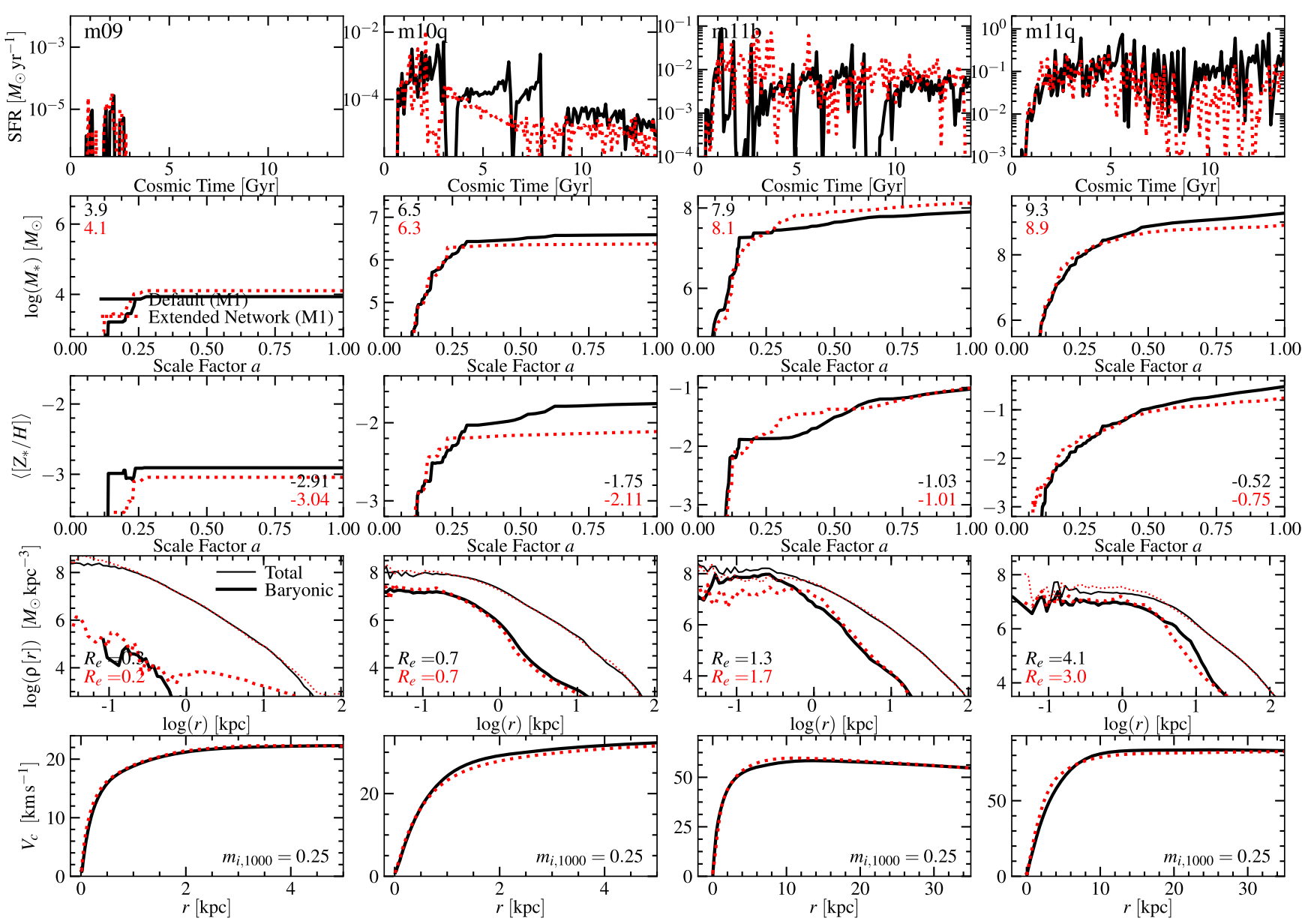

Figure 12. Effects of extended, additional radiative feedback channels on the simulations, as Fig. 10. We compare 'Default (M1)' and runs using the 'Extended Network' in M1, described in Section 4.3.5. The latter includes all the identical 'Default (M1)' physics, but adds several bands including: (1) separate soft and hard X-rays from X-ray binaries, and associated Compton heating, (2) dividing our single-band ionizing spectrum (which assumes a universal spectral shape for ionizing photons) into four separate sub-bands (separately tracking He I and He II ionizing photons), (3) treating the IR not as a grey, single-band 'bin' but self-consistently evolving the dust and IR radiation temperature fields and using a self-consistent opacity, with coupled dust-gas thermal heating and exchange, and (4) adding explicit Lyman-Werner band transport with an approximate treatment of its effects on molecular cooling. These are all expected to have small effects at this mass and redshift range, and we confirm this (the effects on the SFRs are largely consistent with stochastic run-to-run variations).

UVB extending to $V_{\max }$ as large as $\sim 100 \mathrm{~km} \mathrm{~s}^{-1}$. In haloes with $M_{\text {vir }} \sim 10^{11} M_{\odot}$ and $V_{\max } \sim 50-100 \mathrm{~km} \mathrm{~s}^{-1}$ (m11b and $\left.\mathbf{m 1 1 q}\right)$, the UVB suppresses the $z=0$ stellar mass by factors $\sim 2-3$, and in haloes with $V_{\max } \sim 40-50 \mathrm{~km} \mathrm{~s}^{-1}\left(M_{\text {vir }} \sim 10^{10} M_{\odot}\right.$, our $\mathbf{m 1 0 q}$ ) the difference is order-of-magnitude (Figs 1 and 2). ${ }^{11}$ These are well above the classical UVB 'quenching' threshold $\left(V_{\max } \sim 10-20 \mathrm{~km} \mathrm{~s}^{-1}\right)$, and indeed are not 'quenched' with a UVB. The calculation above shows that the UV radiation energy density from local stars dominates inside the galaxy effective radii (compare $R_{e}$ and $\dot{M}_{*}$ to the equation above), so this is not where the UVB has an effect. However, at radii $\gtrsim R_{\text {vir }}$ at $z \sim 0$, the circular velocities are $\sim 20-50 \mathrm{~km} \mathrm{~s}^{-1}$ in these more massive haloes, so the pressure support from the UVB contributes substantially, and suppresses the baryonic mass inside of $R_{\mathrm{vir}}$ by factors as large as $\sim 5-10$ (directly visible in the baryonic mass profiles; Figs 1 and 2).

\footnotetext{
${ }^{11}$ Also as noted in Section 4.1, because of the effects of stellar feedback on dark matter core creation, the circular velocity itself can be non-linearly sensitive to the change in star formation efficiency, while other quantities like metallicity change as expected with stellar mass.
}

Thus, we confirm that the UVB provides an important 'preventive' or 'suppressive' form of FB up to $V_{\max } \sim 50-100 \mathrm{~km} \mathrm{~s}^{-1}$.

By MW mass $\left(V_{\max } \gtrsim 200 \mathrm{~km} \mathrm{~s}^{-1}\right)$, the UVB has almost no effect on the primary galaxy, as expected. Of course, from the arguments above, we expect it to have a large effect on the mass function of satellites (small dwarfs) around the Local Group. This will be investigated in more detail in future studies (Wheeler et al., in preparation) which examine more realistic allowed variations in the UVB and their effect on satellite properties.

\subsubsection{Local photoionization heating}

Fig. 9 shows that turning off either local-photoionization heating, or radiation pressure, within the LEBRON scheme, produces a similar effect to turning off all local radiative feedback. We have also run a parallel set of M1 runs to Fig. 9, presented in Fig. 10. With the M1 runs in Fig. 10 we see that removing all local ionizing/UV photons (i.e. both their heating and radiation pressure) produces effects similar to removing all local radiative $\mathrm{FB}$, while removing just the radiation pressure (keeping photoheating) produces a significant, but not-as dramatic effect. The two schemes (LEBRON and M1, in 


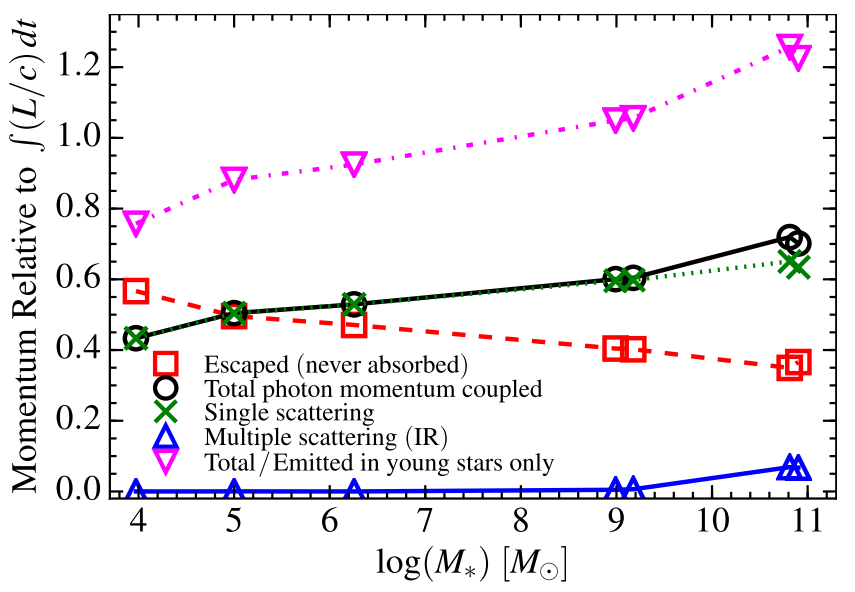

Figure 13. Diagnostics of photon absorption. We study a subset of our highest-resolution 'Default (LEBRON)' simulations from Table 1 and Paper I: m09, m10v, m10q, m11q, m11v, m12i, m12f (points, left-to-right). We compare the $z=0$ stellar mass of the primary galaxy, and total luminosity/momentum coupled to gas via various channels (labelled), integrated over all cosmic time (including all simulation stars, but this is dominated by the primary galaxy). We compare this to the total produced ( $E=\int L \mathrm{~d} t$, or momentum $=E / c$ ). The fraction coupled rises slowly from $\sim 0.4$ in ultrafaints to $\sim 0.8$ in $\mathrm{MW}$-mass galaxies. The dust opacity (proportional to $\mathrm{Z} / \mathrm{Z}_{\odot}$ ) is much larger at MW mass, but most of the ionizing continuum is absorbed by neutral gas in dwarfs (the absorbed fraction is closer to $\sim 100$ per cent of the light emitted by young, hot stars $<50$ Myr old). Roughly $\sim 1 / 2$ of emitted radiation escapes without absorption, primarily optical/NIR from older ( $\approx 100 \mathrm{Myr}$ ) populations. Single-scattering dominates: resolved multiplescattering in the IR (the only IR term included in the FIRE simulations) accounts for just $\sim 0.1 \mathrm{E} / \mathrm{c}$ in the most massive systems, and much less in dwarfs (with lower dust-to-gas ratios).

Figs 9 and 10, respectively) are therefore qualitatively consistent. In both schemes, both local photoionization heating and radiation pressure are important for the effects we described above.

Interestingly, in Fig. 11 at MW masses we see the opposite: turning off photoionization heating alone or radiation pressure alone produces almost no effect, but turning off both at the same time produces a large effect on the SFR. It appears that in $\mathbf{m 1 2 i}$, photoheating and radiation pressure are able to more directly 'take over' from one another (either one can pre-process large GMCs, such that the effects of SNe exploding in those clouds, for example, is similar). This is similar to our conclusions in FIRE-1 (Hopkins et al. 2014), although the overall effect was stronger there owing to the different treatment of $\mathrm{O} / \mathrm{B}$ mass-loss, as discussed above. This is also consistent with previous studies of non-cosmological, isolated galactic discs, including full RHD treatment of photoionizing stellar feedback (Kannan et al. 2014b; Rosdahl et al. 2015; Emerick et al. 2018).

Why are radiation pressure and photoionization (as well as other 'early feedback' mechanisms discussed above) able to 'take over' or 'compensate' for one another in MW-mass systems more efficiently than in dwarfs? This is at least partly a combination of metallicity and density effects. At solar metallicities (e.g. typical MW-like conditions), the optical depth to NIR/optical/NUV photons through GMCs is appreciable ( $\tau \gtrsim 1)$, so single-scattering radiation pressure carries $\sim L / c$ momentum flux, while at low metallicities, the dust opacity is small so only ionizing photons are efficiently absorbed - thus the radiation pressure forces are suppressed by a factor $\sim 2$ (see Fig. 13 and Section 4.4 below). This is similar to the effect of metallicity on the momentum flux in early $(\mathrm{O} / \mathrm{B})$ stellar winds (discussed in Section 4.2). Meanwhile, for $Q \sim 1$ discs, the typical velocity dispersions in large GMC complexes scale with the dispersion in the disc (see e.g. Hopkins et al. 2012b); so in MW-mass systems, $\mathrm{H}$ II regions expanding at the ionized gas sound speed $\sim 8-10 \mathrm{~km} \mathrm{~s}^{-1}$ are only marginally able to unbind clouds with similar dispersions, while in dwarfs the lower densities and cloud internal velocity dispersions allow them to be more easily un-bound (for a detailed demonstration of this in cloud simulations, see Grudić et al. 2018b).

In our LEBRON RHD method, we can also control separately the short-range (kernel-scale surrounding each star) and longrange (propagated via the gravity tree) components of the radiative feedback. For photoionization heating, Fig. 9 demonstrates that the long-range component is actually the most important in our dwarf galaxy. In other words, heating diffuse gas (e.g. the extended gas discs which form when there are inflows, and diffuse gas within the halo/CGM surrounding the galaxy) is physically most important for the slowing/regulating star formation we see in the dwarf galaxies. The short-range term primarily manifests as local $\mathrm{H}$ II regions around the youngest star particles - these help destroy GMCs before SNe explode, so have some significant effects, but (because the gas is dense) involve proportionally little gas mass. In contrast, photoheating a diffuse disc to $\sim 10^{4} \mathrm{~K}$ in a galaxy with $V_{c} \sim 30 \mathrm{~km} \mathrm{~s}^{-1}$ raises the local Toomre $Q \gg 1$, suppressing star formation significantly (for a more detailed study using ray-tracing in comparison to 'local' ionization treatments in non-cosmological simulations, see Emerick et al. 2018).

\subsubsection{Photoelectric heating}

At both dwarf and MW masses, we see weak galaxy-scale effects from photoelectric heating. This is consistent with nearly all previous studies of (non-cosmological) galaxy and star formation simulations (see e.g. Dobbs et al. 2008; Tasker \& Bryan 2008; Tasker 2011; Hopkins et al. 2012b; Hu et al. 2016, 2017; Richings \& Schaye 2016; Su et al. 2017). Essentially all of these studies (some of which reach mass resolution $\sim 1 \mathrm{M}_{\odot}$ ) conclude that while the details of cold gas phase structure $\left(\ll 10^{4} \mathrm{~K}\right)$ and fragmentation down to stellar mass scales (i.e. the scales where the thermal Jeans mass in GMCs and cold gas becomes relevant) are sensitive to photoelectric heating (and indeed we do see effects in the temperature-density distribution in Figs 4 and 5), the behaviour we resolve in our studies here is all well in the regime where supersonic turbulence dominates the dynamics. Given that Paper I showed there were very weak effects on most properties studied here if one simply turned off all cooling below $\sim 10^{4} \mathrm{~K}$, and that perhaps the most important effects of radiation in dense/cold gas gas (namely regulating the IMF; see Offner et al. 2009, 2014; Bate 2012; Hansen et al. 2012; Guszejnov et al. 2016, 2017) are implicitly sidestepped by assuming a fixed stellar IMF, it should not be surprising that explicit treatment of photoelectric heating is a generally subdominant effect here.

\subsubsection{Single-scattering radiation pressure (UV/Optical/NIR)}

As discussed above in Section 4.3.2, in our dwarf $\mathbf{m 1 0 q}$, both radiative heating and radiation pressure produce similar effects, and the two in concert produce a smoother (less bursty) star formation history that continues down to $z=0$ (Figs 9 and 10). Fig. 9 does confirm that the effects of radiation pressure are dominated by the local/short-range (i.e. kernel-scale) coupling: specifically, we disable the momentum coupling from the locally absorbed photons 
in the LEBRON method (those absorbed within the 'short-range' component calculated within a single kernel around the stellar source, as described in Paper I), but retain the radiation pressure from the long-range component propagated through the gravity tree, and find this is very similar to simply disabling all radiation pressure. This is expected, given the arguments below, since most of the coupling occurs around young stars embedded in GMCs. But it is also reassuring: in our default RHD method, only the longrange component is non-photon-conserving - this and the agreement between our default method and the M1 method suggest this is not a significant source of error. ${ }^{12}$ In our MW-mass $\mathbf{m 1 2} \mathbf{i}$ run in Fig. 11, it seems radiative feedback is important in regulating the SFR into better agreement with the Schmidt-Kennicutt law ${ }^{13}$ (similar to our conclusions in Hopkins et al. 2011), but in the absence of just radiation pressure, a similar effect can be 'made up for' by radiative heating. Although not shown, we again find the radiation pressure terms are dominated by local coupling.

The radiation pressure effects we see in Figs 9-11 are almost entirely single-scattering effects. To see this, Fig. 13 quantifies the total radiation pressure which has coupled to gas in the galaxy, in a subset of our simulations (using our LEBRON scheme). Specifically, we record the total momentum imparted from photons to gas $p_{\text {coupled }} \equiv \sum\left|\Delta \mathbf{p}_{a}\right|$ (with the sum over all particles and time-steps in the simulations, every time a radiation pressure term is calculated), and compare this to the integrated photon momentum from all photons emitted by all stars in the simulation, $p_{\text {available }} \equiv c^{-1} \sum L_{\text {bol }}^{a}\left(t-t_{\text {form }}\right) \Delta t_{a}$ (the sum over the bolometric luminosity of all star particles, integrated over all times, since each particle forms). We define $\langle\tilde{\tau}\rangle \equiv p_{\text {coupled }} / p_{\text {available, and see this is }}$ typically $\sim 0.5-0.7$, i.e. slightly less than complete single-scattering. As discussed below, that is because some of the emitted optical from older stars escapes, while most of the UV/ionizing radiation is absorbed.

If we directly quantify the multiply-scattered component (here, the radiation pressure from the IR bands), we see it is totally negligible in dwarfs, and rises to just $p_{\text {multiple }} \sim 0.1 p_{\text {available }}$ in MWmass galaxies. This can be important for e.g. the dusty nuclei of massive galaxies (during bursts of star formation) and/or individual star cluster formation episodes; but it is not a dominant effect for most star formation.

\subsubsection{Additional channels}

With the extended frequency network active (Fig. 12), we see relatively little systematic change in our dwarf galaxies. Some differences in the detailed SFR versus time are clearly evident, along with $\sim 10-20$ per cent changes in mass, but these appear to be essentially random, and are consistent with stochastic run-torun variations in these simulations (see Su et al. 2017). This is not particularly surprising, because the additional mechanisms in this network are not expected to dominate the channels included in our

\footnotetext{
${ }^{12}$ In previous work, e.g. Hopkins et al. (2012a), we argued that the longrange component might be key to re-accelerating or 'lofting' winds, in particular in massive starbursts, but we did not explicitly attempt to separate the various components as we do here. While this certainly might still occur, our results here suggest this is not critical for most of the outflows in dwarfs, which are predominantly driven by $\mathrm{SNe}$ explosions.

${ }^{13}$ Specifically, the 'Default' run in Fig. 11 agrees well with the observed Schmidt-Kennicutt relation, as shown in Orr et al. (2018), while the 'No Radiative FB' run has the same gas surface density (by construction in these restarts, but a factor $\sim 3-4 \times$ larger SFR.
}

'default' simulations, but we briefly discuss these channels in turn to note why this is.

(i) Compton heating (via XRBs): If we assume a constant SFR $\dot{M}_{*}$, and use this to estimate the soft and hard X-ray luminosity produced by XRBs (Appendix A), and in turn the Compton heating rate $Q_{\text {Compton }}$ (see Paper I) for gas with density $n$ at a distance $r$ from the galaxy, and compare this to the normal cooling rate $\left(\dot{e}_{\text {cool }}=\Lambda n^{2}\right.$ with $\left.\Lambda \sim 10^{-23} \mathrm{erg} \mathrm{cm}^{3} \mathrm{~s}^{-1}\right)$, we obtain $Q_{\text {Compton }} / \dot{e}_{\text {cool }} \sim$ $10^{-8}\left(\dot{M}_{*} / \mathrm{M}_{\odot} \mathrm{yr}^{-1}\right)\left(n / 0.01 \mathrm{~cm}^{-3}\right)^{-1}(r / 10 \mathrm{kpc})^{-2}$. In other words, Compton heating is totally negligible. ${ }^{14}$ This could be important in the near vicinity $(r<1 \mathrm{pc})$ of a luminous AGN, but should not be important from stars, and we confirm this by including these terms in our extended network. A more interesting $\mathrm{X}$-ray feedback channel is either non-equilibrium metal-line overionization (altering the metal-line cooling rate) or IGM ionization by redshifted X-rays, but our simulations do not include the relevant physics or scales to follow these.

(ii) (Explicit) He ionization: Our extended network includes a four-band treatment for photoionizing radiation, with separate tracking of HeI and HeII (versus HI) ionizing photons. However, at the coarse-grained level here, we find this does not make a large difference to the bulk galaxy properties we see. Recall, the effects of ionization from local stars alone (if we still include a fixed UVB, radiation pressure, and O/B mass-loss) are relatively subtle, so it is not surprising that making our 'Default (M1)' ionization treatment slightly more accurate has small effects. Note that in our default treatment, we still account separately for $\mathrm{He}$ I and $\mathrm{He}$ II ionization in the cooling routines and chemistry calculations (see Paper I for details); we simply assume a spatially uniform spectral shape for the ionizing photons (fixed to the UVB spectral shape from FaucherGiguère et al. 2009, which can evolve in time). The spatial variations occur only on small spatial and time-scales in the vicinity of very young, hot stellar populations - these could easily be important to dynamics in individual $\mathrm{H}$ II regions and corresponding emission-line diagnostics, but are second-order on galactic scales.

(iii) Ly-Werner radiation: The extended network also includes a very approximate treatment of the effect of $\mathrm{H}_{2}$ dissociating (Lyman-Werner or LW) radiation on molecular cooling (specifically calculating an equilibrium molecular fraction depending on the incident flux, and reducing the metal-free contribution to the cooling rate below $<10^{4} \mathrm{~K}$ accordingly). However, as we showed and discussed at length in Paper I, completely removing this plus all metal-line and atomic cooling below a few thousand Kelvin effectively has no influence on our large-scale conclusions. Many other chemical studies have reached the same conclusions, specifically that molecular cooling produces essentially no appreciable dynamical effects on star or galaxy formation above metallicities $[\mathrm{Z} / \mathrm{H}] \gtrsim-3$ (or -5 , if dust cooling is included; see Glover \& Clark 2012; Hopkins et al. 2012b; Dopcke et al. 2013; Ji, Frebel \& Bromm 2014; Corbett Moran et al. 2018). Recently, Lupi et al. (2018) used the M1 implementation in GIZMO, and broadly similar stellar feedback models, coupled to the non-equilibrium KROME chemistry module (Grassi et al. 2014), to explore the nonequilibrium effects of $\mathrm{H}_{2}$ dissociating radiation on evolved galaxies in idealized and cosmological simulations. While they concluded the LW transport is, of course, important for correctly modelling the

\footnotetext{
${ }^{14}$ Cantalupo (2010) similarly show that the effect of XRBs on the cooling rates of CGM gas via indirect feedback (altering the ionization of the gas) is small unless the SFRs are extremely large $\left(\gg 100 \mathrm{M}_{\odot} \mathrm{yr}^{-1}\right)$ and the gas lies in a narrow range of temperatures around $\approx 1 \times 10^{5} \mathrm{~K}$.
} 
$\mathrm{H}_{2}$ and therefore associated diagnostics, it again had no significant dynamical effects on SFRs or other galaxy properties, in the mass and redshift range studied here.

(iv) IR thermal dust heating: Our extended network allows for the dust temperature to come into appropriate equilibrium with the IR radiation field, and thermally couple to the gas via dustgas collisions. This tends to raise or lower the gas temperature to be in equilibrium with the dust temperature around temperatures below $\sim 30-100 \mathrm{~K}$, above densities $\gtrsim 10^{6} \mathrm{~cm}^{-3}$ (where it becomes dominant). Unsurprisingly, for the reasons above, and because these densities are well above those resolved here, this has negligible effects here. As discussed above, this might in nature determine the IMF turnover (regulating the thermal Jeans mass around $\gtrsim 0.1 \mathrm{M}_{\odot}$ ), but we assume an IMF and do not resolve this, and confirm it has no large-scale direct dynamical effects (though of course if the IMF changed, this could be important for feedback).

(v) Non-grey IR: In the extended network, the IR is no longer treated as a single bin with a single opacity, but rather as a pseudo blackbody where the radiation, dust, and gas temperatures are all evolved independently and explicitly, with opacities that depend on these temperatures (and metallicity). It has been argued that this more sophisticated treatment could substantially alter the ability of IR radiation to multiply-scatter (because it will be downgraded to longer wavelengths and lower opacities as it does so). However, we show below (Section 4.3.6) that such multiplescattering accounts for a very small fraction of the total radiative FB, so (unsurprisingly) these higher order corrections to it make little galaxy-scale difference.

(vi) Ly $\alpha$ resonant scattering: Our RHD methods do not allow us to consider multiple Ly $\alpha$ scattering. However, we briefly discuss it here. Recently, Kimm et al. (2018) considered a detailed study of simulations including a sub-grid model for Ly $\alpha$ RHD (together with a similar multiband treatment of ionizing, photoelectric, single, and multiple scattering in UV/optical/IR to our 'Default (M1)' runs) in idealized (non-cosmological) simulations of a dwarf galaxy (similar in mass to our $\mathbf{m 1 1 b}$ ). Although the details of their numerical hydrodynamic method, treatment of SNe and stellar mass-loss differ substantially, they reach remarkably similar conclusions to our study here about the role of radiation and early feedback in dwarf galaxies. In particular, they argue that although the Ly $\alpha$ luminosity is only a small fraction of the continuum, it can be multiply-scattered giving a net momentum flux $\dot{p}_{\mathrm{Ly} \alpha} \sim(10-300) L_{\mathrm{Ly} \alpha} / c$, larger by several than the continuum. ${ }^{15}$ This amplifies all the effects studied here: star formation is less bursty, more warm gas is supported, and cluster formation is suppressed. Studies in more idealized galactic wind environments, but using explicit Ly $\alpha$ RHD, have reached qualitatively similar conclusions (see Smith et al. 2017, 2018).

\subsubsection{Infrared radiation and photon-trapping or multiple-scattering}

As shown in Figs 13 and 14, the momentum $L / c$ contributed by multiply-scattered IR radiation is completely negligible in dwarf galaxies (reaching values $\ll 0.01$ ), rising with galaxy mass until it reaches just $\sim 0.1$ in MW-mass galaxies. This rising importance is expected, given the increasing metallicities (hence dust opacities) and surface densities in more massive galaxies (e.g. m10q, with median gas surface density $\Sigma_{\text {gas }} \sim 10 \mathrm{M}_{\odot} \odot \mathrm{pc}^{-2}$ and metallicity

\footnotetext{
${ }^{15}$ As they note, this effect will likely diminish rapidly in more massive, dust-rich galaxies, as dust destroys Ly $\alpha$ photons.
}

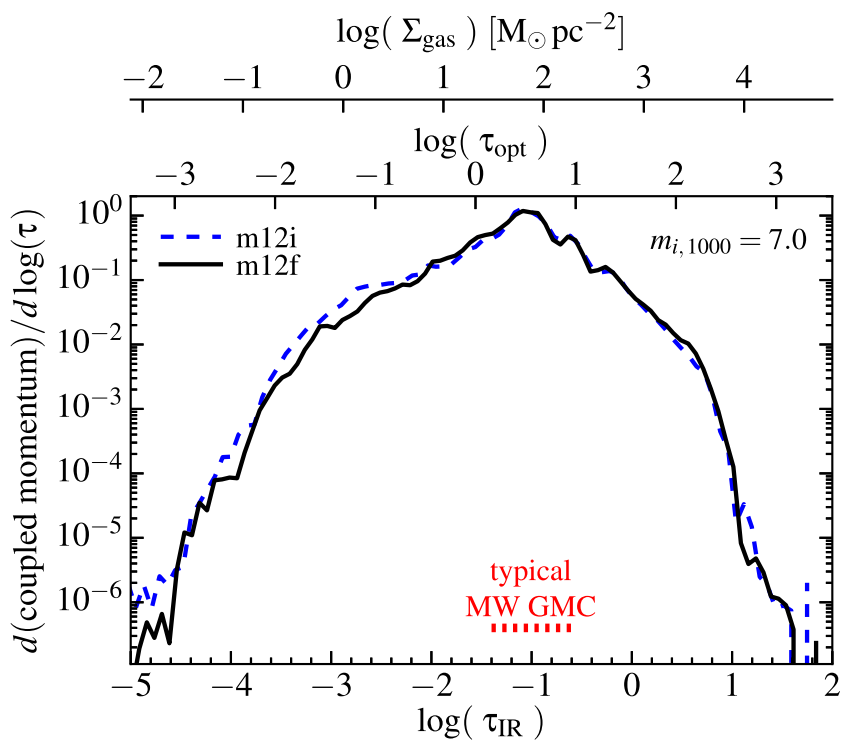

Figure 14. Distribution of column densities (and approximate corresponding IR and optical depths) at which photons are absorbed, weighted by fraction of total photon momentum coupled to gas. Each time photons are absorbed we estimate the local $\Sigma_{\text {gas }}$ from our Sobolev-type approximation and record it, for the highest-resolution $\left(m_{i, 1000}=7\right) \mathrm{MW}$-mass $(\mathbf{m 1 2 i}$, m12f) simulations with the 'Default (LEBRON)' FIRE treatment from Paper I. The distribution has a sharply peaked 'core' (with most of the absorption) with disperson of just $\sim 0.15$ dex around $\Sigma_{\text {gas }} \sim 100 \mathrm{M}_{\odot} \mathrm{pc}^{-2}\left(\tau_{\mathrm{IR}} \sim 0.1\right.$, $\tau_{\text {optical }} \sim$ couple $)$ - but broad tails of the form $d P / d \log \tau \propto \tau^{ \pm 1}$, similar to what is expected for a quasi-fractal or lognormal ISM density distribution (Hopkins et al. 2011). Note that just $\sim 5$ per cent $(\sim 0.01$ per cent) of the momentum comes from regions with resolved $\tau_{\mathrm{IR}}>1(>10)$. We compare the $\pm 1 \sigma$ range of surface densities through to the centre of a GMC in the MW (= $\left.(1 / 2) M_{\mathrm{GMC}} / \pi R_{\mathrm{GMC}}^{2}\right)$ from the observed compilation in Bolatto et al. (2008) - this is almost exactly the optical depth we see dominating absorption. In other words, most of radiative feedback comes from singlescattering/absorption from embedded stars in 'normal' GMCs.

$Z \sim 0.02 Z_{\odot}$, has median IR optical depth $\left.\sim 0.0004\right)$. As discussed below, even in MW-mass galaxies, most absorption occurs in 'typical' GMCs with IR optical depths $\sim 0.1$. Over the limited range of resolution we explicitly probe here (e.g. decreasing the mass resolution by a factor $\sim 8-64$ ), this conclusion appears robust, and (as noted above) our previous resolution studies (Hopkins et al. 2018b; Guszejnov et al. 2017, 2019) have shown that this characteristic $\Sigma_{\text {gas }}$ and (corresponding) IR optical depth is robust over factor $>100$ changes in resolution. However, the 'tail' of absorption at very high column densities in Fig. 14 is more prominent (as expected) at higher resolution where we can follow smaller, denser structures (clumps, cores) which typically have higher column densities.

We stress that there is no artificial large 'boost factor' or 'added optical depth' applied to radiative feedback in any of our simulations. IR photons, in principle, can be trapped and multiplyscatter: if one has a source of luminosity $L_{\mathrm{bol}}$, surrounded by a sphere of gas with flux-mean optical depth $\tau_{\text {single }}$ to initial singlescattering (optical/UV) and appropriately weighted $\tau_{\text {eff, IR }}$ to the re-emitted IR photons, then the momentum flux imparted to the gas is $\dot{p}=\tilde{\tau} L_{\text {bol }} / c$, with $\tilde{\tau}=\left(1-\exp \left(-\tau_{\text {single }}\right)\right)\left(1+\tau_{\text {eff, IR }}\right) .{ }^{16}$ This $\tilde{\tau}$

\footnotetext{
${ }^{16}$ To derive $\tilde{\tau}$, begin by noting that locally at some position $\mathbf{x}$, the acceleration/momentum flux from photons at frequency $v$ is exactly $\partial(\rho \mathbf{v}[\mathbf{x}])_{v} / \partial t=\rho(\mathbf{x}) \kappa_{v} \mathbf{F}_{v}(\mathbf{x}) / c$. If we integrate over both volume and
} 
term is sometimes referred to as a 'boost factor'; it ranges from $\approx \tau_{\text {single }} \ll 1$ in the optically thin limit to $\approx 1+\tau_{\text {eff, IR }} \gtrsim 1$ in the IR optically thick limit. In our simulations (FIRE-1 and FIRE-2), we explicitly calculate the radiative acceleration, based only on the local opacity and incident flux at every resolved gas element position. This means that our simulations will, if anything, tend to underestimate the true momentum flux from radiation pressure - i.e. it is likely that if we massively improved our resolution, we might see a more important role for IR multiple-scattering in the cold, dense ISM at densities well above our current star formation threshold (see the discussion in Rosdahl et al. 2015). For example, $\tau_{\text {eff, IR }} \gg 1$ should in reality occur on sufficiently small scales around individual protostellar cores, but since these are not resolved in our simulations this contribution is not included (only the explicitly resolved contributions to $\tau$ are accounted for). However, the lifetime of this deeply buried phase is short $\left(\ll 10^{6} \mathrm{yr}\right)$, so the expectation is that the integrated momentum 'missed' is therefore small.

Unfortunately some confusion on this point owes to our older (pre-FIRE) work, specifically in Hopkins et al. (2011), so we wish to clarify it here. The Hopkins et al. (2011) simulations pre-dated the FIRE and GIZMO codes by several years, were non-cosmological, did not include any feedback other than radiation pressure, and used a sub-grid model to treat radiation which was fundamentally numerically different from either the LEBRON or M1 RHD methods. In that particular study, $\tilde{\tau}$ was multiplied by arbitrary factors to explore its effects; however the conclusion was that for most typical dwarf, dwarf starburst, or MW-like galaxies $\tilde{\tau} \sim 1-2$ in a time-averaged sense (and even for an intentionally extreme dense starburst-nucleus model with disc surface density $>1000 \mathrm{M}_{\odot} \mathrm{pc}^{-2}$, $\tilde{\tau}$ did not exceed $\sim 5-10$ ). The confusion on this point largely owes to fig. 5 in Hopkins et al. (2011), where we showed that for the starburst disc, the instantaneous, momentum-coupling-weighted $\tau_{\text {eff, IR }}$ reached $\sim 30-50$ - but this is just the statement that, at the moment a star particle 'turns on,' the resolved surface densities of the star-forming cores reached $\Sigma_{\text {gas }} \sim 10^{4} \mathrm{M}_{\odot} \odot \mathrm{pc}^{-2}$ (similar to those observed). As we noted therein, such high $\Sigma_{\text {gas }}$ and $\tau_{\text {eff, IR }}$ means that the core is almost immediately turned into stars or disrupted (the gas is exhausted or pushed away on a dynamical time $\sim 10^{5} \mathrm{yr}$ ), such that the 'effective' $\tilde{\tau}$ (time-averaged) is an order of magnitude lower (for more detailed discussion, see e.g. Hopkins \& Grudic 2018; Grudić et al. 2018d, b). This is essentially identical to the conclusions in many subsequent radiative-transfer studies (e.g. Krumholz \& Thompson 2012; Kuiper et al. 2012; Davis et al. 2014; Tsang \& Milosavljević 2015).

\subsection{Where does radiation couple?}

As noted above, Fig. 13 plots the total photon momentum absorbed - essentially, the fraction of the galaxy-lifetime-integrated stellar

frequency to obtain the total radiative force (momentum flux), we obtain $\partial \mathbf{p} / \partial t=\int d^{3} \mathbf{x} \rho(\mathbf{x}) \mathbf{F}(\mathbf{x}) \kappa_{\mathrm{F}}(\mathbf{x})$, where $\mathbf{F}$ is the total flux and $\kappa_{\mathrm{F}}$ the fluxmean opacity at $\mathbf{x}$. If we simplify by assuming spherical symmetry, then we can trivially solve this integral and obtain $\partial \mathbf{p} / \partial t=\tau_{\text {eff, IR }} L_{\mathrm{IR}} / c \hat{\mathbf{r}}$, where $\tau_{\text {eff, IR }} \equiv \int \mathrm{d} r \rho(r) \kappa_{\mathrm{F}}(r)$ and $L_{\mathrm{IR}}$ is some central source luminosity (a similar expression can be written without the symmetry assumption, using an appropriately angle-weighted average $\tau_{\text {eff, IR }}$ ). The IR luminosity $L_{\mathrm{IR}}$ comes from single-scattered photons absorbed by dust, with $L_{\mathrm{IR}} \approx L_{\mathrm{abs}}=$ $\left(1-\exp \left(-\tau_{\text {single }}\right)\right) L_{\text {bol }}$ (where $\tau_{\text {single }}$ is the flux-mean opacity for the input spectrum), so including their momentum we have $\partial \mathbf{p} / \partial t=\tilde{\tau} L_{\mathrm{bol}} / c \hat{\mathbf{r}}$ where $\tilde{\tau} \equiv\left(1-\exp \left(-\tau_{\text {single }}\right)\right)\left(1+\tau_{\text {eff, IR }}\right)$. luminosity $L$ which is absorbed by gas and dust in the system, and (correspondingly) the fraction which escapes without absorption or scattering. We also plot this total relative to the light emitted (primarily in UV) by young stellar populations (this can be $>1$ if some light from 'old stars' is also absorbed), and the light re-emitted and then absorbed in the IR (i.e. effectively 'multiply scattered'). Fig. 14 shows the distribution of column densities, in our MWmass galaxies, at which the absorption occurs (in our 'Default (LEBRON)' simulations).

The effective 'coupled fraction' of the emitted bolometric luminosity, $\langle\tilde{\tau}\rangle \sim 0.5$, ranging from $\sim 0.4$ in the smallest dwarfs to $\sim 0.7$ in MW-mass systems. A fraction $\sim 0.6$ (dwarfs) to $\sim 0.4$ (MWmass systems) escapes without ever being absorbed. The order-unity coupled and escaped fractions are remarkably weakly dependent on galaxy mass. This is because a comparable fraction of the timeintegrated bolometric output, integrated over a Hubble time, comes from (1) ionizing luminosity from very young stars, almost all of which is absorbed (the opacities are extremely high, and do not require dust, and many of the stars are buried in large columns), and (2) longer wavelength (optical/NIR) from older ( $\gtrsim 50-100 \mathrm{Myr}$ ), less luminous populations (which given the lower opacities and low dust content in the galaxy outskirts, tend to escape). It is also the case that, because galaxies are supersonically turbulent, there is a broad distribution of column densities through the disc to a random star at any time - thus an order-unity fraction of sightlines are always optically thin in the optical. Indeed, if we compare the coupled photon momentum to that integrated over stellar populations only up to an age of $\sim 100 \mathrm{Myr}$, we obtain $p_{\text {coupled }} \sim p_{\text {available }}(t<100 \mathrm{Myr})$.

Fig. 14 examines further where absorption occurs. We focus on the MW-mass systems, as this is (i) where the best observational constraints exist, and (ii) the only case where any significant IR multiple-scattering or dust absorption occurs. Since we will use our 'Default (LEBRON)' simulations, we use the highest resolution versions available, namely those with $m_{i, 1000}=7$, with all properties shown in detail in Paper I. In the simulations, every time radiation (from stars in the simulation) is coupled to gas in the simulation via the LEBRON algorithm, we calculate a local Sobolev-type estimate of the column density seen by those photons (specifically, $\langle\Sigma\rangle \approx \rho[h+\rho /|\nabla \rho|]$, where $h=\left(m_{i} / \rho\right)^{1 / 3}$ is the local resolution element size, and $\nabla \rho$ is the density gradient, to account both for the column density within a single 'cell' and approximate it integrated out to infinity). We record this and the amount of luminosity $\Delta L$ absorbed (equivalently, the momentum $\Delta L / c$ deposited). We then construct the $z=0$ distribution of 'column densities' (or approximate optical depths at different wavelengths) weighted by the absorbed luminosity.

We clearly see that the majority of the coupled radiation pressure occurs around optical depths of order a couple in the optical, or $\tau_{\mathrm{IR}} \sim 0.1$ - which corresponds neatly to the typical surface density of GMCs both in our simulations (see e.g. Hopkins et al. 2012b) and observed (Bolatto et al. 2008). In other words, most of the imparted radiation pressure comes from single-scattering of light from massive, still-embedded stars. A few percent of the momentum comes from regions with $\tau_{\mathrm{IR}}>1$ - exactly consistent with the ratio of photon momentum from multiple-scattering to the total imparted (for MW-mass systems) in Fig. 13. Given our resolution, this does not come from protostellar cores (which are totally un-resolved), but from periods where the galactic nucleus (or massive 'clump complexes' at high redshift) experiences tidal compression and rapid gas inflow in a starburst on resolved scales of $\sim 100 \mathrm{pc}$. In these rare phases, the IR terms may dominate - and 
in future work we will explore how this does or does not matter for extreme starburst environments. But clearly, the multiple-scattering effects are minimal for most of the galactic star formation on the scales resolved here.

\subsection{Numerical methods}

Here we briefly summarize the impact of numerical RHD methods on the simulation results.

For validation tests of the numerical methods studied here (e.g. confirmation that the implementations recover the correct answer in the limits under which their fundamental assumptions are valid), we refer to several previous studies (e.g. Hopkins et al. 2012b, 2014, 2018b; Roth et al. 2012; Ma et al. 2015; Grudić et al. 2018b; Hopkins \& Grudic 2018; Lupi et al. 2018) as well as parallel studies using the same classes of methods in different codes (Rosdahl et al. 2013; Kannan et al. 2014b; Rosdahl et al. 2015; Rosdahl \& Teyssier 2015; $\mathrm{Hu}$ et al. 2016, 2017; Emerick et al. 2018). It is not our intention to repeat these studies here.

Rather, in the sections above, we considered the physical consequences of variations in the numerical methods. First, we note the variations of LEBRON or M1 which we have studied. The LEBRON method allows us to formally turn on or off different physical components of the radiative FB: we can specifically disable local (kernel-scale) or long-range (tree-based) radiation pressure and/or photo-heating terms, or we can remove the 'local extinction' operation which attenuates the spectrum before propagation to longrange distances. These variations are compared in Fig. 9, and have been discussed above: specifically they allow us to show that most of the RP comes from absorption in the vicinity of stars (consistent with the study of where radiation is absorbed, also above), and that local extinction of non-ionizing radiation is un-important in small dwarfs where the optical depths are relatively small (but important in massive galaxies - there, failing to account for extinction in the vicinity of stars would lead to a significant overestimate of the importance of radiation, since one would assume all emergent flux is in the UV/optical).

Within the M1 models, in addition to the physical variations (turning on and off radiation pressure and different wavebands) discussed above, we have also studied the role of the numerical treatment of the IR (as a single bin with grey opacity versus explicitly evolved radiation temperature fields with complicated opacities), which has little effect. In our M1 runs, we have also considered variations in a limited subset of runs of $\mathbf{m 1 0 q}$ of the numerical method used to 'deposit' radiation in the neighbouring cells (weighting by solid angle, as in Hopkins et al. 2018a, or with a simpler kernel weight) and the time-step for star particles (how frequently this is done, as described in Hopkins et al. 2018b, varying between our default stellar-evolution time-step and 10x shorter or $10 x$ longer). None of these variations has a significant effect. We have also varied the 'reduced speed of light' in both $\mathbf{~ m 1 0 q}$ and $\mathbf{m 1 2 i}$ haloes, from $\tilde{c} \sim 300-5000 \mathrm{~km} \mathrm{~s}^{-1}$; the effects are small for $\tilde{c} \gtrsim 500 \mathrm{~km} \mathrm{~s}^{-1}$ (generally smaller, for example, than the differences between M1 and LEBRON methods), consistent with the wellknown result that this should be converged so long as $\tilde{c}$ is faster than other, explicitly resolved speeds in the simulations. However it is not completely negligible: the general sense is that increasing $\tilde{c}$ from $\sim 300-1000 \mathrm{~km} \mathrm{~s}^{-1}$ gives slightly stronger radiative feedback effects (while increasing it beyond this point has little impact), suggesting that at too-small $\tilde{c} \ll 1000 \mathrm{~km} \mathrm{~s}^{-1}$ photons emitted in dense regions (where massive stars form) may spend 'too long' streaming out, making them less efficient on large scales. As shown in Rosdahl et al. (2015) and Hopkins \& Grudic (2018), because the mean-free-path of ionizing/UV photons is not explicitly resolved in the simulations (it is many orders-of-magnitude beyond stateof-the-art resolution), naive implementations of M1 that fail to account for two closely related potential errors in the coupling between photon momentum and gas will underestimate the radiation pressure forces by orders of magnitude. Our default M1 implementation includes the relevant fixes demonstrated in those papers to resolve this issue: but we have considered one test (not shown) of $\mathbf{m 1 0 q}$ removing these fixes (i.e. using the 'M1 (Cellcentred)' implementation described in Hopkins \& Grudic 2018). As expected, the results from the incorrect method are essentially identical to our runs with $\mathrm{M} 1$ removing radiation pressure entirely.

GIZMO also includes two other moments-based methods for RHD, the 'flux-limited diffusion' (FLD; Levermore 1984) and 'optically-thin, variable Eddington tensor' (OTVET; Gnedin \& Abel 2001) methods. These are just the zeroth-moment expansions of the photon transport equation, where one closes the equations at zeroth order by assuming pure diffusion with a 'flux limiter' as opposed to explicitly evolving the flux vector as in the first-moment 'M1' method (the primary difference between FLD and OTVET is whether one assumes an isotropic Eddington tensor in FLD, or the Eddington tensor which would be calculated if all sources were optically thin, in OTVET). Unlike M1, these cannot capture phenomena such as 'shadowing' by optically thick structures; moreover they are actually more computationally expensive owing to a stricter time-step criterion. We therefore did not consider them primarily here; however we have run both $\mathbf{m 1 0 q}$ and $\mathbf{m 1 2 i}$ at intermediate resolution with both methods ( $\mathbf{m 1 2 i}$ only run down to $z \sim 1$ ). The only difference with our 'Default (M1)' runs is the exact form of the photon-propagation step. We find these give very similar results to $\mathrm{M} 1$.

More interesting is the difference between LEBRON and M1, which are fundamentally distinct methods. Recall, neither of these methods is exact in general cases, even at infinite resolution. LEBRON converges to exact solutions in the optically thin regime, independent of the number and distribution of sources, but will only converge to approximate solutions in the optically thick, multiplescattering regime. Conversely, M1 converges to exact solutions in the optically thick multiple-scattering regime, but will incorrectly merge photons and reduce to the diffusion limit in the optically thin regime if there are multiple sources. So we should not regard either of these methods as 'correct.' But since they are exact in essentially opposite limits, it is plausible to suppose they bracket the reasonable range of behaviours. In detail, if we compute the radiative flux at a given frequency, at any given specific point $\mathbf{x}$ in the simulations, it is possible for LEBRON and M1 to diverge by orders-of-magnitude from each other (if, e.g. the point is shadowed by complex, very optically thick structures, where most of the photons are absorbed). However, if we are only concerned with galaxy-scale properties, our comparisons show that the two give broadly similar results. This is because in either method, most of the short-wavelength (UV) photons are absorbed in the ISM near massive stars (while most of the longer wavelength IR photons escape), so the average heating rates and radiation pressure forces, their characteristic spatial and time-scales, are broadly similar. The exact spatial locations where absorption occurs are of secondary importance, and the difference in the dynamics of a region illuminated by a 'modestly attenuated' spectrum (say, $\tau \sim 1-10$ ) versus 'heavily attenuated' $(\tau \sim 10-100$ ) is not important (even though the flux differs by orders of magnitude) because in both cases most 
of the light is blocked and so the resulting radiation effects are weak. ${ }^{17}$

We do tend to find that the effects of radiation are slightly stronger in M1, as compared to LEBRON. In Figs 1 and 2, the 'Default (M1)' dwarfs (m10q, m11b, m11q) have slightly smoother SFHs (with slightly larger SFRs owing to less-bursty/violent SF), while the 'Default (M1)' MW-mass systems (m12i, m12m) have slightly lower late-time SFRs and central circular velocity curve 'spikes' (compared to 'Default (LEBRON)'). Our experiments turning on and off different components of the radiative FB imply this is dominated by the effects of the far-UV/ionizing bands. In fact, the dominant source of the difference appears to lie in how the 'Default (LEBRON)' method calculates absorption of ionizing photons in the immediate vicinity of the emitting star: as described in Paper I, this uses a Stromgren-type approximation. Moving spherically outwards from the star particle, each time a gas element is encountered, the code calculates the number of ionizing photons needed to fully ionize it over the time-step (consuming them), until the 'long range' escape is reached or the photons are exhausted. But imagine a star surrounded by (mostly) low-density gas with one extremely dense (optically thick) 'clump.' In reality, the clump subtends a small area on the sky, so should receive and destroy a small fraction of the ionizing photons; but if it is within the local kernel in LEBRON, it will be encountered in the radial search, and since the number of ionizing photons needed to ionize some volume scales as $\propto n^{2}$, it can essentially 'use up' the full photon budget. Taking an identical snapshot of $\mathbf{~} \mathbf{1 0 q}$ and $\mathbf{m 1 2} \mathbf{i}$, at $z=0.05$, and running it for a very short amount of time with both M1 and LEBRON methods in turn (with no UV background), we have confirmed that in M1 a larger total mass of gas is ionized by the same number of photons emitted from the stars. So ironically, even though LEBRON is formally a non-photon-conserving scheme, it actually tends to artificially reduce the number of viable photons for feedback. This also suggests improvements to the short-range terms in the LEBRON scheme, based on e.g. HEALPIX or other angular tesselation rather than a spherically symmetric assumption, might reduce the discrepancy.

In future work, we will explore simulations using the RHD scheme from Jiang et al. (2014) implemented in GIZMO, which is exact in both optically thin and thick regimes. However it is much more expensive, especially for multiband transport.

Extensive numerical tests of almost every other aspect of these simulations (resolution, force softening, hydrodynamic solvers, etc.) are presented in Hopkins et al. (2018b,a). These all use the 'Default (LEBRON)' method, except where otherwise specified. So extensive resolution tests of this particular method are presented there. As shown in Hopkins et al. (2018b), the simulations do have many predictions which depend on resolution with this default prescription. However, this is not necessarily because the radiation transport for a fixed physical mass configuration is not converged (in fact Hopkins \& Grudic 2018 show quantities like the radiation pressure coupled are reasonably well-converged with these methods

\footnotetext{
${ }^{17}$ Because of the Lagrangian nature of the code here, H II regions are always comparably resolved in gas and stars. Briefly, a Stromgren sphere sourced by $N_{*}$ young star particles of mass $m_{i}$ will fully ionize $\sim$ $2 N_{*}\left(n_{\text {gas }} / 100 \mathrm{~cm}^{-3}\right)^{-1}$ gas resolution elements. Since the maximum densities reached here are comparable to our density threshold for star formation $\left(\sim 1000 \mathrm{~cm}^{-3}\right)$, this is at least marginally resolved for any resolved star cluster. For our highest resolution simulations $\left(m_{i}=250 \mathrm{M}_{\odot}\right)$, H II regions from individual O-stars can be marginally resolved (see discussion in Ma et al. 2015; Su et al. 2018; Wheeler et al. 2019).
}

and this setup). Rather, the gas and stellar distributions exhibit more and more complex substructure at higher resolution, so (naturally) quantities like where and when and how the radiation couples can, in turn, scale as well. Although, as noted above, quantities such as the GMC mass function at the largest masses (which can we resolved), surface densities/optical depths, linewidth-size relation, and related properties do appear to be particularly robust to resolution (see Guszejnov et al. 2017, 2019; Hopkins et al. 2018b likely owing to how these properties are self-regulated by extremely simple bulk galaxy properties like the Toomre mass for a $Q \sim 1$ disc in a turbulent fragmentation cascade (see Hopkins 2012a,b, 2013b; Guszejnov \& Hopkins 2015; Guszejnov et al. 2018). So the most important question, perhaps, is whether and how new (currently un-resolved) scales like protostellar cores will depend on radiative physics beyond the scope explored here.

\subsection{A note on 'sub-grid' models for radiation}

A variety of 'sub-grid' models for stellar feedback do not attempt to explicitly model the salient physical processes, but rather to capture their 'net effects.' These are common (indeed, necessary) in large-volume simulations which cannot resolve the ISM. The most obvious examples are models like Springel \& Hernquist (2003a), Davé, Thompson \& Hopkins (2016), which simply eject mass from a galaxy with some scaling proportional to the star formation rate (and add pressure to dense gas, attributed to un-resolved phase structure). Whether one attributes these scalings to $\mathrm{SNe}$ or radiation or some combination of these and other physics, they are obviously fundamentally distinct from the models here. Essentially, we are trying to predict these effects, using stellar evolution theory (for calculating e.g. SNe rates and energetics, radiative luminosities and spectra) as the 'input.'

We note this because several such models have been used for radiative FB. For example, Agertz et al. (2013) and Agertz \& Kravtsov (2015) add an outward momentum flux in cells immediately adjacent to a star particle scaled to a multiple of $L / c$; Ceverino et al. (2014) add a pressure $P \sim L /\left(c A_{\text {cell }}\right)$ to the hydrodynamic pressure (where $A_{\text {cell }} \sim \Delta x^{2}$ is the cell area) in cells containing star particles $<5$ Myr old; Stinson et al. (2013) add a heating term $\dot{E} \sim L$ to the gas heating/cooling subroutine for the gas containing star particles $<10 \mathrm{Myr}$ old. Although these might represent some consequences of radiative $\mathrm{FB}$, none of these models attempts to actually follow radiation (transport or RHD) explicitly. Our comparisons here indicate that an approximation like that in Agertz \& Kravtsov (2015) might be reasonable for the single-scattering RP (with $\sim 1 / 2 L / c$ or $\sim 1 L_{\mathrm{UV}} / c$ absorbed), in simulations which do not resolve GMCs (since we find most of the single-scattering RP is imparted in the GMCs in which massive stars are born). In fact, Lupi et al. (2018) compare several RHD methods including our LEBRON and M1 algorithms in GIZMO, to such a 'local momentum flux' estimator, and do argue that it is able to capture many of the most important effects (and describe a similar comparison for local photoionization heating). It is unlikely that the approximation in Ceverino et al. (2014) resembles the radiative FB here, since it is only representative of 'radiation pressure' in the infinite optical depth (perfect-trapping), multiple-scattering, greyopacity limit. And the approximation in Stinson et al. (2013) might capture some effects of photoionization heating if $\mathrm{H}$ II regions are un-resolved, but physically in these cases the heating should be restricted to photoheating (i.e. not allowed to heat at $T \gg 10^{4} \mathrm{~K}$ ) from ionizing radiation. 
In any case, it is interesting, but beyond the scope of our study, to explore whether one can define a better sub-grid model for use in lower resolution simulations, or whether capturing the key effects ultimately requires explicitly tracking multiband radiation transport as we do here. For one such study, we refer readers to Lupi et al. (2018).

\section{SUMMARY AND CONCLUSIONS}

We use a survey of $\sim 100$ high-resolution radiation-hydrodynamical cosmological zoom-in simulations of galaxies to study the nature and effects of radiative feedback from stars on galaxy formation. Our simulations span masses from ultrafaint to Milky Way $\left(M_{*} \sim 10^{4}-10^{11} M_{\odot}\right)$, and include the FIRE-2 physical models for ISM microphysics (cooling, chemistry), star formation, and stellar feedback (from supernovae and stellar winds, in addition to radiation). We extensively survey several different radiative feedback 'channels' and wavelength ranges, and consider two fundamentally distinct numerical radiation-hydrodynamics methods, in order to identify the most important and robust results. We note that this is a companion paper to Paper I and Paper II, where more general numerical (e.g. resolution, hydro solvers) and mechanical feedback (SNe \& stellar mass-loss) methods are explored in detail.

\subsection{Overview: different radiative feedback mechanisms in galaxy formation}

(i) Averaged over the entire life of a galaxy, most of the emitted far-UV/ionizing radiation $(\sim 1 / 2$ the total bolometric) is absorbed. Relatively little optical/NIR/FIR is absorbed. Total absorption increases with galaxy mass (as dust masses and densities increase), but the effect is weak because of efficient neutral gas absorption of ionizing photons in even metal-free galaxies.

(ii) As a result, the most important feedback mechanisms, in a galaxy-lifetime-averaged sense, are photoionization heating, and single-scattering radiation pressure from UV and ionizing photons. Although we did not study it here, it is possible that resonant Ly $\alpha$ scattering in metal-poor dwarfs could produce similar effects.

(iii) Photoelectric and IR thermal dust/collisional heating, while important for phase structure in dense, cold $\left(T \ll 10^{4} \mathrm{~K}\right)$ gas, have weak effects on galactic scales (thermal pressure is always weak in the cold gas, compared to e.g. turbulence). Likewise, since we ignore Pop III (metal-free) star formation, Lyman-Werner radiation also plays a minor role (as molecular cooling has essentially no effect on star formation in the presence of even trace metals; see Glover \& Clark 2012). We showed in Paper I that even much more radical changes to cooling physics in $T<10^{4} \mathrm{~K}$ gas have negligible effects. Compton heating from soft/hard X-rays emitted by LMXBs/HMXBs also plays a minor role: the flux is too low to compete with cooling rates in hot gas.

(iv) A more detailed 'breakdown' of ionizing radiation into a multiband treatment, e.g. separately following HeI, HeII, and HI, makes relatively small differences compared to following ionizing photons in a single-band approximation, with a mean SED calculated for young stellar populations. This does not mean He II ionization has no effects, but simply that they can be captured (to leading order) by a mean local+UVB SED treatment.

(v) Multiple-scattering of IR photons produces weak effects, in a galaxy-lifetime-averaged sense, at achievable cosmological resolutions. In metal-poor dwarfs, IR optical depths are almost always small. In MW-mass systems, most photons are absorbed in 'typical' GMCs which are optically thin in the IR $\left(\tau_{\mathrm{IR}} \sim 0.1\right)$ on average. Only a small fraction of the light, in e.g. galaxy nuclei in starbursts or dense, high-redshift clouds, is emitted in regions with $\tau_{\mathrm{IR}} \gg 1$ where multiple-scattering is potentially important. However this could change if we resolved extremely small, dense structures in cores, although this is likely not where most radiation is emitted.

\subsection{Important dynamical effects of radiation on galaxies}

We summarize the dynamical effects of the key radiative feedback channels identified above. In our study, we focused on bulk galaxy properties including: SFRs; stellar masses; metallicities; stellar, baryonic and dark matter density profiles; rotation curves; and morphology. We also briefly discussed, but did not study in detail, quantities such as outflow rates and ISM phase structure.

\subsubsection{In dwarf galaxies}

(i) The most important radiative feedback effect in dwarfs is photoionization heating by the metagalactic background (UVB). Even in galaxies with $V_{\max } \sim 50 \mathrm{~km} \mathrm{~s}^{-1}$, much more massive than the threshold where the UVB 'quenches' $\left(\sim 10-20 \mathrm{~km} \mathrm{~s}^{-1}\right)$, removing the UVB leads to order-of-magnitude enhanced SFRs (much larger than observed) owing to additional late-time cooling and accretion that is otherwise suppressed.

(ii) Radiation pressure and local photoheating play a similar role to one another: both 'smooth out' star formation by providing a 'gentle' form of feedback that can support warm or cool gas which would otherwise lose its pressure support and collapse under selfgravity. If we remove these, the star formation becomes substantially more 'violent' and 'bursty' because cool/warm gas more rapidly fragments into GMCs, whose collapse is only halted by SNe after they turn much of their mass into stars (similar conclusions have been reached using idealized, non-cosmological simulations with entirely different numerical methods and treatments of mechanical feedback; Kannan et al. 2014b; Rosdahl et al. 2015; Emerick et al. 2018; Kimm et al. 2018). Within the galaxy, GMC and 'star forming clump' lifetimes and star formation efficiencies are obviously strongly modified - the 'no radiative feedback' prediction is already ruled out by resolved GMC observations (compare Lee, Miville-Deschênes \& Murray 2016; Grudić et al. 2018c). But also, if we remove radiative feedback, the more violent $\mathrm{SNe}$ feedback makes the galaxies more metal poor (metallicities are suppressed by $\sim 0.5 \mathrm{dex}$, in conflict with the observed mass-metallicity relation; Ma et al. 2016a) and more baryon-poor (lower stellar mass by a factor $\sim 1.5-2$, and substantially more gas-poor) with almost no residual cool gas. These effects are notably exacerbated (with excessive early-time star formation followed by 'self-quenching' from explosive $\mathrm{SNe}$ feedback) if we also remove 'early' stellar mass-loss (e.g. fast $\mathrm{O} / \mathrm{B}$ winds), which carry a momentum flux similar to radiation.

\subsubsection{In massive (Milky Way-mass) galaxies}

(i) Photoheating by the UVB plays a negligible role in the evolution of the primary galaxy (though it is important for the dwarf satellites as described above), once it is massive $\left(V_{\max } \gtrsim\right.$ $100 \mathrm{~km} \mathrm{~s}^{-1}$ ).

(ii) Radiation pressure and local photoheating again play a similar role to each other: by pre-processing GMCs and dense starforming gas, they allow SNe to more easily able to escape, overlap 
and generate superbubbles, especially in the denser central regions of galaxies. Removing them leads to substantially more compact, dense bulges, and steep central rotation curves, in conflict with observations. Radiative FB is important within galaxies on short time-scales regulating the rate of conversion of dense gas into stars (e.g. the position of the galaxy on the Schmidt-Kennicutt relation), as previous studies have shown (Wise \& Abel 2008; Hopkins et al. 2011; Krumholz, Klein \& McKee 2011; Federrath 2015; Trujillo-Gomez et al. 2015). More dramatically, without this or some other strong 'early feedback' acting before SNe explode, massive GMCs turn almost all their mass into stars leaving hyperdense bound relics, predicting galaxies dominated entirely by dense star clusters (consistent with many previous GMC-scale studies, e.g. Fall, Krumholz \& Matzner 2010; Colín et al. 2013; Howard et al. 2016; Rosen et al. 2016; Kim et al. 2017; Grudić et al. 2018b).

(iii) Of course, without local photoheating, one also cannot correctly predict the distribution of ISM phases (the warm medium in particular), which is maintained by these processes.

\subsection{Numerical methods and caveats}

RHD remains a numerical frontier: almost all RHD methods which are efficient enough to use in high-resolution cosmological simulations are approximate in some manner, and uncertainty remains regarding the role this plays in radiative feedback. We cannot definitively resolve this since the methods here are among those approximate classes; however we have compared fundamentally distinct methods and choices within those methods.

(i) Our results are qualitatively similar using either the LEBRON or M1 numerical RHD methods. LEBRON is a ray-based algorithm which is exact in the optically thin limit (independent of source number), but fails to capture shadowing and exact photonconservation in the optically thick, multiple-scattering limits. M1 is a moments-based algorithm which is photon-conserving and exact in the optically thick, multiple-scattering limit but cannot capture the optically thin limit with multiple sources (intersecting rays 'collide' and diffuse out). Both are approximate, but valid in essentially opposite limits. To first order, we find they give similar results. In detail, M1 shows slightly stronger radiative FB effects: this appears to owe (primarily) to the fact that LEBRON artificially allows dense 'clumps' near massive stars to consume too many ionizing/UV photons. We have also considered limited comparisons of other, less-accurate moments-based methods (e.g. FLD) which are similar to M1.

(ii) Reassuringly, our results are also consistent with a growing number of multifrequency RHD studies using a range of different numerical RHD and hydro methods to treat the same radiative FB mechanisms (see e.g. Kannan et al. 2014b; Rosdahl et al. 2015; Emerick et al. 2018; Kimm et al. 2018).

(iii) We stress that the M1 implementation here involves the 'face-centred' formulation which resolves the numerical errors identified in Rosdahl et al. (2015) and Hopkins \& Grudic (2018). Without these, the radiation pressure is strongly artificially suppressed.

(iv) While Paper I (and this paper to a lesser extent) show our conclusions are robust over factors of $\sim 100$ in mass resolution, we stress that this does not mean they are formally 'converged.' At much higher resolution, new physics, including resolving smaller scale substructure in gas (e.g. small 'holes' in compact H II regions) and the spatial distribution of massive stars within star clusters may have important effects on how radiative FB acts upon natal GMCs. (v) We show that some 'early feedback' (feedback from massive stars before $\mathrm{SNe}$ explode) is critical for regulating collapse of GMCs, bursty/violent SF, and galaxy morphologies. However, for the default simulations here this support can be provided by a mix of $\mathrm{O} / \mathrm{B}$ stellar mass-loss, single-scattering radiation pressure, and warm photoionized gas in $\mathrm{H}$ II regions. From a numerical point of view, these are somewhat degenerate if we only consider galaxyscale properties (all act on similar small time and spatial scales with similar momentum fluxes). Their relative importance, if the coupling is occurring near the resolution limit, can be sensitive to numerical choices (e.g. whether one accounts for un-resolved multiple-scattering or leakage, or for 'trapping' and pressure-driven work done by stellar wind bubbles) - for this reason we find somewhat different results in FIRE-2 versus FIRE-1. Simulations and observations of smaller (GMC and star cluster) scales are clearly needed to robustly address the relative roles played here.

\subsection{Additional caveats, missing physics, and future work}

In addition to the numerical caveats above, we stress that our simulation set is necessarily limited.

(i) As we noted above, it is likely that some physics here (e.g. infrared multiple-scattering or Lyman-Werner radiation) could be very important in special environments and/or times in the life of galaxies (e.g. nuclear starbursts or circum-AGN environments, or in the first metal-free stars), even if it does not alter global properties of the 'typical' galaxy at $z=0$ (see e.g. Thompson, Quataert \& Murray 2005; Costa et al. 2018b).

(ii) Our treatment of the metagalactic UVB is not selfconsistent because actually predicting the UVB requires volumes $\gg(100 \mathrm{Mpc})^{3}$, impossible to achieve at our resolution: one possible approach is to use large-scale (low-resolution) studies to model e.g. fluctuations in the local UVB field in e.g. QSO proximity zones (or patches surrounding different structures during the process of reionization, where the UVB may be highly inhomogeneous), then use those models for zoom-in simulations to self-consistently predict e.g. UV escape fractions.

(iii) We have also neglected some potentially important radiative feedback channels from Section 2, e.g. overionization of metalspecies in the CGM, or multiple-scattering in resonance lines. The former requires non-equilibrium chemistry for the metals, which will be studied in future work (Richings et al., in preparation), but preliminary studies suggest it may only be significant in the near vicinity of AGN or extreme starbursts (Richings \& Schaye 2016; Oppenheimer et al. 2018). The latter is generally not believed to be important on galaxy scales (though it is probably critical for wind-launching in stellar photospheres and AGN accretion discs), except perhaps from resonant Ly $\alpha$ scattering; but following this requires Ly $\alpha$ radiative transfer which cannot be handled by any of the default RHD methods here (it will be studied in future work, Ma et al., in preparation; but see e.g. Dijkstra \& Loeb 2009; Smith et al. 2017; Kimm et al. 2018).

(iv) We neglect a potentially critically important radiative FB channel, in AGN. AGN are generally sub-dominant in luminosity in low-mass (sub-MW-mass) galaxies, at almost all times and redshifts, relative to stars - so this is probably a reasonable approximation for the galaxies we study here. But for more massive galaxies, the radiation pressure, photoheating, and Compton heating from AGN can easily dominate that from stars by orders of magnitude. It is likely that in these regimes, bright AGN or quasars have very important radiative feedback effects not captured here 
(see e.g. Proga 2000; Murray et al. 2005; Sazonov et al. 2005; Kurosawa \& Proga 2008; Choi et al. 2014; Hopkins et al. 2016; Brennan et al. 2018; Costa et al. 2018a).

(v) We assume perfect momentum re-distribution from dust to gas, when photons are absorbed by dust (i.e. assume dust and gas move together), in addition to a constant dust-to-metals ratio. While unimportant for ionizing photons if neutral gas dominates the opacity, in more massive galaxies at longer wavelengths the dust opacities dominate. But Squire \& Hopkins (2018b,a), Hopkins \& Squire $(2018 \mathrm{a}, \mathrm{b})$ recently showed that this scenario (accelerating one of either gas or dust, and relying on drag or Lorentz forces to 'pull' the other along) is violently unstable, with the ensuing instabilities driving strong turbulence and segregation of the dust and gas. Whether it is in fact possible to have 'dust-driven' outflows requires further investigation in light of these previously unrecognized instabilities.

We also stress, once more, that our conclusions here apply to global, galaxy-scale properties. The radiative physics controlling e.g. proto-stellar evolution and the initial mass function, or star cluster formation, or AGN accretion, will be distinct, as the characteristic spatial scales, time-scales relative to stellar evolution, opacities, densities, and wavelengths where most of the light is emitted differ enormously.

Likewise, it should be obvious that the detailed chemical state of a galaxy, and observational diagnostics of this state and the stellar emission itself, depend directly on the radiation from stars (e.g. the radiative environment determines quantities like the $\mathrm{H} \alpha$, O III, $\mathrm{CO}$, C II luminosities and excitation, the UV/optical/IR continuum, etc.). Our goal here was not to explore these observables as 'tracers,' but rather to ask whether and how stellar radiation alters the formation, evolution, and dynamics of bulk galaxy properties.

\section{ACKNOWLEDGEMENTS}

We thank Eliot Quataert, Alexander Richings, and Alexander Gurvich, with whom we have had a number of useful discussions on topics here. Support for PFH and co-authors was provided by an Alfred P. Sloan Research Fellowship, NSF Collaborative Research Grant \#1715847 and CAREER grant \#1455342, and NASA grants NNX15AT06G, JPL 1589742, 17-ATP17-0214. AW was supported by NASA, through ATP grant 80NSSC18K1097, and HST grants GO-14734 and AR-15057 from STScI. DK was supported by NSF grant AST-1715101 and the Cottrell Scholar Award from the Research Corporation for Science Advancement. Numerical calculations were run on the Caltech compute cluster 'Wheeler,' allocations from XSEDE TG-AST130039 and PRAC NSF.1713353 supported by the NSF, and NASA HEC SMD-16-7592.

\section{REFERENCES}

Agertz O., Kravtsov A. V., 2015, ApJ, 804, 18

Agertz O., Kravtsov A. V., Leitner S. N., Gnedin N. Y., 2013, ApJ, 770, 25

Barkana R., Loeb A., 2001, Phys. Rep., 349, 125

Bate M. R., 2012, MNRAS, 419, 3115

Bate M. R., Tricco T. S., Price D. J., 2014, MNRAS, 437, 77

Behroozi P. S., Conroy C., Wechsler R. H., 2010, ApJ, 717, 379

Bieri R., Dubois Y., Rosdahl J., Wagner A., Silk J., Mamon G. A., 2017, MNRAS, 464, 1854

Blitz L., 1993, in Levy E. H., Lunine J. I., eds, Protostars and Planets III. University of Arizona Press, Tucson, p. 125

Bolatto A. D., Leroy A. K., Rosolowsky E., Walter F., Blitz L., 2008, ApJ, 686, 948
Bournaud F., Elmegreen B. G., Teyssier R., Block D. L., Puerari I., 2010, MNRAS, 409, 1088

Brennan R., Choi E., Somerville R. S., Hirschmann M., Naab T., Ostriker J. P., 2018, ApJ, 860, 14

Bryan G. L., Norman M. L., 1998, ApJ, 495, 80

Cantalupo S., 2010, MNRAS, 403, L16

Ceverino D., Klypin A., Klimek E. S., Trujillo-Gomez S., Churchill C. W., Primack J., Dekel A., 2014, MNRAS, 442, 1545

Chan T. K., Kereš D., Oñorbe J., Hopkins P. F., Muratov A. L., FaucherGiguère C.-A., Quataert E., 2015, MNRAS, 454, 2981

Chan T. K., Kereš D., Wetzel A., Hopkins P. F., Faucher-Giguère C.-A., El-Badry K., Garrison-Kimmel S., Boylan-Kolchin M., 2018, MNRAS, 478, 906

Choi E., Naab T., Ostriker J. P., Johansson P. H., Moster B. P., 2014, MNRAS, 442,440

Cole S., Lacey C. G., Baugh C. M., Frenk C. S., 2000, MNRAS, 319, 168

Colín P., Vázquez-Semadeni E., Gómez G. C., 2013, MNRAS, 435, 1701

Conroy C., Wechsler R. H., Kravtsov A. V., 2006, ApJ, 647, 201

Corbett Moran C., Grudić M. Y., Hopkins P. F., 2018, MNRAS , preprint (arXiv:1803.06430)

Costa T., Rosdahl J., Sijacki D., Haehnelt M. G., 2018a, MNRAS, 473, 4197

Costa T., Rosdahl J., Sijacki D., Haehnelt M. G., 2018b, MNRAS, 479, 2079

Davé R., Thompson R., Hopkins P. F., 2016, MNRAS, 462, 3265

Davis S. W., Jiang Y.-F., Stone J. M., Murray N., 2014, ApJ, 796, 107

Dijkstra M., Loeb A., 2009, MNRAS, 396, 377

Dobbs C. L., Glover S. C. O., Clark P. C., Klessen R. S., 2008, MNRAS, 389, 1097

Dobbs C. L., Burkert A., Pringle J. E., 2011, MNRAS, 413, 2935

Dopcke G., Glover S. C. O., Clark P. C., Klessen R. S., 2013, ApJ, 766, 103

Draine B. T., 2011, Physics of the Interstellar and Intergalactic Medium. Princeton Univ. Press, Princeton, NJ, USA

Emerick A., Bryan G. L., Mac Low M.-M., Côté B., Johnston K. V., O’Shea B. W., 2018, ApJ, 869, 94

Evans N. J. II, 1999, ARA\&A, 37, 311

Evans N. J. et al., 2009, ApJS, 181, 321

Fall S. M., Krumholz M. R., Matzner C. D., 2010, ApJ, 710, L142

Faucher-Giguère C.-A., Lidz A., Zaldarriaga M., Hernquist L., 2009, ApJ, 703,1416

Faucher-Giguère C.-A., Kereš D., Dijkstra M., Hernquist L., Zaldarriaga M., 2010, ApJ, 725, 633

Faucher-Giguère C.-A., Kereš D., Ma C.-P., 2011, MNRAS, 417, 2982

Federrath C., 2015, MNRAS, 450, 4035

Federrath C., Klessen R. S., 2012, ApJ, 761, 156

Fielding D., Quataert E., Martizzi D., 2018, MNRAS, 481, 3325

Gentry E. S., Krumholz M. R., Dekel A., Madau P., 2017, MNRAS, 465, 2471

Glover S. C. O., Clark P. C., 2012, MNRAS, 421, 9

Gnedin N. Y., Abel T., 2001, New Astron., 6, 437

Grassi T., Bovino S., Schleicher D. R. G., Prieto J., Seifried D., Simoncini E., Gianturco F. A., 2014, MNRAS, 439, 2386

Grudić M. Y., Guszejnov D., Hopkins P. F., Lamberts A., Boylan-Kolchin M., Murray N., Schmitz D., 2018a, MNRAS, 481, 688

Grudić M. Y., Hopkins P. F., Faucher-Giguère C.-A., Quataert E., Murray N., Kereš D., 2018b, MNRAS, 475, 3511

Grudić M. Y., Hopkins P. F., Lee E. J., Murray N., Faucher-Giguère C.-A., Johnson L. C., 2019, MNRAS, 488, 1501

Grudić M. Y., Hopkins P. F., Quataert E., Murray N., 2019, MNRAS, 483 , 5548

Guszejnov D., Hopkins P. F., 2015, MNRAS, 450, 4137

Guszejnov D., Krumholz M. R., Hopkins P. F., 2016, MNRAS, 458, 673

Guszejnov D., Hopkins P. F., Krumholz M. R., 2017, MNRAS, 468, 4093

Guszejnov D., Hopkins P. F., Ma X., 2017, MNRAS, 472, 2107

Guszejnov D., Hopkins P. F., Grudić M. Y., 2018, MNRAS, 477, 5139

Guszejnov D., Grudić M. Y., Offner S. S. R., Boylan-Kolchin M., FaucherGigère C.-A., Wetzel A., Benincasa S. M., Loebman S., 2019, preprint (arXiv:1910.01163)

Haid S., Walch S., Naab T., Seifried D., Mackey J., Gatto A., 2016, MNRAS, 460,2962 
Hansen C. E., Klein R. I., McKee C. F., Fisher R. T., 2012, ApJ, 747, 22 Harper-Clark E., Murray N., 2011, in Alves J., Elmegreen B. G., Girart J. M., Trimble V., eds, Computational Star Formation, Vol. 270. Cambridge Univ., Press, Cambridge, UK, p. 235

Harris W. E., 1996, AJ, 112, 1487

Heckman T. M., Lehnert M. D., Strickland D. K., Armus L., 2000, ApJS, 129,493

Hopkins P. F., 2012a, MNRAS, 423, 2016

Hopkins P. F., 2012b, MNRAS, 423, 2037

Hopkins P. F., 2013a, MNRAS, 428, 2840

Hopkins P. F., 2013b, MNRAS, 430, 1653

Hopkins P. F., 2015, MNRAS, 450, 53

Hopkins P. F., 2016, MNRAS, 462, 576

Hopkins P. F., 2017, MNRAS, 466, 3387

Hopkins P. F. et al., 2018, MNRAS, 480, 800

Hopkins P. F., Raives M. J., 2016, MNRAS, 455, 51

Hopkins P. F., Squire J., 2018a, MNRAS, 480, 2813

Hopkins P. F., Squire J., 2018b, MNRAS, 479, 4681

Hopkins P. F., Quataert E., Murray N., 2011, MNRAS, 417, 950

Hopkins P. F., Quataert E., Murray N., 2012a, MNRAS, 421, 3522

Hopkins P. F., Quataert E., Murray N., 2012b, MNRAS, 421, 3488

Hopkins P. F., Narayanan D., Murray N., Quataert E., 2013, MNRAS, 433, 69

Hopkins P. F., Narayanan D., Murray N., 2013, MNRAS, 432, 2647

Hopkins P. F., Kereš D., Oñorbe J., Faucher-Giguère C.-A., Quataert E., Murray N., Bullock J. S., 2014, MNRAS, 445, 581

Hopkins P. F., Torrey P., Faucher-Giguère C.-A., Quataert E., Murray N., 2016, MNRAS, 458, 816

Hopkins P. F. et al., 2018a, MNRAS, 477, 1578

Hopkins P. F. et al., 2018b, MNRAS, 480, 800

Hopkins P. F. et al., 2019, MNRAS, preprint (arXiv:1905.04321)

Howard C. S., Pudritz R. E., Harris W. E., 2016, MNRAS, 461, 2953

Hu C.-Y., Naab T., Walch S., Glover S. C. O., Clark P. C., 2016, MNRAS, 458,3528

Hu C.-Y., Naab T., Glover S. C. O., Walch S., Clark P. C., 2017, MNRAS, 471,2151

Jiang Y.-F., Davis S. W., Stone J. M., 2013, ApJ, 763, 102

Jiang Y.-F., Stone J. M., Davis S. W., 2014, ApJS, 213, 7

Ji A. P., Frebel A., Bromm V., 2014, ApJ, 782, 95

Kannan R., Stinson G. S., Macciò A. V., Brook C., Weinmann S. M., Wadsley J., Couchman H. M. P., 2014a, MNRAS, 437, 3529

Kannan R. et al., 2014b, MNRAS, 437, 2882

Kannan R., Vogelsberger M., Marinacci F., McKinnon R., Pakmor R., Springel V., 2019, MNRAS, 485, 117

Katz N., Weinberg D. H., Hernquist L., 1996, ApJS, 105, 19

Kennicutt R. C. Jr, 1998, ApJ, 498, 541

Kereš D., Katz N., Davé R., Fardal M., Weinberg D. H., 2009, MNRAS, 396, 2332

Kim J.-. hoon . et al., 2018, MNRAS, 474, 4232

Kim J.-G., Kim W.-T., Ostriker E. C., Skinner M. A., 2017, ApJ, 851, 93

Kimm T., Haehnelt M., Blaizot J., Katz H., Michel-Dansac L., Garel T., Rosdahl J., Teyssier R., 2018, MNRAS, 475, 4617

Kroupa P., 2002, Science, 295, 82

Krumholz M. R., Gnedin N. Y., 2011, ApJ, 729, 36

Krumholz M. R., Thompson T. A., 2012, ApJ, 760, 155

Krumholz M. R., Klein R. I., McKee C. F., 2011, ApJ, 740, 74

Kuiper R., Klahr H., Beuther H., Henning T., 2012, A\&A, 537, A122

Kurosawa R., Proga D., 2008, ApJ, 674, 97

Lamberts A., Garrison-Kimmel S., Clausen D. R., Hopkins P. F., 2016, MNRAS, 463, L31

Lamberts A. et al., 2018, MNRAS, 480, 2704

Lee E. J., Miville-Deschênes M.-A., Murray N. W., 2016, ApJ, 833, 229

Leitherer C. et al., 1999, ApJS, 123, 3

Levermore C. D., 1984, J. Quant. Spectrosc. Radiat. Transfer, 31, 149

Lopez L. A., Krumholz M. R., Bolatto A. D., Prochaska J. X., Ramirez-Ruiz E., 2011, ApJ, 731, 91

Lupi A., Bovino S., Capelo P. R., Volonteri M., Silk J., 2018, MNRAS, 474, 2884
Ma X., Kasen D., Hopkins P. F., Faucher-Giguère C.-A., Quataert E., Kerě̌ D., Murray N., 2015, MNRAS, 453, 960

Ma X., Hopkins P. F., Faucher-Giguère C.-A., Zolman N., Muratov A. L., Kereš D., Quataert E., 2016a, MNRAS, 456, 2140

Ma X., Hopkins P. F., Kasen D., Quataert E., Faucher-Giguère C.-A., Kereš D., Murray N., Strom A., 2016b, MNRAS, 459, 3614

Ma X. et al., 2018, MNRAS, 478, 1694

Martin C. L., 1999, ApJ, 513, 156

Martin C. L., Scannapieco E., Ellison S. L., Hennawi J. F., Djorgovski S. G., Fournier A. P., 2010, ApJ, 721, 174

Morrison R., McCammon D., 1983, ApJ, 270, 119

Moster B. P., Somerville R. S., Maulbetsch C., van den Bosch F. C., Macciò A. V., Naab T., Oser L., 2010, ApJ, 710, 903

Muratov A. L., Kereš D., Faucher-Giguère C.-A., Hopkins P. F., Quataert E., Murray N., 2015, MNRAS, 454, 2691

Murray N., 2011, ApJ, 729, 133

Murray N., Quataert E., Thompson T. A., 2005, ApJ, 618, 569

Murray N., Quataert E., Thompson T. A., 2010, ApJ, 709, 191

Offner S. S. R., Klein R. I., McKee C. F., Krumholz M. R., 2009, ApJ, 703, 131

Offner S. S. R., Clark P. C., Hennebelle P., Bastian N., Bate M. R., Hopkins P. F., Moraux E., Whitworth A. P., 2014, in Beuther H., Klessen R. S., Dullemond C. P., Henning Th., eds, Protostars and Planets VI. University of Arizona Press, Tucson

Oklopčić A., Hopkins P. F., Feldmann R., Kereš D., Faucher-Giguère C.-A., Murray N., 2017, MNRAS, 465, 952

Oñorbe J., Boylan-Kolchin M., Bullock J. S., Hopkins P. F., Kereš D., Faucher-Giguère C.-A., Quataert E., Murray N., 2015, MNRAS, 454, 2092

Oppenheimer B. D., Segers M., Schaye J., Richings A. J., Crain R. A., 2018, MNRAS, 474, 4740

Orr M. E. et al., 2018, MNRAS, 478, 3653

Peng E. W. et al., 2008, ApJ, 681, 197

Pettini M., Madau P., Bolte M., Prochaska J. X., Ellison S. L., Fan X., 2003, ApJ, 594, 695

Power C., Navarro J. F., Jenkins A., Frenk C. S., White S. D. M., Springel V., Stadel J., Quinn T., 2003, MNRAS, 338, 14

Proga D., 2000, ApJ, 538, 684

Raskutti S., Ostriker E. C., Skinner M. A., 2016, ApJ, 829, 130

Rice T. S., Goodman A. A., Bergin E. A., Beaumont C., Dame T. M., 2016, ApJ, 822, 52

Richings A. J., Schaye J., 2016, MNRAS, 458, 270

Rosdahl J., Teyssier R., 2015, MNRAS, 449, 4380

Rosdahl J., Blaizot J., Aubert D., Stranex T., Teyssier R., 2013, MNRAS, 436, 2188

Rosdahl J., Schaye J., Teyssier R., Agertz O., 2015, MNRAS, 451, 34

Rosen A. L., Krumholz M. R., McKee C. F., Klein R. I., 2016, MNRAS, 463, 2553

Roth N., Kasen D., Hopkins P. F., Quataert E., 2012, ApJ, 759, 36

Roškar R., Teyssier R., Agertz O., Wetzstein M., Moore B., 2014, MNRAS, 444,2837

Sales L. V., Marinacci F., Springel V., Petkova M., 2014, MNRAS, 439, 2990

Sato T., Martin C. L., Noeske K. G., Koo D. C., Lotz J. M., 2009, ApJ, 696, 214

Sazonov S. Y., Ostriker J. P., Sunyaev R. A., 2004, MNRAS, 347, 144

Sazonov S. Y., Ostriker J. P., Ciotti L., Sunyaev R. A., 2005, MNRAS, 358, 168

Semenov D., Henning T., Helling C., Ilgner M., Sedlmayr E., 2003, A\&A, 410,611

Semenov V. A., Kravtsov A. V., Gnedin N. Y., 2018, ApJ, 861, 4

Shetty R., Ostriker E. C., 2008, ApJ, 684, 978

Skinner M. A., Ostriker E. C., 2015, ApJ, 809, 187

Smith A., Bromm V., Loeb A., 2017, MNRAS, 464, 2963

Smith A., Tsang B. T.-H., Bromm V., Milosavljević M., 2018, MNRAS, 479, 2065

Smith A., Ma X., Bromm V., Finkelstein S. L., Hopkins P. F., FaucherGiguère C.-A., Kereš D., 2019, MNRAS, 484, 39 
Solomon P. M., Rivolo A. R., Barrett J., Yahil A., 1987, ApJ, 319, 730

Somerville R. S., Primack J. R., 1999, MNRAS, 310, 1087

Songaila A., 2005, AJ, 130, 1996

Springel V., Hernquist L., 2003a, MNRAS, 339, 289

Springel V., Hernquist L., 2003b, MNRAS, 339, 312

Squire J., Hopkins P. F., 2018a, MNRAS, 477, 5011

Squire J., Hopkins P. F., 2018b, ApJ, 856, L15

Steidel C. C., Erb D. K., Shapley A. E., Pettini M., Reddy N., Bogosavljević M., Rudie G. C., Rakic O., 2010, ApJ, 717, 289

Stinson G. S., Brook C., Macciò A. V., Wadsley J., Quinn T. R., Couchman H. M. P., 2013, MNRAS, 428, 129

Su K.-Y., Hopkins P. F., Hayward C. C., Faucher-Giguère C.-A., Kereš D., Ma X., Robles V. H., 2017, MNRAS, 471, 144

Su K.-Y. et al., 2018, MNRAS, 480, 1666

Takeuchi S., Ohsuga K., Mineshige S., 2014, PASJ, 66, 48

Tasker E. J., 2011, ApJ, 730, 11

Tasker E. J., Bryan G. L., 2008, ApJ, 673, 810

Thompson T. A., Quataert E., Murray N., 2005, ApJ, 630, 167

Thoul A. A., Weinberg D. H., 1996, ApJ, 465, 608

Tielens A. G. G. M., 2005, The Physics and Chemistry of the Interstellar Medium. Cambridge Univ. Press, Cambridge, UK

Torrey P., Hopkins P. F., Faucher-Giguère C.-A., Vogelsberger M., Quataert E., Kereš D., Murray N., 2017, MNRAS, 467, 2301

Trujillo-Gomez S., Klypin A., Colin P., Ceverino D., Arraki K., Primack J., 2015, MNRAS, 446, 1140

Tsang B. T.-H., Milosavljević M., 2015, MNRAS, 453, 1108

Walch S. et al., 2015, MNRAS, 454, 238

Wheeler C. et al., 2017, MNRAS, 465, 2420

Wheeler C. et al., 2019, MNRAS, 490, 4447

Williams J. P., McKee C. F., 1997, ApJ, 476, 166

Wise J. H., Abel T., 2008, ApJ, 685, 40

Wise J. H., Abel T., Turk M. J., Norman M. L., Smith B. D., 2012, MNRAS, 427, 311

Wolfire M. G., Hollenbach D., McKee C. F., Tielens A. G. G. M., Bakes E. L. O., 1995, ApJ, 443, 152

Zhang D., Davis S. W., 2017, ApJ, 839, 54

Zhang D., Davis S. W., Jiang Y.-F., Stone J. M., 2018, ApJ, 854, 110

Zuckerman B., Evans N. J. II, 1974, ApJ, 192, L149

\section{APPENDIX: SOURCE LUMINOSITIES AND OPACITIES}

\section{A1 'Default FIRE'}

Our 'default' RT network uses the five-band transport described in detail in Paper I. For completeness we provide the adopted source luminosities and opacities here, determined as described in Paper I by integrating over the relevant bands after computing detailed spectra from standard stellar evolution models (the same models used to compute all feedback quantities). For sources, define the light-to-mass ratio in a given band $\Psi_{v}$, in units of $L_{\odot} / \mathrm{M}_{\odot}$. Then the bolometric $\Psi_{\text {bol }}=1136.59$ for $t_{\mathrm{Myr}}<$ 3.5 , and $\Psi_{\text {bol }}=1500 \exp \left[-4.145 x+0.691 x^{2}-0.0576 x^{3}\right]$ with $x \equiv \log _{10}\left(t_{\mathrm{Myr}} / 3.5\right)$ for $t_{\mathrm{Myr}}>3.5$. In mid/far-IR, $\Psi_{\mathrm{IR}}=0$. In optical/NIR, $\Psi_{\mathrm{opt}}=f_{\mathrm{opt}} \Psi_{\mathrm{bol}}$ with $f_{\mathrm{opt}}=0.09$ for $t_{\mathrm{Myr}}<2.5 ; f_{\mathrm{Opt}}=$ $0.09\left(1+\left[\left(t_{\mathrm{Myr}}-2.5\right) / 4\right]^{2}\right)$ for $2.5<t_{\mathrm{Myr}}<6 ; f_{\mathrm{Opt}}=1-0.841 /(1+$ $\left.\left[\left(t_{\mathrm{Myr}}-6\right) / 300\right]\right)$ for $t_{\mathrm{Myr}}>6$. In FUV (photoelectric band) $\Psi_{\mathrm{FUV}}=$ $271\left[1+\left(t_{\mathrm{Myr}} / 3.4\right)^{2}\right]$ for $t_{\mathrm{Myr}}<3.4 ; \Psi_{\mathrm{FUV}}=572\left(t_{\mathrm{Myr}} / 3.4\right)^{-1.5}$ for $t_{\mathrm{Myr}}>3.4$. In the ionizing band $\Psi_{\text {ion }}=500$ for $t_{\mathrm{Myr}}<3.5$; $\Psi_{\text {ion }}=60\left(t_{\mathrm{Myr}} / 3.5\right)^{-3.6}+470\left(t_{\mathrm{Myr}} / 3.5\right)^{0.045-1.82 \ln t_{\mathrm{Myr}}}$ for $3.5<$ $t_{\mathrm{Myr}}<25 ; \Psi_{\text {ion }}=0$ for $t_{\mathrm{Myr}}>25$. In NUV, $\Psi_{\mathrm{NUV}}=\Psi_{\text {bol }}-\left(\Psi_{\mathrm{IR}}\right.$ $\left.+\Psi_{\mathrm{opt}}+\Psi_{\mathrm{FUV}}+\Psi_{\text {ion }}\right)$. The adopted flux-mean dust opacities are $\left(\kappa_{\mathrm{FUV}}, \kappa_{\mathrm{NUV}}, \kappa_{\mathrm{opt}}, \kappa_{\mathrm{IR}}\right)=(2000,1800,180,10)\left(Z / Z_{\odot}\right) \mathrm{cm}^{2} \mathrm{~g}^{-1}$.
As described in Paper I, Appendices A and B, the photoelectric and photoionization terms are coupled directly to the gas chemistry and radiative heating/cooling subroutines.

\section{A2 Extended network}

As described in the text, we also (briefly) consider a more extended network. This includes the same optical/NIR $\left(\Psi_{\text {opt }}\right)$ and NUV ( $\left.\Psi_{\mathrm{NUV}}\right)$ bands as above, but a more complex treatment of the photoelectric $\left(\Psi_{\mathrm{PE}}\right)$, ionizing $\left(\Psi_{\text {ion }}\right)$, Lyman-Werner $\left(\Psi_{\mathrm{LW}}\right)$, soft $(0.5-2 \mathrm{keV})$ and hard $(0.5-10 \mathrm{keV}) \mathrm{X}$-ray $\left(\Psi_{\mathrm{SX}}, \Psi_{\mathrm{HX}}\right)$, and IR bands (with $\Psi_{\mathrm{FIR}}=0$ again). The source terms for the non-trivial added bands are:

$\Psi_{\mathrm{PE}}= \begin{cases}271\left(1+t_{3.4}^{2}\right) & \left(t_{3.4} \leq 1\right) \\ 542 t_{3.4}^{-1.5} & \left(t_{3.4}>1\right)\end{cases}$
$\Psi_{\mathrm{LW}}= \begin{cases}109\left(1+t_{3.4}^{2}\right) & \left(t_{3.4} \leq 1\right) \\ 243 t_{3.4}^{-1.6} \exp \left(-t_{400}\right) & \left(t_{3.4}>1\right)\end{cases}$
$\Psi_{\mathrm{SX}}= \begin{cases}1.6 \times 10^{-6} & \left(t_{10} \leq 1\right) \\ 2.1 \times 10^{-6}+0.10 t_{10}^{-2} & \left(t_{10}>1\right)\end{cases}$
$\Psi_{\mathrm{HX}}= \begin{cases}1.6 \times 10^{-6} & \left(t_{10} \leq 1\right) \\ 1.6 \times 10^{-6}+0.15 t_{10}^{-2} & \left(t_{10}>1\right)\end{cases}$

where $t_{N} \equiv t /(N \mathrm{Myr})$. For the ionizing bands, we calculate $\Psi_{\text {ion, } v}$ in four sub-bands $v$ from 13.6-24.6, 24.6-54.4, 54.4-70, and 70$500 \mathrm{eV}$ in frequency, with $\Psi_{\text {ion, } v}=f_{v} \Psi_{\text {ion }}$ where $\Psi_{\text {ion }}$ is the total ionizing luminosity defined for our simpler default method above, and $f_{v}$ is the fraction in each band calculated assuming the emergent spectrum has a constant effective temperature set to $4 \times 10^{4} \mathrm{~K}$. The optical/NIR and NUV bands use the same $\Psi$ defined above for the 'default' network. All of the above are calculated from STARBURST99 average spectra, with the soft and hard X-ray bands empirically calibrated to observed X-ray binary populations.

The opacities in each band are given by $\left(\kappa_{\mathrm{NUV}}, \kappa_{\mathrm{opt}}, \kappa_{\mathrm{PE}}, \kappa_{\mathrm{LW}}\right.$, $\left.\kappa_{\mathrm{SX}}, \quad \kappa_{\mathrm{HX}}\right)=\left(1800 Z^{\prime}, 180 Z^{\prime}, 0.2+2000 Z^{\prime}, 2400 Z^{\prime}, 127+\right.$ $50 Z^{\prime}, 0.53+0.27 Z^{\prime}$ ) where $Z^{\prime}=Z / Z_{\odot}$. The $X$-ray cross opacities come from Thompson scattering and metal absorption following Morrison \& McCammon (1983) assuming solar abundance ratios. The LW cross-section accounts for dust shielding, but the self-shielding by molecular hydrogen is treated approximately as described below. The photoelectric opacity accounts approximately for molecular opacity at low temperatures and metallicities plus dust. For the FIR band, we calculate the opacity using the tables from Semenov et al. (2003) (specifically their Rosseland-mean opacities from their 'porous 5-layered sphere' dust models, as a bivariate function of the IR radiation temperature $T_{\mathrm{IR}}$ and dust temperature $\left.T_{\text {dust }}\right)$. For the ionizing bands, the cross-sections in each frequency range follow from the usual expressions for photoionizing absorption, scaling with the neutral $\mathrm{H}$ or neutral and partially ionized fractions for $\mathrm{He}$ (calculated as described in Paper I). Recall, these are used in our 'standard' scheme as well, the only difference is that we assume in the 'default' scheme that the ionizing photon spectrum always traces the UV background, while here each band is explicitly evolved independently.

The photoionization and photoelectric terms couple directly to the photoheating rates calculated in-code, as described in detail in Paper I. The NUV, optical/NIR, and FIR terms do not directly couple to the heating/cooling subroutines (except via the dust temperature, below). The Lyman Werner band is treated approximately as 
follows: in Paper I we describe in detail the cooling function used for the combination of molecular+metal-line (fine structure) cooling in primarily neutral $\left(\lesssim 10^{4} \mathrm{~K}\right)$ gas, which are fit to a table of CLOUDY simulations of gas slabs as a function of density, temperature, and metallicity. The terms presented in Paper I can be trivially divided into a term which vanishes as $Z \rightarrow 0$ (which represents the contribution from metal cooling) and one which remains constant (which is dominated by $\mathrm{H}_{2}$ cooling). In these simulations, we simply multiply the latter (metal-free) term by a function $f_{\mathrm{LW}}$ which ranges from 0 to 1 , determined by re-running the CLOUDY calculations for primordial $Z=0$ gas, illuminated by the given LW background. The soft and hard X-ray terms are included as Compton-heating (in addition to the other Compton heating/cooling terms described in Paper I), with heating rate per electron given by $\mathrm{d} W / \mathrm{d} t=u_{\gamma} \sigma_{\mathrm{T}}\left(\left\langle E_{\gamma}\right\rangle-4 k_{B} T\right) /\left(m_{e} c\right)$ where $u_{\gamma}$ is the photon energy density in the soft/hard band, $\sigma_{\mathrm{T}}$ the Thompson cross-section, $k_{\mathrm{B}}$ the Boltzmann constant, $m_{e}$ the electron mass, $c$ the speed of light, $T$ the gas temperature, and $E_{\gamma}$ the mean photon energy in the band (since the bands are narrow we simply take this to be the photon energy at the band median).
Finally, for the FIR band, we explicitly evolve the IR radiation field and dust temperature. We ignore PAH's and other very small grains where single-photon effects are important, and assume the dust-gas collision rate is lower than the dust-radiation absorption (a good assumption at densities $\lesssim 10^{6-10} \mathrm{~cm}^{-3}$ ), so the dust is simply taken to be in thermal equilibrium (and we assume geometric absorption here), with $T_{\text {dust }}^{4}=u_{\gamma} c / 4 \sigma_{B}$ where $u_{\gamma}$ is the local photon energy density integrated across all bands where dust dominates the opacity. The dust temperature $T_{\text {dust }}$ then influences the gas temperature via the dust-gas collisional heating term given in Paper I. The radiation field is updated at each time-step as an effective blackbody, assuming the dust emits radiation with radiation temperature equal to the dust temperature: if the dust re-emits a total energy $\Delta E_{\text {dust }}$ in one time-step into the zone, then $T_{\mathrm{IR}}^{4}(t+\Delta t)=$ $\left(E_{\gamma, \mathrm{IR}}(t) T_{\mathrm{IR}}^{4}(t)+\Delta E_{\text {dust }} T_{\text {dust }}^{4}\right) /\left(E_{\gamma, \mathrm{IR}}+\Delta E_{\text {dust }}\right)$. Likewise when radiation is exchanged between cells, the radiation temperature is updated accordingly.

This paper has been typeset from a $\mathrm{T}_{\mathrm{E}} \mathrm{X} / \mathrm{L} \mathrm{T} \mathrm{T}_{\mathrm{E}} \mathrm{X}$ file prepared by the author. 